Shuffler Instruments for the

Nondestructive Assay of Fissile

Materials

Phillip M. Rinard 


\section{CONTENTS}

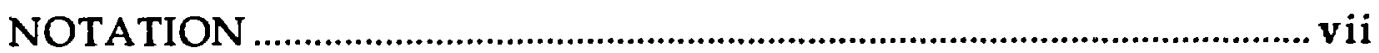

ABSTRACT

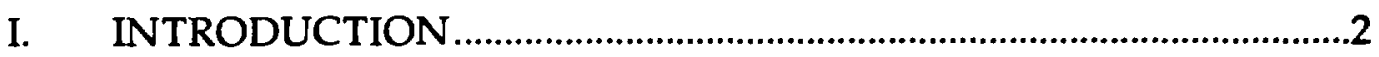

A. Shuffler Technique and Applications..............................................2

B. Delayed-Neutron Precursors ..............................................................4

C. Delayed-Neutron Production and Yields ..........................................5

D. Interfering Sources of Neutrons..........................................................

E. Shuffler Design Stages ..................................................................10

1. User Specifications ................................................................10

2. Conceptual Design and 252 Cf Sizing....................................10

3. Shielding .......................................................................................11

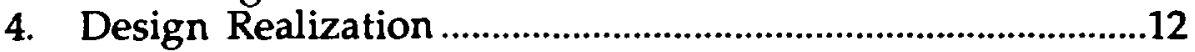

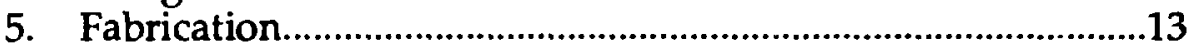

6. Testing and Calibration .......................................................13

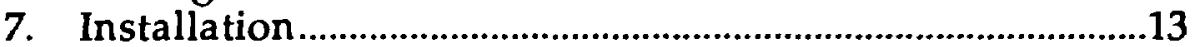

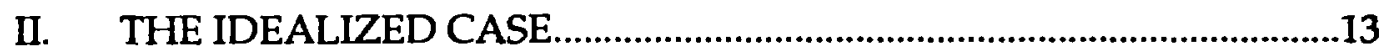

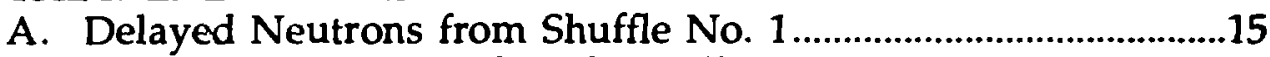

B. Delayed Neutrons in the $n$th Shuffle.............................................19

C. Delayed Neutrons from $n$ Shuffles..............................................21

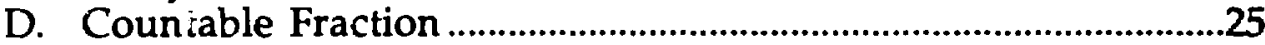

E. Maximizing Delayed-Neutron Counts .............................................26

III. IRREGULAR SHUFFLE TIMES AND NUMBERS ..........................27

A. Delayed-Neutron Counts with Irregular Times ...........................28

B. Adjusting Counts For Irregular Times .........................................30

C. Adjusting Counts for Numbers of Shuffles per Assay................31

IV. BACKGROUND, PRECISION, AND DETECTION LIMIT ................31

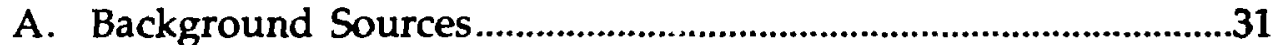

B. Counting Precision and Detection Limit.........................................31

C. Timing Optimization..............................................................................34

1. Maximum Delayed-Neutron Counts.....................................34

2. Minimum Relative Precision...................................................34

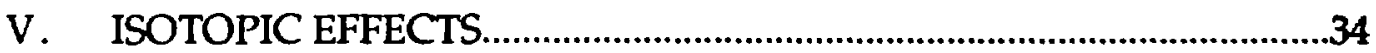

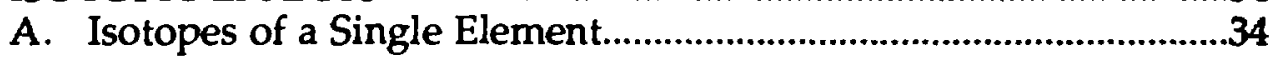

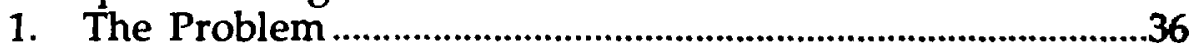

2. Detailed Isotopic Correction ...................................................38

3. Gross Isotopic Correction ........................................................38

B. Mixture of Uranium and Plutonium Isotopes................................39

VI. INHOMOGENEOUS MATERIALS ..................................................40

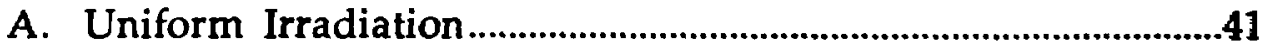

B. Detection Response Profile..............................................................42 


\section{CONTENTS (cont)}

VII. FLOWING LIQUID SHUFFLER

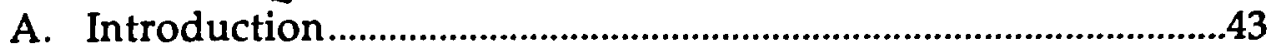

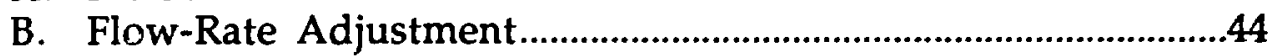

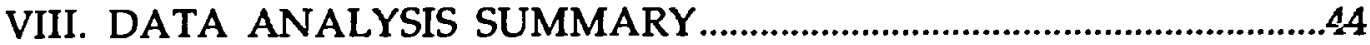

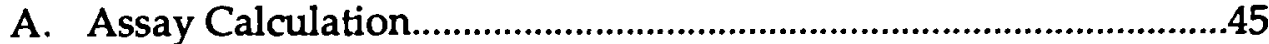

1. Start-up Calculations and Initializations ...............................46

2. Electronics Checks ...............................................................47

3. Intermediate Valies after a Shuffle ......................................47

4. Assay Count Rates.......................................................................48

5. Background and ${ }^{252} \mathrm{Cf}$ Decay Corrections...............................48

6. Flow-Rate Correction..............................................................49

7. Flux-Monitor Correction .....................................................49

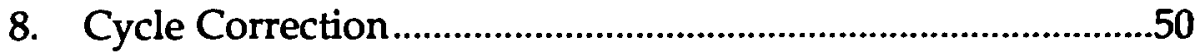

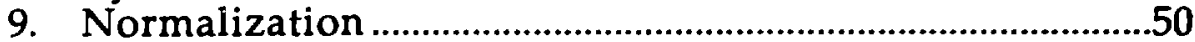

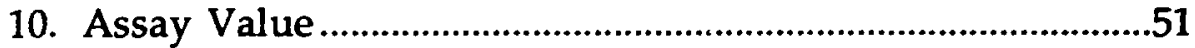

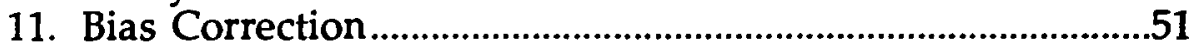

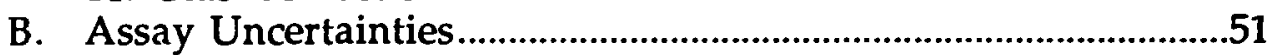

1. Random, Systematic, and Total............................................51

2. The Total Uncertainty ..............................................................52

3. Random and Systematic Uncertainties ...................................55

a. Delayed-Neutron Count Rate............................................55

b. Background Subtraction.........................................................56

c. Californium Decay …...............................................................56

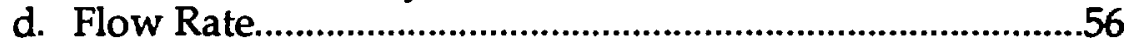

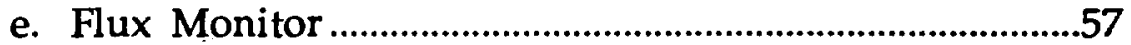

f. Cycle Ċorrection.................................................................57

g. Normalization .......................................................................58

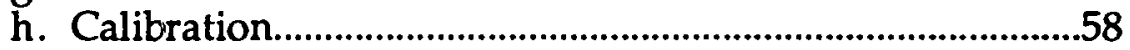

4. Total Uncertainty with Bias Correction......................................61

C. Generalized Numerical Techniques.............................................61

1. Interpolation from the Calibration Curve.............................61

2. Uncertainty in an Interpolated Value....................................61

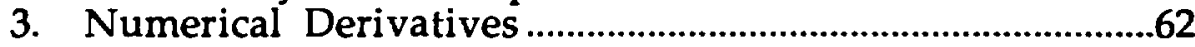

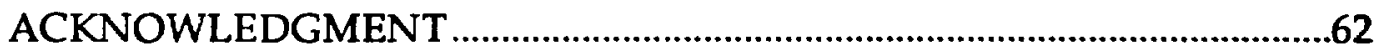

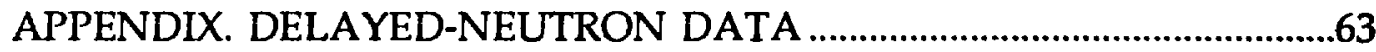

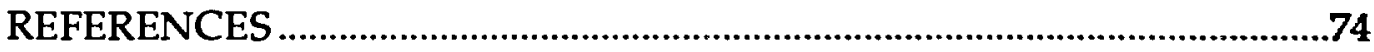




\section{NOTATION}

A $\quad=\quad$ atomic weight of fission target material

$A \quad=$ assay result $[\mathrm{g}, \mathrm{g} / \mathrm{L}]$

$A_{b c} \quad=$ bias corrected assay value

$A_{i} \quad=$ estimate of assay result

$A(i)=$ atomic weight of isotope $i$ in the fission target material

$A_{1} \quad=$ interpolated assay result

$a_{i}=$ coefficient for isotope $i$ for assay of a mixture of isotopes

$B=$ estimated background counts during an assay $=B_{b}\left(T_{d} / t_{b}\right)$

$B_{b} \quad=\quad$ background counts during a count time $t_{b}$

$b=$ background count rate from delayed-neutron detector $\left[\mathrm{s}^{-1}\right]$

$b_{i}=$ parameters in the bias correction function

$b_{M} \quad=$ background count rate from flux monitor $\left[\mathrm{s}^{-1}\right]$

$C=$ total counts (signal plus background)

$C_{D} \quad=\quad$ counts in the delayed-neutron detectors during an assay

$C_{M}=$ counts in the flux monitor detectors during an assay

$C_{D_{k}}=$ counts from the delayed-neutron detectors during shuffle $k$

$C_{M_{k}}=$ counts in the flux monitor detectors during shuffle $k$

$C F_{C f}={ }^{252} \mathrm{Cf}$ decay correction factor

$C F_{\text {cycle }}=$ cycle correction factor for irregular timings among shuffles

$C F_{\text {flow }}=$ flow rate correction factor

$C F_{f l u x}=$ flux monitor correction factor

$C F_{\text {norm }}=$ normalization factor

$c=$ total count rate (signal plus background) $\left[s^{-1}\right]$

$D=$ delayed-neutron counts from all groups for all $n$ shuffles' irradiations and counts that form an assay

$D_{\text {flow }}=$ delayed-neutron counts adjusted for flow rate

$D_{j}\left(s_{1}, s_{2}\right)=$ delayed-neutron counts from $j$ th group after irradiation in shuffle $s_{1}$ but counted only during shuffle $s_{2}$

$D_{j} \quad=\quad$ delayed-neutron counts from $j$ th group for all $n$ shuffles' irradiations and counts

$D_{j}(i)=$ delayed-neutron counts from the $j$ th group of isotope $i$ for all $n$ shuffles' irradiations and counts

$D_{j k} \quad=\quad$ term in the cycle correction factor for group $j$ and shuffle $k$

$D_{r}(i) \quad=\quad$ relative yield of delayed neutrons from isotope $i=f(i) \cdot Y(i)$ 

$D^{\prime} \quad=\quad D /(\varepsilon f)$ calculated using measured shuffle times
$D_{n}^{\prime} \quad=$ nominal value of $D^{\prime}$ calculated using nominal shuffle times
$D^{\prime \prime} \quad=$ counts measured with a flux monitor during an assay
$D_{n}^{\prime \prime} \quad=$ counts expected from a flux monitor during an assay (based on previous measurements)

$D(i)=$ delayed-neutron counts from isotope $i$ and from all groups for all $n$ shuffles' irradiations and counts that form an assay

$D($ totel $)=$ delayed-neutron counts from all isotopes and from all groups for all $n$ shuffles' irradiations and counts that form an assay

$d=$ delayed-neutron count rate

$d_{1} \quad=$ delayed-neutron count rate corrected for background and ${ }^{252} \mathrm{Cf}$ decay

$d_{2}=$ delayed-neutron count rate corrected for background, $252 \mathrm{Cf}$ decay, and flow rate

$d_{3}=$ delayed-neutron count rate corrected for background, $252 \mathrm{Cf}$ decay, flow rate, and flux monitor

$d_{4}=$ delayed-neutron count rate corrected for background, $252 \mathrm{Cf}$ decay, flow rate, flux monitor, and cycle irregularities

$d_{5}=$ delayed-neutron count rate corrected for background, $252 \mathrm{Cf}$ decay,

flow rate, flux monitor, cycle irregularities, and normalization

$d d=$ number of days of decay of the ${ }^{252} \mathrm{Cf}$

$F \quad=$ liquid flow rate $[\mathrm{L} / \mathrm{h}]$

$F_{\text {std }}=$ standard flow rate $[\mathrm{L} / \mathrm{h}]$

$f \quad=$ fission neutron production rate in assay sample $=m m N_{A} \sigma_{f} / A$

$f(i)=$ fission neutron production rate by isotope $i$ in the assay sample $=$ $v(i) m(i) N_{A} \sigma_{f}(i) \varphi / A(i)$

$f\left(x_{s}, x_{m}\right)=$ fission neutron production rate as function of source and material positions [ $\left.\mathrm{s}^{-1}\right]$

$f_{\text {bias }}=$ bias correction function

$f_{\text {calib }}=$ calibration function

$g_{i}=$ parameters in flow rate correction function

$I(i)=$ fraction by mass of isotope $i$ in the target material

$i \quad=\quad$ isotope index

$j=$ index of precursor groups $=1,2, \ldots, 6$

$k=$ index of shuffles within an assay $=1,2, \ldots, n$ 


\section{NOTATION (cont)}

$L=$ active length of the detector tubes [cm]

$m \quad=\quad$ mass of fission target material [g]

$m \quad=\quad$ uncorrected flux monitor count rate after $n$ shuffles

$m_{C f} \quad=$ mass of $252 \mathrm{Cf}$

$m_{1}=$ reference flux monitor count rate, corrected for background and ${ }^{252} \mathrm{Cf}$ decay

$m_{n} \quad=\quad$ nominal value of $m_{1}$

$m(i)=$ mass of isotope $i$ of the fission target material

$N_{A}=$ Avogadro's number $=6.022169 \times 10^{23}[$ atoms $/ \mathrm{mole}]$ or [atoms/( $A$ grams)]

$N F \quad=$ normalization factor

$n=$ number of shuffles in an assay

$n_{n} \quad=$ nominal number of shuffles in an assay

$P_{j} \quad=\quad$ population of the $j$ th precursor group

$P^{\prime}{ }_{j s}=$ precursor population from all previous shuffles at the end of the $(s-1)$ counting interval

$=$ cycle correction factor related to precursor population

$=$ parameters in the calibration function

$=\quad$ value carried between shuffles for the cycle correction factor

$=\exp \left(-\lambda_{j} \tau\right)$

$=$ parameters in flux monitor correction function

$=$ radius of ring of detectors $[\mathrm{cm}]$

$=$ index of successive shuffles $=1,2, \ldots, n$

$=$ total time spent on background and assay [s]

$=$ time $[\mathrm{s}]$

$=$ background count time $[\mathrm{s}]$

$=\quad$ sum of all shuffle count times during an assay $[s]=n t_{c}$

$=$ total irradiation time; the sum of the $t_{i k}$ in an assay

$=$ nominal count time

$=$ count time $[\mathrm{s}]$

$=$ time of counting delayed neutrons during shuffle $s$

$=$ time to move the californium source "forward" into the irradiation position [s]

$t_{f n} \quad=$ nominal forward travel time 
$t_{f s}=$ time of forward travel during shuffle $s$

$t_{i}=$ irradiation time [s]

$t_{\text {in }}=$ nominal irradiation time

$t_{\text {is }}=$ time of irradiation during shuffle $s$

$t_{r} \quad=$ time to move the californium source in "reverse" to the storage position [s]

$t_{r n} \quad=$ nominal reverse travel time

$t_{r s} \quad=\quad$ time of reverse travel during shuffle $s$

$x_{m}=$ coordinate of material segment being irradiated

$x_{s}(t)=$ coordinate of neutron source during a scan

$Y(i)=$ absolute delayed-neutron yield per fission of isotope $i$

$$
=\quad v(i) \sum_{j} \beta_{j}(i)
$$

$z \quad=\quad$ distance from bottom of detector tube $[\mathrm{cm}]$

$\beta_{j}=$ fractional delayed-neutron yield from $j$ th group (a fraction of the average number of neutrons emitted per fission)

$\beta_{j}(i)=$ fractional delayed-neutron yield from $j$ th group for isotope $i$

$\delta=$ deadtime correction coefficient [s]

$\varepsilon=$ detection efficiency

$\lambda=$ decay constant of ${ }^{252} \mathrm{Cf}=0.0007172 \mathrm{~d}^{-1}$ (half-life $=2.646 \mathrm{yr}$ )

$\lambda_{j} \quad=$ decay constant of $j$ th group $\left[\mathrm{s}^{-1}\right]$

$v \quad=\quad$ average number of neutrons emitted per fission

$v(i)=$ average number of neutrons emitted per fission by isotope $i$

$\sigma_{B}=$ precision of a background count $B$

$\sigma_{C} \quad=\quad$ precision of a total count $C$

$\sigma_{D} \quad=$ precision of a delayed-neutron count $D$

$\sigma_{f} \quad=$ spectrum-weighted microscopic fission cross section $\left[\mathrm{cm}^{2}\right]$

$\sigma_{f}(i)=$ spectrum-weighted microscopic fission cross section of isotope $i\left[\mathrm{~cm}^{2}\right]$

$\sigma_{x}$ or $\sigma(x)=$ uncertainty in the quantity $x$

$\sigma_{x}^{2}=$ variance in the quantity $x$

$\sigma_{x y}=$ covariance between the quantities $x$ and $y$

$\sigma_{r}=$ random uncertainty in the assay value

$\sigma_{s} \quad=\quad$ systematic uncertainty in the assay value

$\sigma_{A} \quad=\quad$ total uncertainty in the assay value 


\section{NOTATION (cont)}

$\tau=$ shuffle time (or period, or cycle time) $=t_{f}+t_{i}+t_{r}+t_{c}[\mathrm{~s}]$

$\tau_{n} \quad=$ nominal shuffle time in the parameter file

$\tau^{\prime} \quad=\tau-\left(t_{f}+t_{r}\right)[s]$

$\varphi \quad=\quad$ irradiation neutron flux $\left[\mathrm{n} / \mathrm{cm}^{2} \cdot \mathrm{s}\right]$

$\Omega \quad=\quad$ solid angle subtended by detector ring 


\title{
SHUFFLER INSTRUMENTS \\ FOR THE NONDESTRUCTIVE ASSAY OF FISSILE MATERIALS
}

\author{
by
}

Phillip M. Rinard

\begin{abstract}
A shuffler is a nondestructive assay instrument used to determine the fissile content of materials. It places an isotopic source of neutrons near the material to induce fissions, withdraws the source, and counts the delayed neutrons. The source is shuffled until a sufficient number of delayed neutrons have been counted.

The shuffler technique is generally applied to difficult assay cases. The amount of material present may be very small (a few milligrams), and thus it does not spontaneously emit neutrons of consequence; the amount of material is also below an active well counter's level of sensitivity. On the other hand, the fissile amount may be fairly large, but the rate of spontaneously emitted neutrons may still be low (so a passive neutron count will not work) or the highest assay precision may be desired (favoring a shuffler over an active well counter) even if the material is inhomogeneous (making it difficult to interrogate with thermal neutrons). In all these cases, gamma-ray backgrounds, self-shielding, or matrix effects can make gammaray assays impractical.

Materials ranging from highly radioactive spent-fuel assemblies to lowlevel waste drums have been assayed with shufflers, as have leached hulls, various process materials, scrap, and waste.

This report presents a theoretical background for shufflers and describes techniques for practical applications. Procedures for assaying mixtures of fissile isotopes, inhomogeneous materials, and flowing liquids are discussed. It is shown how the precision and limits of detection of a shuffler can be calculated for a given neutron background rate. A section on data analysis gives a stepwise procedure for converting the measured counts into an assay value, including random, systematic, and total uncertainties.
\end{abstract}




\section{INTRODUCTION}

\section{A. Shuffler Technique and Applications}

A shuffier is a nondestructive assay (NDA) instrument to induce fissions and measure delayed neutrons for the purpose of performing an NDA of fissile materials. The delayed neutrons are emitted by fission products (precursors) as they decay. The time delay between fissions and neutron emissions can be a few minutes, but more often is less than a minute.

Shufflers are generally employed for the NDA of fissile materials in situations where it is difficult to assay by other means. The material may emit only smali amounts of neutrons or gamma rays because of low intrinsic emission rates or because only small amounts of materials are involved; the gamma-ray attenuation may be too large or too poorly known to perform a gamma-ray assay accurately; intense gamma rays from fission products and passive neutron emissions from transuranic isotopes may prevent assays with passive gamma rays or neutrons.

Delayed neutrons can still be counted in these difficult assay cases because

(1) they can be produced in sufficient quantities from small amounts of materials in reasonable assay times,

(2) they are more penetrating and less susceptible to attenuation by the matrix than gamma rays,

(3) they can be measured in the presence ot intense passive gamma rays because the neutron detectors can be protected from the gamma rays by thick shields without degrading the neutron signal significantly, and

(4) the delayed-neutron signal can be measured in the presence of passive neutrons (although the counting precision suffers as the passive neutron intensity increases).

A few tens or hundreds of micrograms of ${ }^{252} \mathrm{Cf}$ are used as a source of irradiating neutrons because of ${ }^{252} \mathrm{Cf}$ 's high intrinsic emission rate $\left(2.34 \times 10^{6} \mathrm{n} / \mathrm{s} / \mu \mathrm{g}\right)$ and small size. The half-life of ${ }^{252} \mathrm{Cf}$ is $2.65 \mathrm{yr}$, so a new source is needed every 3 to $5 \mathrm{yr}$.

Other active neutron assay techniques have strengths and weaknesses relative to shufflers. Active well counters are much more portable, but the irradiating sources are always near the assay chamber where they create a large background and degrade the precision and detection limit. Small accelerators using the $(d, t)$ reaction do not generate neutrons during the delayed-neutron count time, but cannot produce an irradiating flux of high-energy neutrons as large as even a small ${ }^{252} \mathrm{Cf}$ source; the detection limit is thu:; again likely to be higher than a shuffler's. Differential die-away systems use $(d, t)$ generators and assay with thermal neutrons to increase the sensitivity to fissile materials while giving up the penetrability of large, dense materials by high-energy neutrons. Photoneutron sources have been used in a Los Alamos welllogging instrument and the Los Alamos Rover nuclear rocket program; a very intense 
gamma-ray source such as ${ }^{124} \mathrm{Sb}$ is needed, but the half-life is inconveniently short (60 days) and the irradiating flux is again less than from a relatively small ${ }^{252} \mathrm{Cf}$ source.

References 1-3 contain additional general information about delayed-neutron assay systems and techniques. Los Alamos has built shufflers for process materials (cans of $\mathrm{UO}_{2}, \mathrm{UF}_{6}$, mixed oxide, and uranium ore), wastes and scrap (sweepings, solutions, leached hulls, and alumina), products [billets, processed highly enriched uranium (HEU)], and HEU spent-fuel assemblies. Shufflers of three different sizes are shown in Figs. 1-3. Containers range from small cans to large drums, whereas fissile

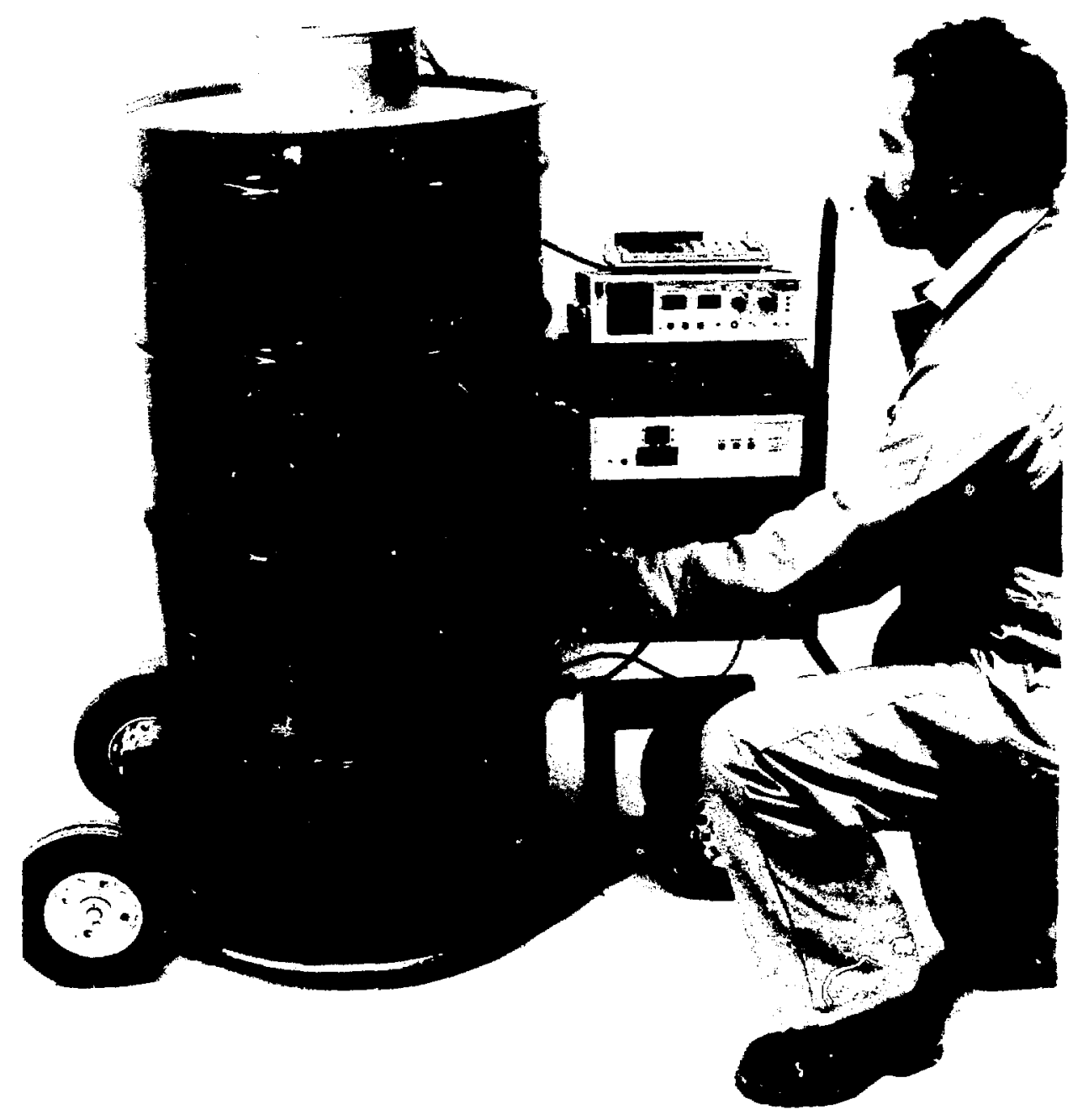

Fig. 1. A small shuffler was built to assay the ${ }^{235} \mathrm{U}$ content in $10-\mathrm{g}$ samples of $\mathrm{UF}_{6}$. The sensitivity was $4 \mathrm{mg}{ }^{235} \mathrm{U}$ ( $3 \sigma$ above background) with a 1000 -s count. The small ${ }^{252} \mathrm{C}$ s source yielded $8 \times 10^{6} \mathrm{n} / \mathrm{s}$. The background count rate was 12 counts $/ \mathrm{s}$. The nonuniform distribution of $\mathrm{UF}_{6}$ in the samples made gamma-ray assays impractical. 


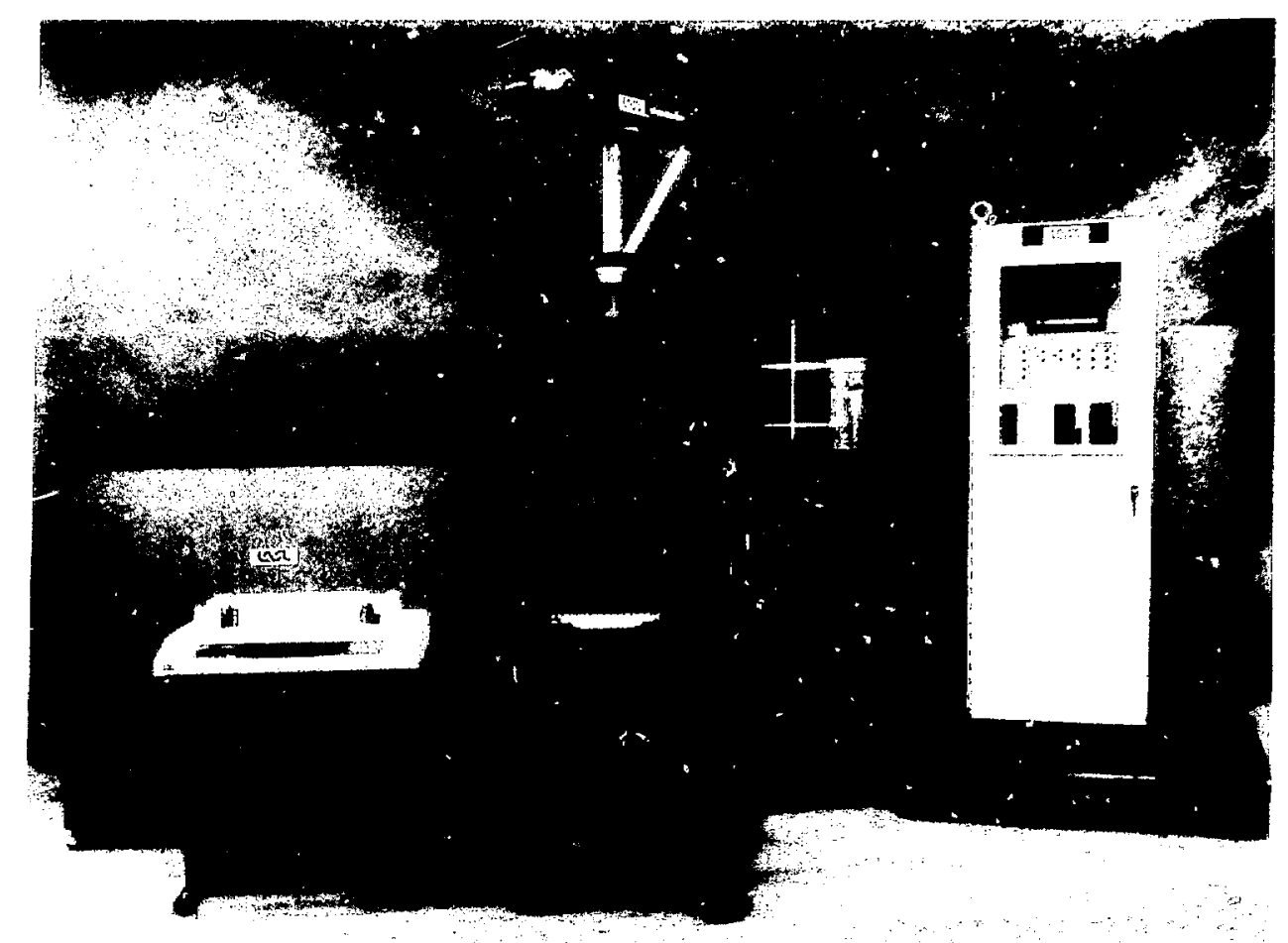

Fig. 2. This medium-sized shuffler assays scrap and waste items, such as highpurity uranium-aluminum alloy ingots, castings, saw and lathe chips, low-purity oxides of uranium mixed with flux materials, floor sweepings, and residues from scrap recovery operations. The body of the shuffler is behind a printer and computer terminal; a hoist assists with loading and unloading heavy objects. Most of the shuffler is shiclding to protect personnel from the ${ }^{252} \mathrm{Cf}$ source's radiations. The electronics are in the rack to the right. Source strengths may be as high as $6 \times 10^{8} \mathrm{n} / \mathrm{s}$.

masses can range from milligrams to kilograms. More details of some of the Los Alamos shufflers are given in Ref. 4.

\section{B. Delayed-Neutron Precursors}

As a nucleus fissions into two daughter nuclei (or fission products), some neutrons are simultaneously emitted; these are "prompt" neutrons. Many of the daughter nuclei have an excess of neutrons compared to the stable isotopes and undergo negative beta decays. The nuclei created by beta decays may be in excited states. The normal result is gamma-ray emission, but it is also possible to release neutrons instead. These are "delayed" neutrons because their emissions have the half-lives of the beta decays and are released well after the fission event. A fission product that undergoes beta decay followed by the emission of a neutron is a delayed-neutron precursor.

Although a large number of fission products will emit delayed neutrons, 3,5,6-8 their combined activities are accurately represented by a sum of six simple exponential decays, 3,5 each representing a group of isotopes. 


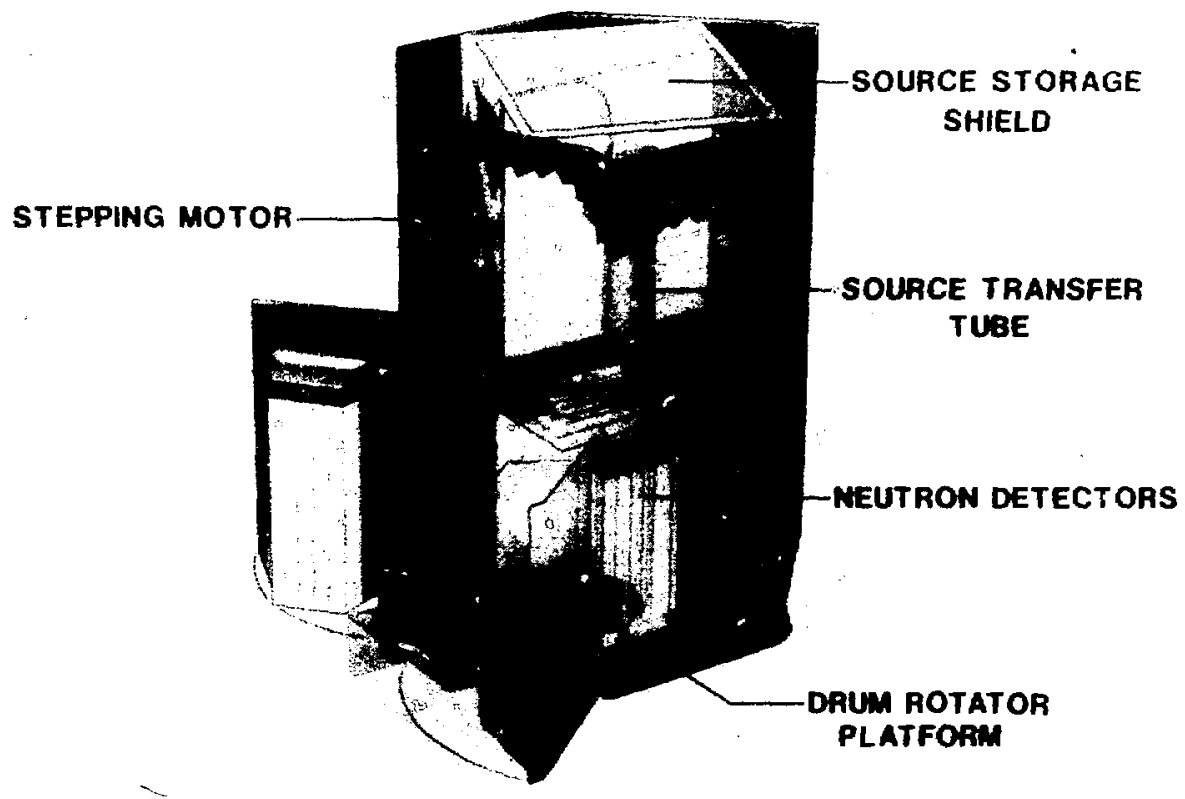

Fig. 3. Large shufflers are needed for assaying objects like 55-gal. drums. A drum with waste may have assorted matrix materials. Often the objective is to verify that the amount of fissile material in the drum meets the requirements for disposal. This shuffler has a large ${ }^{252} \mathrm{Cf}$ source $\left(1 \times 10^{9} \mathrm{n} / \mathrm{s}\right)$ stored above the assay chamber; the source can also be stored below the shuffler if there is more room in that direction. Other shufflers of this size have front and rear doors with the exit path chosen according to the result of the assay.

A few of the likely group members (precursors of delayed neutrons), following fission of $235 \mathrm{U}$ by thermal neutrons, are shown in Table I. Only individual precursors that contribute at least $1 \%$ of the total delayed neutrons are included; over 200 more precursors not included in Table I are given in Refs. 7 and 8, but these account for only $13 \%$ of all the delayed neutrons. Some of the precursors clearly belong to certain groups (based on the half-lives), while other assignments are not as certain.

\section{Delayed-Neutron Production and Yields}

A source of neutrons is needed to induce fissions in the material, and then it must be turned off or removed to prevent interference with the counting of the delayed neutrons. The energy spectrum of the source neutrons and the spectrum-modifyirig isotopes in the material being assayed affect the delayed-neutron yields of the precursors.

An isotopic source of neutrons (generally $252 \mathrm{Cf}$ ) is positioned near the material for a few seconds and removed; the delayed neutrons are then counted. The less time it takes to move the source, the more effective the shuffling, although transfer times as long as $1.5 \mathrm{~s}$ have worked quite well. With small materials (for example, fuel pellets, flowing liquids) it may be more convenient to leave the irradiating source stationary 
TABLE I. Yields of Precursors for Delayed Neutrons After Thermal Fission of ${ }^{235} \mathrm{U}^{7}$

Only precursors contributing at least $1 \%$ of all delayed neutrons (DN) are given here. Group assignments are partially from Keepin. 5

\begin{tabular}{|c|c|c|c|}
\hline Group & Nuclide & $\begin{array}{c}\text { Half-Life } \\
\text { (s) }\end{array}$ & $\%$ of DN \\
\hline 1 & 35 Br 87 & $\begin{array}{l}55.70 \\
55.70\end{array}$ & $\frac{3.33}{3.33}$ \\
\hline 2 & $\begin{array}{l}53 \text { I } 137 \\
35 \mathrm{Br} 88\end{array}$ & $\begin{array}{l}24.50 \\
16.00 \\
22.72\end{array}$ & $\begin{array}{r}13.9 \\
7.21 \\
21.11\end{array}$ \\
\hline 3 & $\begin{array}{l}53 \mathrm{I} 138 \\
37 \mathrm{Rb} 93 \\
35 \mathrm{Br} 89\end{array}$ & $\begin{array}{l}6.50 \\
5.86 \\
4.38 \\
6.22\end{array}$ & $\begin{array}{r}5.28 \\
2.80 \\
10.7 \\
18.78\end{array}$ \\
\hline 4 & $\begin{array}{l}53 \text { I } 139 \\
35 \text { Br } 94 \\
33 \text { As } 85 \\
51 \text { Sb } 135 \\
35 \text { Br } 90 \\
55 \text { Cs } 143 \\
39 \text { Y } 99\end{array}$ & $\begin{array}{l}2.38 \\
2.76 \\
2.03 \\
1.82 \\
1.80 \\
1.78 \\
1.40 \\
2.30\end{array}$ & \begin{tabular}{r|}
3.61 \\
9.84 \\
6.27 \\
1.53 \\
7.94 \\
1.48 \\
2.51 \\
33.18
\end{tabular} \\
\hline 5 & $\begin{array}{l}35 \mathrm{Br} 91 \\
9 \mathrm{Y} 98 \mathrm{M} \\
37 \mathrm{Rb} 95\end{array}$ & $\begin{array}{l}0.60 \\
0.65 \\
0.38 \\
0.61\end{array}$ & $\begin{array}{l}2.09 \\
3.21 \\
\underline{3.63} \\
8.93\end{array}$ \\
\hline 6 & $\begin{array}{l}37 \mathrm{Rb} 96 \\
33 \text { As } 87\end{array}$ & $\begin{array}{l}0.20 \\
0.30 \\
0.23\end{array}$ & $\begin{array}{l}1.20 \\
\frac{1.16}{2.36}\end{array}$ \\
\hline & & & 87.69 \\
\hline
\end{tabular}


and move the material. ${ }^{9}$ In either case, something is being shuffled back and forth while delayed neutrons are being generated and counted.

The average number of neutrons (including delayed neutrons) emitted by a fission is $v$. A fraction $\beta_{j}$ of $v$ appears in group $j$ of the delayed neutrons with a decay constant of $\lambda_{j}$. The absolute yield (the number of delayed neutrons per fission) of the $j t h$ precursor is thus $\beta_{j} v$. The yield rate (the number of delayed neutrons per second per fission) is a function of time; for the $j$ th precursor, the yield rate after a fission is $\lambda_{j} \beta_{j} v \exp \left(-\lambda_{j} t\right)$. The yield rates for thermal fission of $235 \mathrm{U}$ are shown in Fig. 4 for all six precursors and their sum, as functions of the decay time after fission. Much more detailed information for many important isotopes is given in the Appendix.

The total delayed-neutron yields $\left(\Sigma \beta_{j} v\right)$ of important isotopes are shown in Fig. 5. The yield from a given element increases with atomic weight because the number of "excess" neutrons after fission becomes greater.

The yields from individual uranium and plutonium precursor groups $\left(\beta_{j} v\right)$ are compared in Figs. 6 and 7. In six of these eight isotopes, group 4 is the dominant group, but in two of the plutonium isotopes group 5 dominates.

The fission cross sections, delayed-neutron yields, and decay constants are the basic information for shuffler assays, but fissions in a shuffler are induced over several seconds (not in an instantaneous burst), by an irradiating spectrum modified by the material being assayed. Not all delayed neutrons are counted (which would require several minutes between irradiations). The scheme given in Sec. II shows that the count precision can be optimized by selecting appropriate irradiation and count times.

\section{Interfering Sources of Neutrons}

During the counting of delayed neutrons, other undesirable neutrons can be generated by several sources:

- Passive emission from the material being assayed or the shuffler's environment (these must be subtracted from the delayed count);

- Neutrons from the ${ }^{252} \mathrm{Cf}$ source may reach the detectors even though the source is separated from the detectors by shielding material;

- Induced fissions in uranium and plutonium isotopes from gamma rays from fissirn products (Fortunately, the photofission cross sections and yields per fission are negligibly small from uranium and plutonium even with gamma-ray energies of several MeV.); 10

- $(n, p)$ reactions in ${ }^{17} \mathrm{O}$ that can lead to delayed neutrons from the decay of ${ }^{17} \mathrm{~N}$ with a half-life of $4.17 \mathrm{~s}^{11-13}$ (Although this has been observed in reactor cooling water, the cross section and delayed-neutron yield are too small to be detected with the lower irradiating neutron fluxes in a shuffler.); and

- $(n, d)$ reactions with 180 that can also result in delayed neutrons from the decay of ${ }^{17} \mathrm{~N} .11$ (The yield from this reaction is again negligible in a shuffler.) 


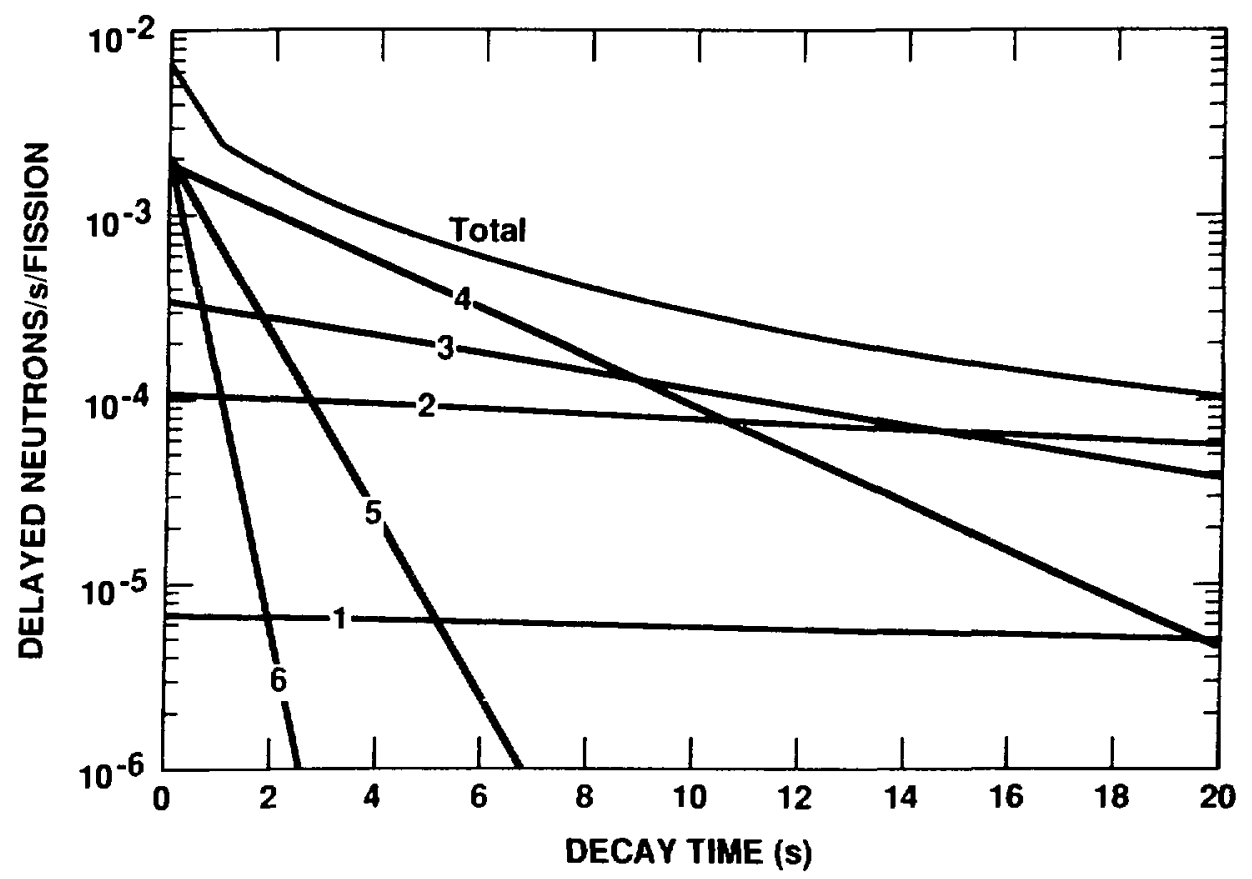

Fig. 4. The emission rates of delayed neutrons from the six precursor groups are shown following a fission of ${ }^{235} \mathrm{U}$ by a thermal neutron. Each precursor's emission rate decays exponentially.

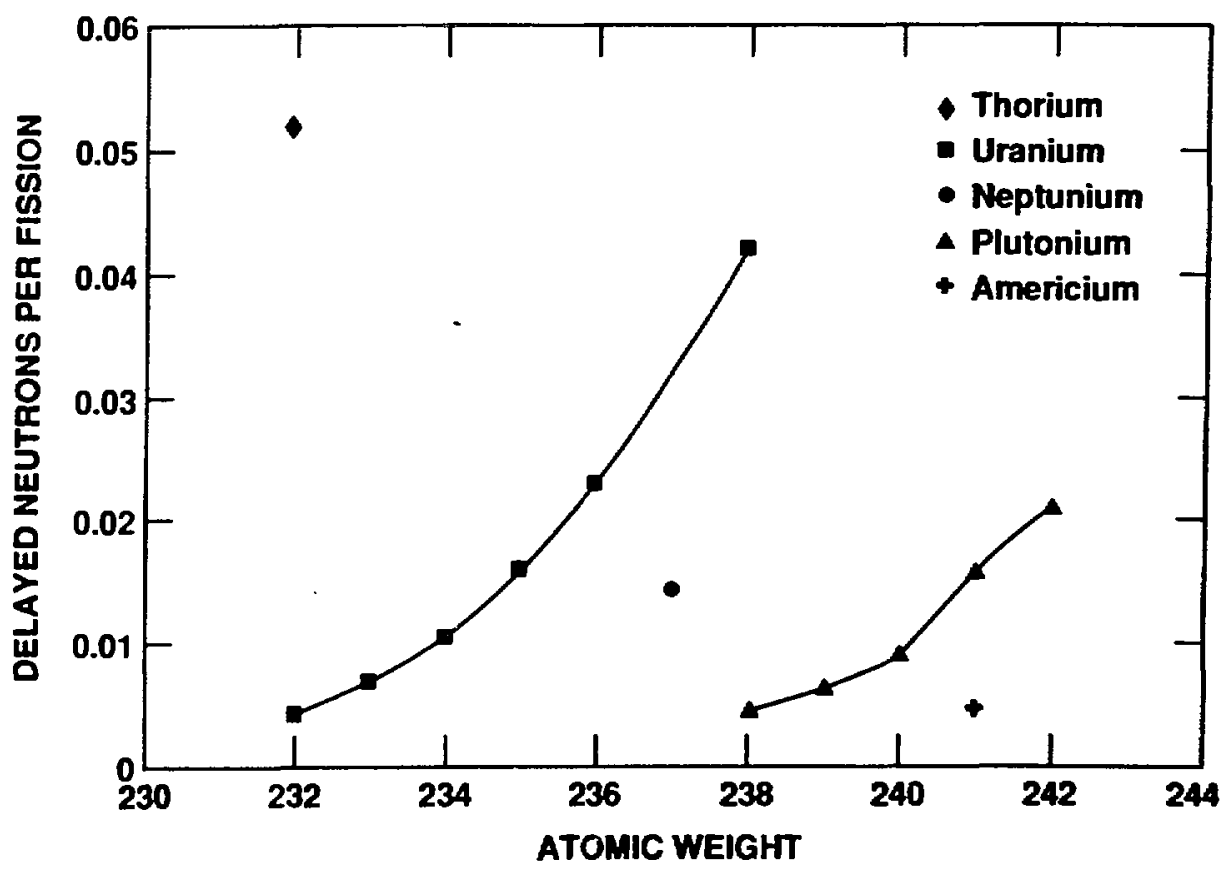

Fig. 5. The number of delayed neutrons produced in each fission depends on the fissioning isotope. The isotopes of uranium and plutonium are connected by lines to show that more delayed neutrons are produced when the number of neutrons in the nucleus is large. 


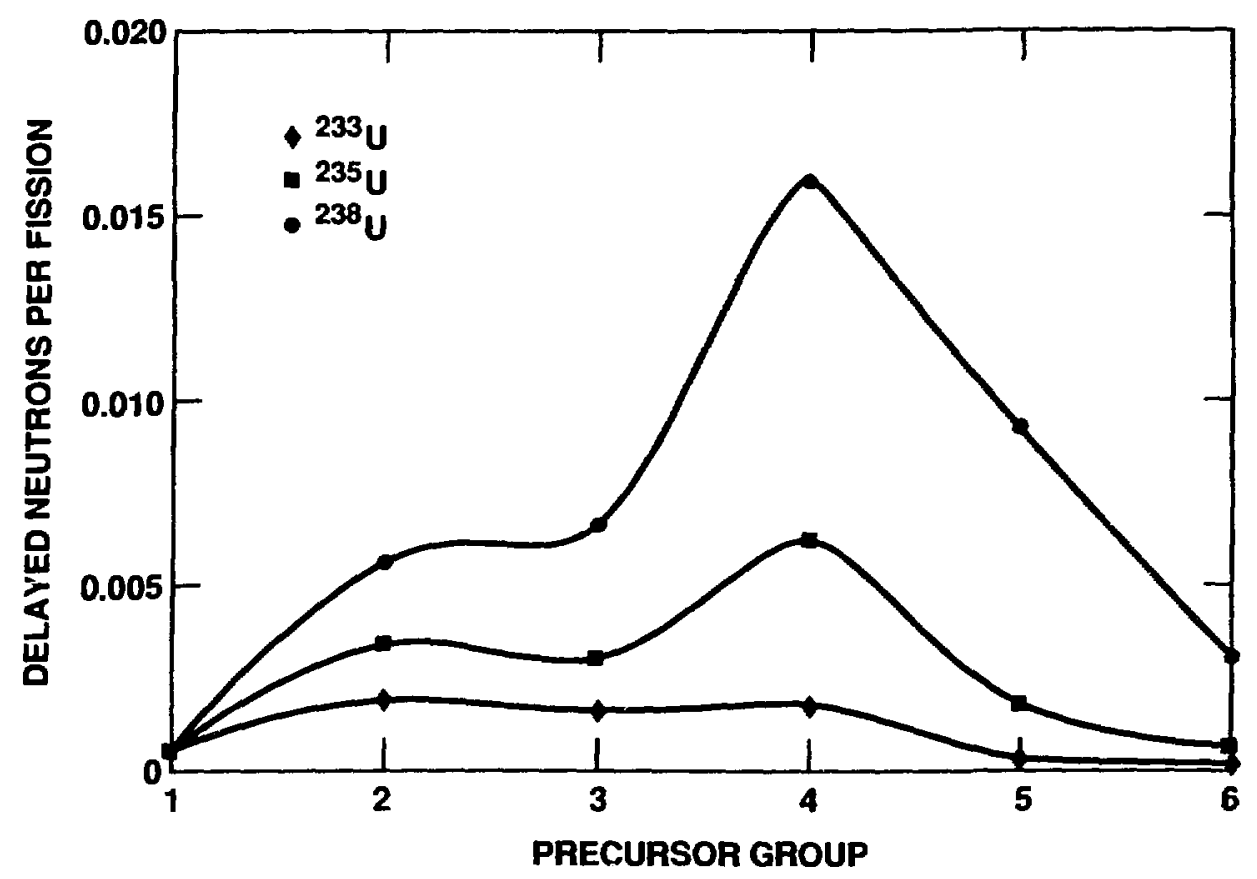

Fig. 6. The delayed-neutron yields from three uranium isotopes are compared for the six precursor groups. The importance of group 4 grows as the atomic weight increases. These data are taken from the Appendix.

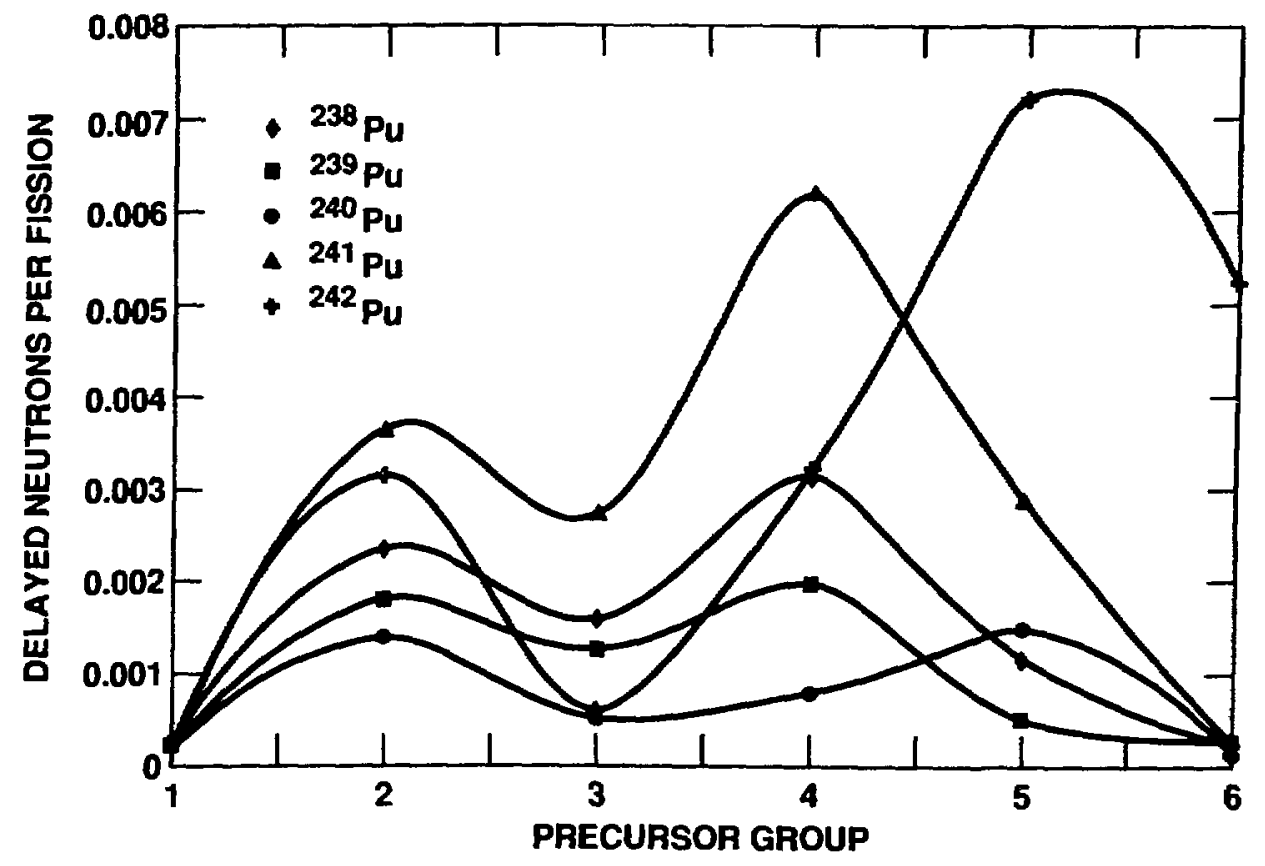

Fig. 7. The delayed-neutron yields from five plutonium isotopes are compared for the six precursor groups. Group 4 is less important than group 5 for the lightest and heaviest isotopes. Group 6 is unusually important for ${ }^{242} \mathrm{Pu}$. These data are taken from the Appendix. 
Multiplication, photofission, and ( $n, p)$ reactions will iot be specifically considered further in this report. Background effects in general, regardless of the origins, are treated in detail later.

\section{E. Shuffler Design Stages}

1. User Specifications. A shuffler design begins with the user specifications. What materials in what geometries are to be measured? What are the ranges of masses and isotopes? What assay precision and accuracy are desired? How much time is available to complete an assay? In what neutron and gamma-ray backgrounds must the measurements be made? How many years of use are needed for the $252 \mathrm{Cf}$ source? What are the maximum permissible radiation dose rates to personnel? How does the shuffler interact with the site (human interface, electrical signals)?

2. Conceptual Design and ${ }^{252} \mathrm{Cf}$ Sizing. A conceptual design of the assay region and the corresponding size of the ${ }^{252} \mathrm{Cf}$ source are estimated from the information in the user specifications and from the calculations described later. It is impossible to separate the design and the ${ }^{252} \mathrm{Cf}$ size, because the measurement precision depends on the geometry of the assay region as well as the size of the ${ }^{252} \mathrm{Cf}$ source. For example, if a small number of detertor tubes is used, the source will have to be relatively large.

The assay chamber must have a cavity large enough to hold the objects to be assayed. A ring of ${ }^{3} \mathrm{He}$ detectors is normally placed around this chamber with the detectors embedded in polyethylene; for large objects, detectors are also placed above and below the object. The center of a 1-in.-diam detector is typically about an inch and a half inside the polyethylene and the center-to-center spacing between detectors is about 2 in. These rules of thumb provide a starting point for Monte Carlo calculations with trial geometries. For example, if the material to be assayed is also a good moderator, the detectors may be more effective if placed closer than an inchi and a half from the surface of the polyethylene.

The length of the detector tubes can be estimated from geometric considerations only. Details are given in Sec. VI.B.

Monte Carlo calculations can give the fission rates per source neutron, the delayed-neutron count rate, and the detection efficiencies for different geometries. The effects of reflectors, moderators, and poisons can also be examined. Heavy metal reflectors "behind" the ${ }^{252} \mathrm{Cf}$ source have been used to increase the neutron flux in the material being assayed. A thin layer of cadmium may encase the assay region to prevent the return of thermal neutrons into it from the polyethylene; thermal neutrons may interact with only the skin of the fissile material, producing a nonuniform irradiation and a misleading response.

After the assay region has been optimized, the fission rate (possibly in different regions of the material), delayed-neutron count rate, and detection efficiency are known. But a further calculation is needed to estimate the countable fraction of delayed neutrons. Only a portion of the delayed neutrons produced have a chance of being counted because the detectors are not turned on until the ${ }^{252} \mathrm{Cf}$ source has been returned to its storage position (taking about a second) and are left on for only a few 
seconds (approximately 10 ) before the ${ }^{252} \mathrm{Cf}$ source is returned for another irradiation. The mathematics of the countable fraction are given in Sec. II.D.

At this point, the minimum size of the ${ }^{252} \mathrm{Cf}$ needed to meet the required relative precision can be estimated. The process described here makes use of the information developed in this report; other procedures are either equivalent or more suitable for the circumstances at hand.

Section IV.B shows how the pertinent assay parameters are related. A search over the unknown parameters can be done to find the set of values that minimize the mass of ${ }^{252} \mathrm{Cf}$. The basic steps in finding the ${ }^{252} \mathrm{Cf}$ size are these:

- The relative precision $\sigma_{D} / D$ is specified $b y$ the user.

- The number of delayed-neutron counts is

$m_{252} \cdot\left(2.34 \times 10^{6} \mathrm{n} / \mathrm{s} / \mu \mathrm{g}{ }^{252} \mathrm{Cf}\right) \cdot$ (number of delayed-neutron counts per where $m_{C f}$ is the mass of ${ }^{252} \mathrm{Cf}$ in micrograms. The third factor is found from Monte Carlo calculations and the countable fraction.

- The background rate must be estimated; this can include neutrons frum spontaneous fissions from the material (for example, ${ }^{238} \mathrm{U}$ ), from $(\alpha, \mathrm{n})$ reactions, from the ${ }^{252} \mathrm{Cf}$ source, and from nearby materials (either directly or through cosmic-ray interactions).

- The total time allowed for one assay is specified by the user. It is useful, but not necessary, to estimate the relative durations of the irradiation and count times during a shuffle.

- The relation for $\sigma_{D} / D$ (Sec. IV.B) can then be evaluated at different sets of the remaining parameters $\left(m_{C f}\right.$, background count time, and possibly the total delayed-neutron count time). The smallest $m_{C f}$ that will meet the measurement requirements has the advantage of minimizing the volume of shielding.

To be useful for three years, the size just found should be multiplied by 2.2. Depending on the faith placed in all of these calculations, the ${ }^{252} \mathrm{Cf}$ size can be increased further to allow for underestimates. For example, the geometry used in the Monte Carlo calculation may not be in complete agreement with the geometry of the as-built instrument, so an additional factor of 2 is prudent.

3. Shielding. Shielding is needed to protect personnel and to isolate the ${ }^{252} \mathrm{Cf}$ source (and any other radiation sources). The materials and thicknesses must be chosen to meet the site-specific requirements. Reference 14 is a practical guide for a few material types.

If a hydrogenous material (such as polyethylene) alone is used, the attenuation of ${ }^{252} \mathrm{Cf}$ primary gamma rays is poor and the production of high-energy gamma rays 
following neutron capture in hydrogen is a serious problem. A small shield of lead or tungsten immediately around the ${ }^{252} \mathrm{Cf}$ greatly helps with the primary gamma rays, but for large shields, an inch or more of lead is still needed on the outside of the polyethylene to attenuate the capture gamma rays. A rule of thumb for neutron-doserate attenuation is that there is an attenuation factor of 0.4 for every 2 in. of polyethylene or a factor of 0.10 for every 5 in.

Water and wax have also been used as shielding material, with shielding properties similar to polyethylene. Water is slightly less effective on neutrons, but is rarely acceptable because of the adverse safety consequences of a loss of water. Concrete is a better gamma-ray attenuator than the hydrogenous materials already mentioned, but it is much less effective on neutrons.

Borated polyethylene or paraffin can be used to reduce the captured gamma-ray problem and the shield's weight. Neutrons captured in boron produce gamma rays of much lower energy, and a lead skin may not be needed. The extra expense of borated polyethylene can be inhibiting, but other borated compounds are more cost effective.

Slabs of normal polyethylene can be used with interleaved sheets of borated plastic. This is less expensive than borated polyethylene and is quite effective in reducing the capture gamma-ray dose rate while avoiding the lead skin.

Boric acid and beads of high-density polyethylene can be mixed with waterextended polyester to produce a shielding material that is poured into a shell (developed by Frontier Technology, Xenia, Ohio). The resulting material is as good a shield as borated polyethylene, and no lead skin is needed.

Typical thicknesses of these variations of polyethylene are about $2 \mathrm{ft}$ in all directions from the ${ }^{252} \mathrm{Cf}$. An interior attenuator of primary gamma rays detracts from the neutron shielding. If the overall size of the shield is to be small, a primary gamma-ray shield might do more harm than good because the dose rate from neutrons could increase more than the gamma-ray dose rate decreases.

The ${ }^{252} \mathrm{Cf}$ source can be the largest contributor to the neutron background count rate. The amount of shielding between the source and the assay region should take into consideration the size of the background count rate that can be tolerated for the measurement precision requested. Increasing the amount of ${ }^{252} \mathrm{Cf}$ will increase the delayed-neutron count rate, but will also increase the background rate and the shielding problem. Details on the effect of background on the measurement precision are discussed in Sec. IV.B.

4. Design Realization. With the above "physics" calculations completed, the detailed design of the shuffler can proceed.

The mechanical design provides the details needed to fabricate and build the shuffler hardware. If it is found that the assay region musi be modified from the conceptual design, the calculations discussed above may need to be redone. The major mechanical components are the assay region, a storage region, a door or plug, and the ${ }^{252} \mathrm{Cf}$ source transfer system. Various other site-specific accessories may be needed.

The electrical design begins with an outline of the electrical components and their interconnections. Signals into and out of the shuffler must be defined and taken into account. Some signals are internal to the shuffler, but others interact with the installation site in ways specific to the site. 
The software design considers the human, hardware, and electrical requirements, as well as the assay scheme best suited for the situation.

5. Fabrication. The mechanical fabrication proceeds after the mechanical drawings are approved. Some items may need to be tested before leaving the shop (rotation tables, heavy doors).

Many electrical components can be purchased from commercial vendors, but others may have to be custom built. Bench-top testing can avoid many problems that would otherwise be difficult to diagnose after the shuffler is assembled.

Some software modules can be tested before the hardware is available, whereas other modules need prototype or actual hardware.

6. Testing and Calibration. There are several tests to perform on an assembled shuffler. The software options need to be exercised fully with the complete instrument. The optimum operating parameters (for example, irradiation and count times) can be found that minimize the relative precision of the assays (Sec. V.C.2). The shuffler can be calibrated if realistic materials are available.

7. Installation. After the hardware is installed at a site, the components and the whole instrument should be tested and the performance compared to previous experiences. The instrument should be calibrated using actual materials. Operating procedures should be established to get the best results from the instrument and protect it from improper use. As operating experience increases, further studies can be done to enhance this and future shufflers.

\section{IL. THE IDEALIZED CASE}

The following assumptions are made to develop fundamental equations; these constitute an idealized case that is usefully close to reality.

(a) Only one fissionable isotope is in the material being assayed.

(b) All the corresponding time intervals within shuffles are equal (for example, all count times, $t_{c}$, are equal).

(c) The material being assayed is stationary within the assay chamber.

Extensions from these assumptions will be made in the following chapters.

The average fission neutron production rate in the assay material is

$f=v m N_{A} \sigma_{f} \phi / A$, 
where $v$ is the average number of neutrons (including delayed neutrons) released per fission, $m$ is the mass of fissile material, $N_{A}$ is Avogadro's number, $\sigma_{f}$ is the spectrum-weighted fission cross section, $\phi$ is the average irradiating neutron flux and $A$ is the atomic weight of the fissile material. Delayed neutrons from the $j$ th precursor group form a fraction, $\beta_{j}$, of all the fission neutrons.

i igure 8 shows three shuffles (cycles of irradiation and counting) and especially follows the neutrons produced after the irradiation in the first shuffle $(s=1)$. A time $t_{f}$ is needed to move the californium source "forward" into the assay region where it

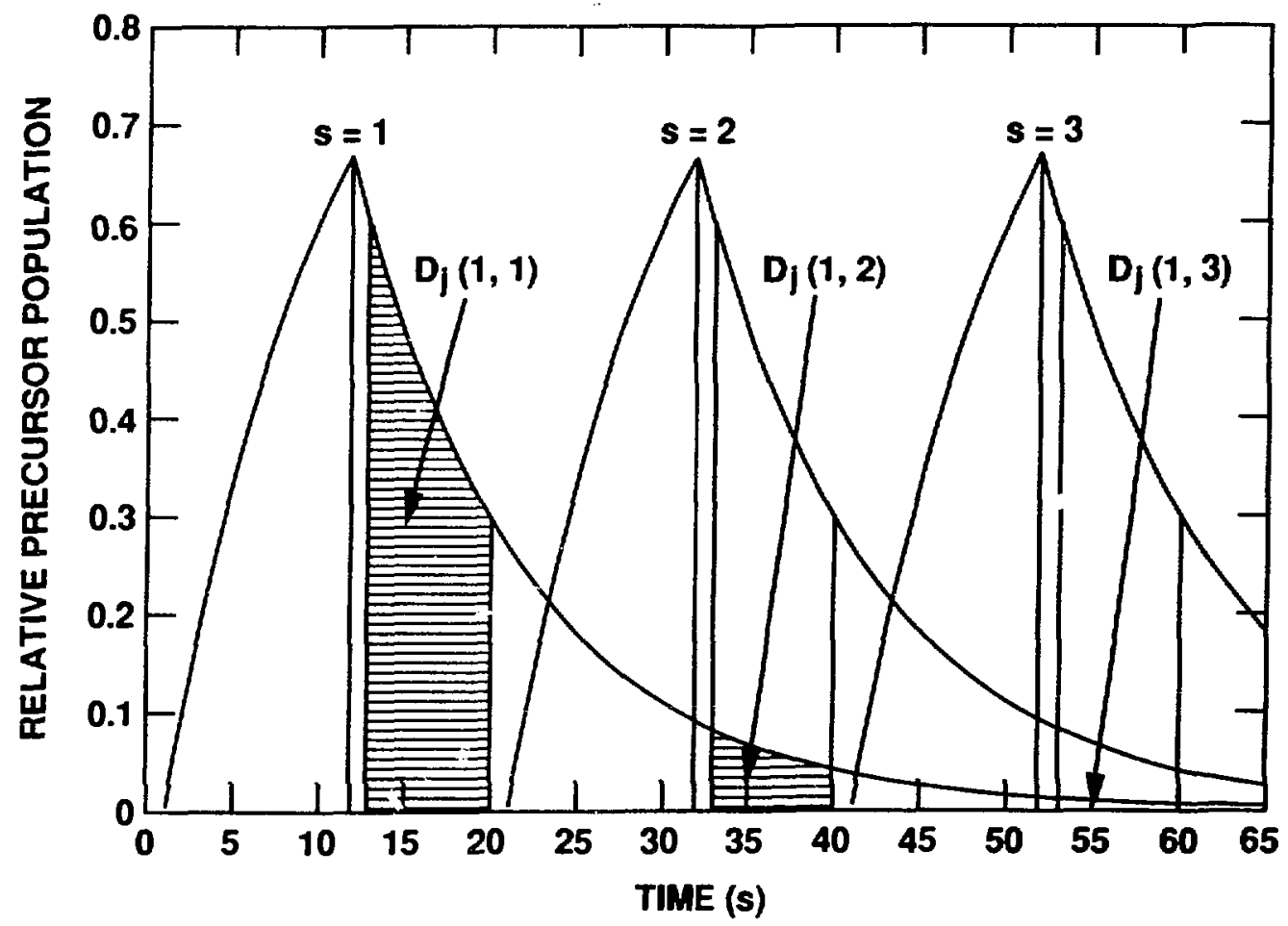

Fig. 8. The contribution of delayed neutrons from the first shuffle $(s=1)$ is traced through the first three shuffles. This figure is for a single precursor (j) with a decay constant of $0.1 \mathrm{~s}^{-1}$.

The forward transfer time $\left(t_{f}\right)$ is shown as $1 \mathrm{~s}$, followed by an 11-s irradiation time $\left(t_{i}\right)$ during which the precursor population grows. At the 12-s time the source is pulled in the reverse direction to the storage location; this time $\left(t_{r}\right)$ is taken to be $1 \mathrm{~s}$. Delayed neutrons are then counted for $t_{c}=7 \mathrm{~s}$, ending at the 20-s mark; the count $D_{j}(1,1)$ is proportional to the shaded area.

The next shuffle $(s=2)$ begins immediately with another forward transfer. During the second count interval ( 33 to $40 \mathrm{~s}$ ) there are still some precursors present from the first shuffle's irradiation. The number of delayed neutrons counted during the second shuffle that were generated by the first shuffle is $D_{j}(1,2)$.

The third shuffle ( $s=3$ ) begins at $40 \mathrm{~s}$ and a very small count $D_{j}(1,3)$ from the first shuffle is found. 
remains for a time $t_{i}$ to irradiate the material. The source is removed in time $t_{r}$ in the "reverse" direction to a storage location. Delayed neutrons are then counted for a time $t_{c}$. The sum of these four times is the period $\tau$ of a shuffle.

\section{A. Delayed Neutrons from Shuffle No. 1}

During the irradiation in the first shuffle, there is both production and decay of precursors. The rate of change of the population of the $j$ th precursor group is

$$
\frac{d P_{j}}{d t}=f \beta_{j}-\lambda_{j} P_{j}
$$

The population of the $j$ th precursor group is thus

$$
P_{j}(t)=\left(\frac{f \beta_{j}}{\lambda_{j}}\right)\left[1-\exp \left(-\lambda_{j} t\right)\right]
$$

At the end of the first irradiation period, the population is

$$
P_{j}\left(t_{i}\right)=\left(\frac{f \beta_{j}}{\lambda_{j}}\right)\left[1-\exp \left(-\lambda_{j} t_{i}\right)\right]
$$

Decay occurs during the delay $t_{r}$ while the irradiation source is being removed, so at the start of the counting interval the population of the $j$ th group is

$$
P_{j}\left(t_{i}+t_{r}\right)=\left(\frac{f \beta_{j}}{\lambda_{j}}\right)\left[1-\exp \left(-\lambda_{j} t_{r}\right)\right]\left[\exp \left(-\lambda_{j} t_{r}\right)\right] .
$$

At any time during the counting interval, the population is

$$
P_{j}(t)=\left(\frac{f \beta_{j}}{\lambda_{j}}\right)\left[\left(1-\exp -\lambda_{j} t_{i}\right)\right]\left[\exp \left(-\lambda_{j} t\right)\right]\left[\exp \left(-\lambda_{j} t\right)\right] .
$$


Figure 9 shows the growth and decay of the six precursor groups from $235 \mathrm{U}$ during and after thermal neutron irradiations of 5 and $10 \mathrm{~s}$ in $235 \mathrm{U}$.

The rates of delayed-neutron production from the six groups are $\lambda_{j} P_{j}$, and these are shown in Fig. 10 for the same single irradiations as in Fig. 9.

The number of delayed neutrons counted during the first shuffle's $t_{c}$ is the time integral of this rate multiplied by the detection efficiency:

$$
\begin{aligned}
D_{j}(1,1) & =\int_{0}^{t} c \lambda_{j} P_{j}(t) d t \\
& =\left(\frac{\varepsilon f \beta_{j}}{\lambda_{j}}\right)\left[1-\exp \left(-\lambda_{j} t_{i}\right)\right]\left[\exp \left(-\lambda_{j} t_{r}\right)\right]\left[1-\exp \left(-\lambda_{j} t_{c}\right)\right],
\end{aligned}
$$

where $D j\left(s_{1}, s_{2}\right)$ denotes the counts during shuffle $s_{2}$ resulting from the irradiation of shuffle $s_{1}$.

During the second shuffle, the precursors from the first shuffle are still producing delayed neutrons (Fig. 5) and the number of these counted in the second counting interval is

$$
D_{j}(1,2)=D_{j}(1,1)\left[\exp \left(-\lambda_{j} \tau\right)\right]
$$

During the sth shuffle, the counts from the first shuffle's irradiation are further diminished to ${ }^{15}$

$$
D_{j}(1, s)=D_{j}(1,1)\left\{\exp \left[-(s-1) \lambda_{j} \tau\right]\right\}
$$

After $n$ shuffles, the total number of delayed-neutron counts from the first shuffle's irradiation is

$$
D_{j}(1, \text { all } n)=D_{j}(1,1) \sum_{s=1}^{n}\left\{\exp \left[-(s-1) \lambda_{j} \tau\right]\right\}
$$




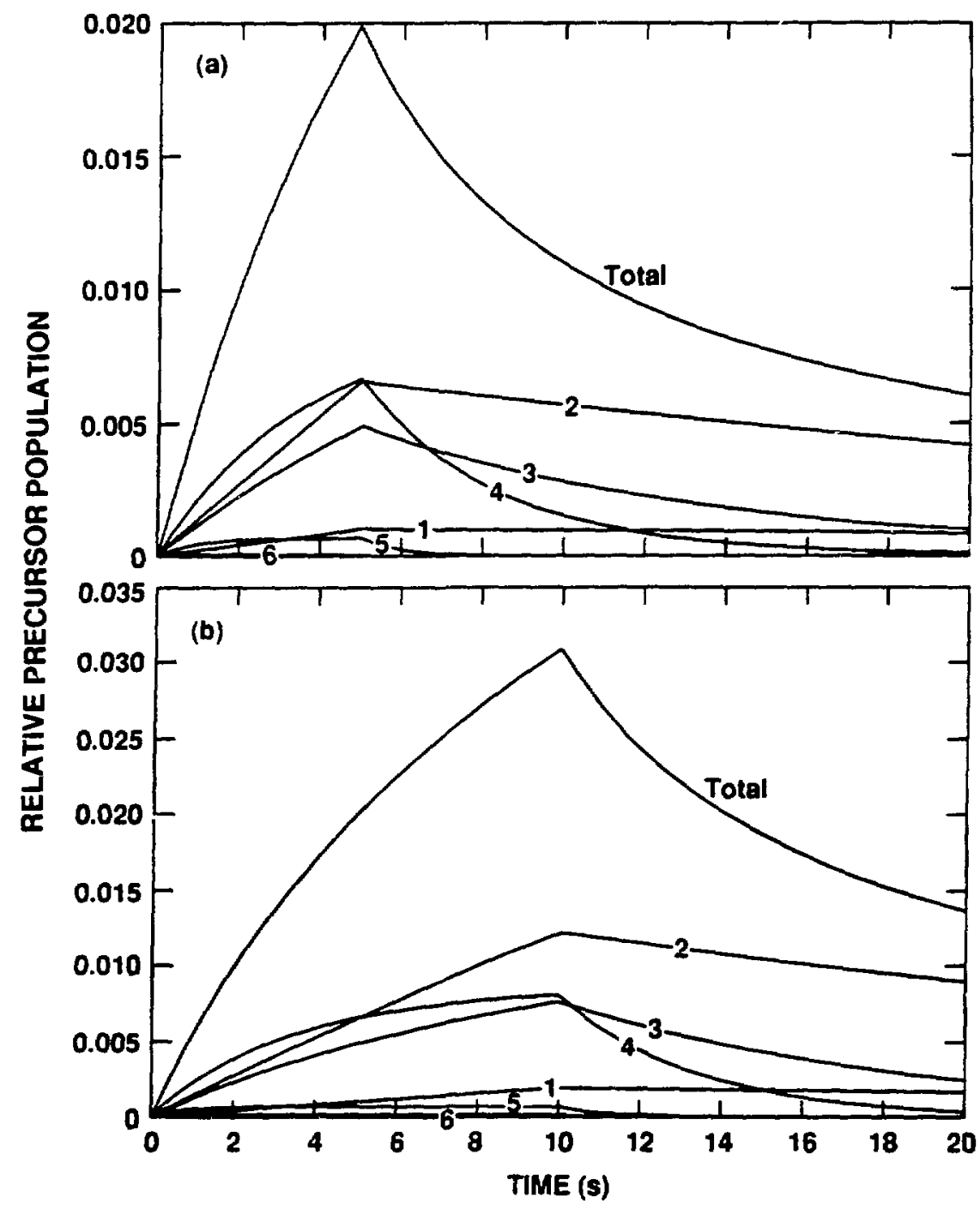

Fig. 9. The data in the Appendix for the delayed-neutron emission following thermal fission of $235 \mathrm{U}$ are used here to illustrate the growth and decay of the six precursors' populations $P_{j}$ and their sum. The samples in Figs. 9(a) and (b) had 5 and 10 s of irradiation, respectively. Precursors 2-4 clearly dominate because group 1 has a very long half-life and grows very slowly, whereas groups 5 and 6 have very short half-lives and reach low-level equilibrium population sizes very quickly. 


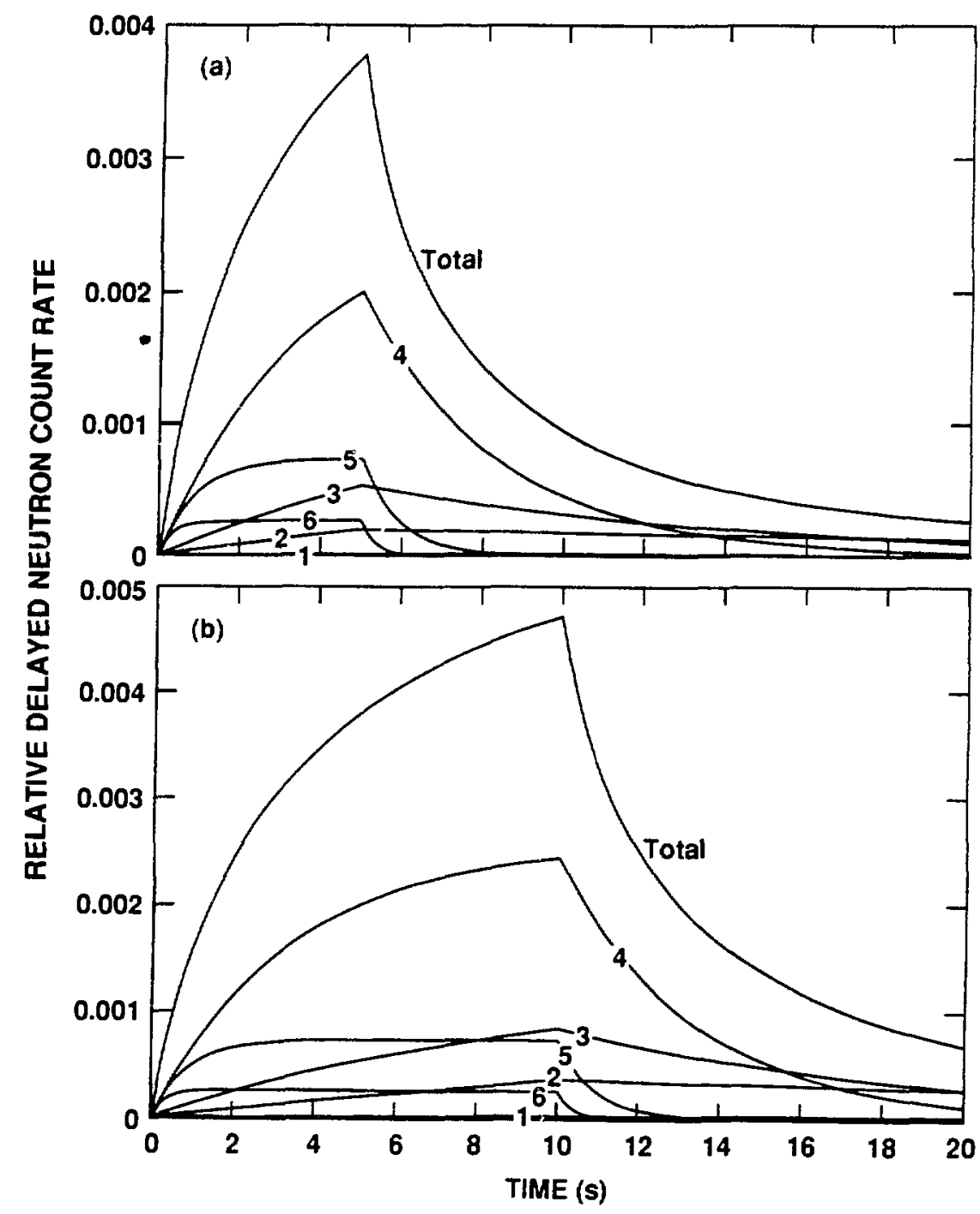

Fig. 10. These two figures are similar to those of Fig. 9 but show the delayed-neutron emission rates instead of the precursor population sizes. Groups 3 and 4 are still very important but groups 2 and 5 have revcised roles. An emission rate is $\lambda_{j} P_{j}$ so, for example, group 5 has both a small population size and a large emission rate because if its relatively large decay constant. 
The sum can be performed, using $q$ for $\exp \left[-\lambda_{j} \tau\right]$ :

$$
\begin{aligned}
\sum_{s=1}^{n} q^{s-1} & =\frac{1}{1-q}-\left(q^{n}+q^{n+1}+\cdots\right) \\
& =\frac{1}{(1-q)}-q^{n}\left(1+q+q^{2}+\ldots\right) \\
& =\frac{1}{(1-q)}-\frac{q^{n}}{(1-q)} \\
& =\frac{\left(1-q^{n}\right)}{(1-q)} .
\end{aligned}
$$

The total of the counts over $n$ shuffles from the first shuffle's irradiation is thus

$$
D_{j}(1, \text { all } n)=D_{j}(1,1) \frac{\left[1-\exp \left(-n \lambda_{j} \tau\right)\right]}{\left[1-\exp \left(-\lambda_{j} \tau\right)\right]}
$$

An example of this function is shown in Fig. 11 for a mixture of uranium isotopes (discussed in Sec. V).

\section{B. Delayed Neutrons in the $n$th Shuffle}

The previous result is also the number of counts during the $n$th shuffle's counting interval from that irradiation and all the previous $(n-1)$ irradiations. Comparing Fig. 12 with Fig. 8 shows why this is so.

$$
D_{j}=D_{j}(1,1) \frac{\left[1-\exp \left(-n \lambda_{j} \tau\right)\right]}{\left[1-\exp \left(-\lambda_{j} \tau\right)\right]}
$$

It has been assumed that all shuffles are identical, so $D_{j}\left(s_{1}+1, s_{2}+1\right)=D_{j}\left(s_{1}, s_{2}\right)$. 


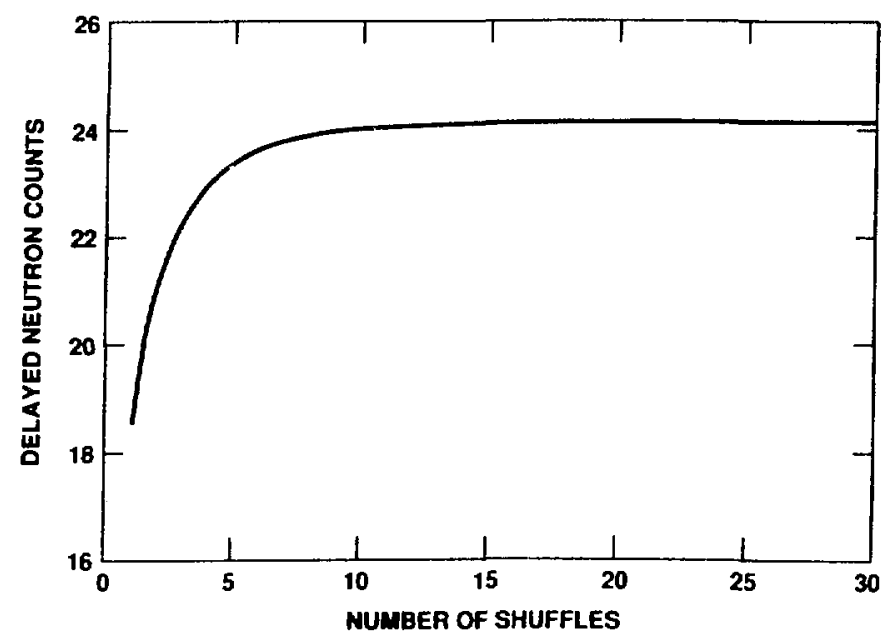

Fig. 11. The number of delayed-neutron counts from the $j$ th precursor after the first irradiation is $D_{j}(1,1)$. As shown in Fig. 8, this irradiation is responsible for counts during the following count intervals, producing the continually decreasing numbers $D_{j}(1,2), D_{j}(1,3), D_{j}(1,4), \ldots$. After $n$ shuffles, there has been a total number of counts $D_{j}(1$, all $n)$ given by $\mathrm{Eq}$. (11), which is plotted here as a function of $n$.

The counts shown here are for forward and reverse transfer times of $1 \mathrm{~s}$ each, with $10 \mathrm{~s}$ of irradiation and $5 \mathrm{~s}$ of counting. A detection efficiency of $25 \%$ is assumed.

Fig. 12. This figure supports the argument that the number of counts in the $n$th counting interval is the same as the sum of the counts from the first irradiation during the $n$ counting intervals. During the third shuffle ( $s=3$ ), for example, the three contributors to the count, $D_{j}(3,3)$, $D_{j}(2,3), D_{j}(1,3)$, are numerically the same as $D_{j}(1,1), D_{j}(1,2), D_{j}(1,3)$ from Fig. 8 , respectively.

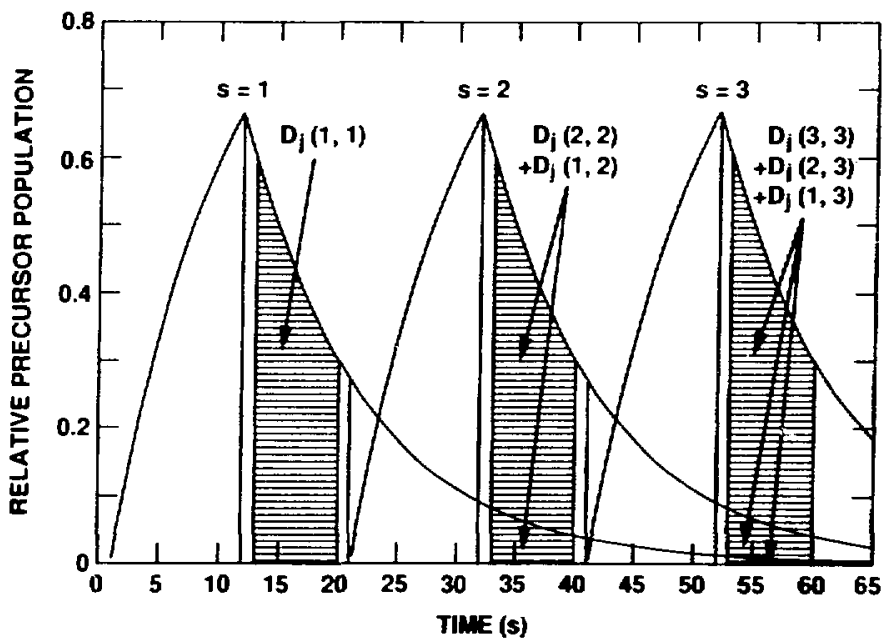

The number of counts in any one shuffle approaches an equilibrium value as the number of shuffles increases. The equilibrium value is

$$
D_{j}=\frac{D_{j}(1,1)}{\left[1-\exp \left(-\lambda_{j} \tau\right)\right]} .
$$

Figure 11 is thus also an example of Eq. (12) with all precursors taken into account; the equilibrium value of Eq. (13) is nearly reached after a dozen shuffles. 
The production rate of delayed neutrons during multiple shuffles is illustrated in Fig. 13, where the total rate is shown along with the individual precursors.

\section{Delayed Neutrons from $n$ Shuffles}

In an assay, $n$ shuffles are made and delayed neutrons are counted during each count time $t_{c}$ of each shuffle. The sum of all these counts for a single precursor group is based on Eq. (12):3,16

$$
D_{j}=D_{j}(1,1) \sum_{s=1}^{n} \frac{1-\exp \left(-s \lambda_{j} \tau\right)}{1-\exp \left(-\lambda_{j} \tau\right)}
$$

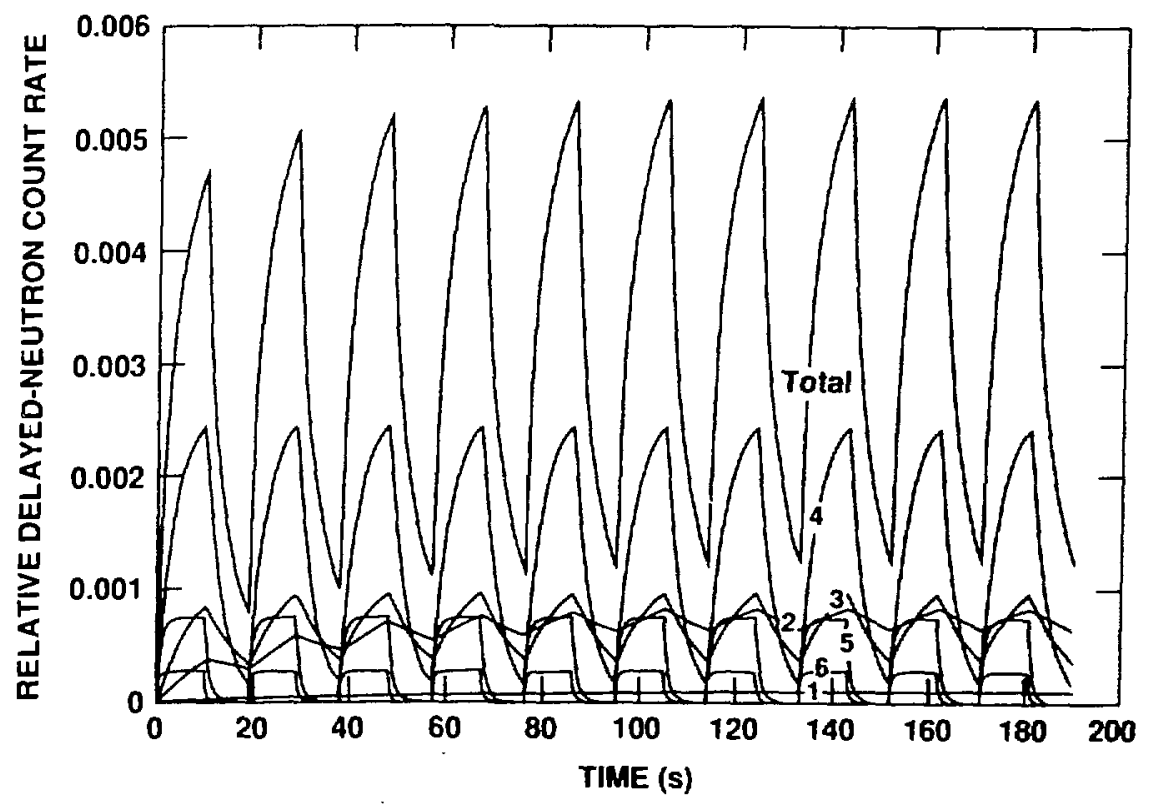

Fig. 13. The growth and decay of each delayed-neutron emission rate is shown for all six precursor groups and their sum for 10 shuffles. The forward and reverse transfer times are $1 \mathrm{~s}$, the irradiation time is $10 \mathrm{~s}$, and the count time is $7 \mathrm{~s}$.

The emission rates from groups 4, 5, and 6 with short half-lives quickly reach equilibrium values. Group 1, with a long half-life, continually grows but gives an inconsequential emission rate. Group 3 is essentially at equilibrium after one shuffle, but group 2 takes several shuffles to get close to equilibrium. The total emission rate thus grows significantly for a few cycles and then stabilizes. 
This sum can be performed, using $q \equiv \exp \left(-\lambda_{j} \tau\right)$ temporarily:

$$
\begin{aligned}
\sum_{s=1}^{n} \frac{1-q^{s}}{1-q} & =\frac{1}{1-q}\left(n-q \sum_{s=1}^{n} q^{s-1}\right) & =\frac{1}{1-q}\left(n-q \frac{1-q^{n}}{1-q}\right) \\
& =\frac{n}{1-q}-\frac{q\left(1-q^{n}\right)}{(1-q)^{2}} & =\frac{n-(n+1) q+q^{n+1}}{(1-q)^{2}} .
\end{aligned}
$$

The counts from precursor $j$ from all $n$ shuffles is thus

$$
D_{j}=D_{j}(1,1) \frac{\left[n-(n+1) q+q^{n+1}\right]}{(1-q)^{2}}
$$

The counts from all precursors during $n$ shuffles is ${ }^{17}$

$$
\begin{aligned}
D & =\sum_{j=1}^{6} D_{j} \\
& =\sum_{j=1}^{6}\left(\frac{\varepsilon f \beta_{j}}{\lambda_{j}}\right)\left[1-\exp \left(-\lambda_{j} t_{i}\right)\right]\left[\exp \left(-\lambda_{j} t_{r}\right)\right]\left[1-\exp \left(-\lambda_{j} t_{c}\right)\right] \\
& \cdot\left\{\frac{n}{1-\exp \left(-\lambda_{j} \tau\right)}-\frac{\exp \left(-\lambda_{j} \tau\right)\left[1-\exp \left(-n \lambda_{j} \tau\right)\right]}{\left[1-\exp \left(-\lambda_{j} \tau\right)\right]^{2}}\right\} \\
= & \sum_{j=1}^{6}\left(\frac{\varepsilon f \beta_{j}}{\lambda_{j}}\right)\left[1-\exp \left(-\lambda_{j} t_{i}\right)\right]\left[\exp \left(-\lambda_{j} t\right)\right]\left[1-\exp \left(-\lambda_{j} t_{c}\right)\right] \\
& \cdot\left\{\frac{n-(n+1) \exp \left(-\lambda_{j} t\right)+\exp \left[-(n+1) \lambda_{j} \tau\right]}{\left[1-\exp \left(-\lambda_{j} \tau\right)\right]^{2}}\right\} .
\end{aligned}
$$


Notice that if there is only one shuffle $(n=1)$, the last factor in Eq. (17) is one and Eq. (17) is the same as the sum of Eq. (6) over the precursor groups. The number of counts $D$ as a function of the number of shuffles $n$ is shown in Fig. 14 for a particular set of circumstances.

When evaluating Eq. (17) the values of $\beta_{j}$ for some isotope may not be readily available. Values of $\beta_{j} v$ might be provided, which are sufficient because fincludes $v$ as a factor.

With a background rate of $b$ counts per second, the total measured count rate is $c=D / T_{c}+b$, where $T_{c}$ is the total count time $n t_{c}$. More discussion of background effects is given in Sec. V.

Another way to express $D_{j}$ of Eq. (16) is given in Ref. 18. The terms in the sum of Eq. (14) are added in a different order. Figure 15 shows the $j$ th precursor's counts from the first three and the last shuffle in an assay. Each shuffle contributes $D_{j}(1,1)$ counts, for a total of $n D_{j}(1,1)$ counts. Each shuffle except the last contributes counts in the succecding shuffle's count interval; this amounts to a total of $(n-1) D_{j}(1,1) \exp \left(-\lambda_{j} t\right)$ counts.

This process continues until there is only the last counting interval for the first shuffle, which yields $D_{j}(1,1) \exp \left[-(n-1) \lambda_{j} t\right]$ counts.

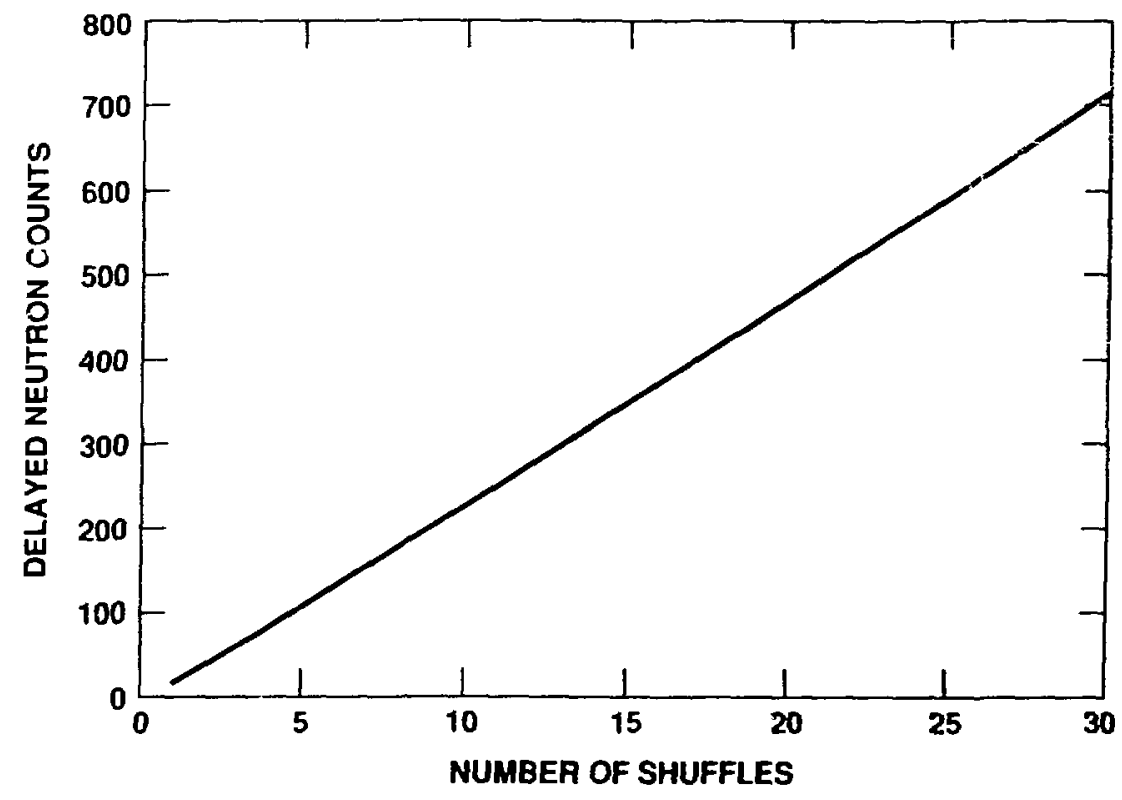

Fig. 14. The number of counts after $n$ shuffles is nearly linear with $n$. This example has a transfer time of $1 \mathrm{~s}$, an irradiation time of $10 \mathrm{~s}$, and a count time of $5 \mathrm{~s}$. There is a slight nonlinearity during the first few shuffles because the precursor populations have not yet built to equilibrium sizes, as indicated in Fig. 13. 


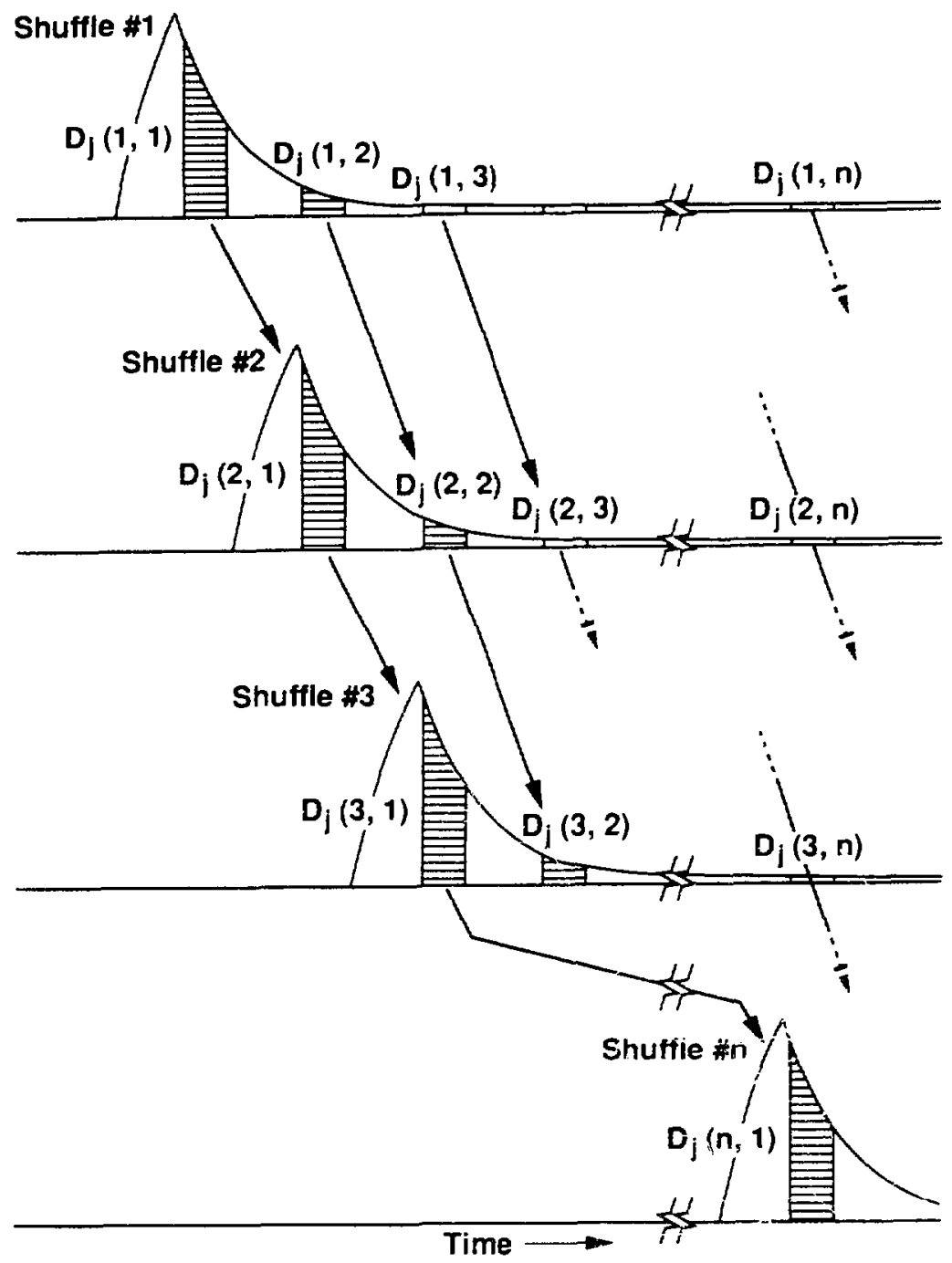

Fig. 15. The terms in the sum $D_{j}(n)$ are displayed here to show their sum in a visual manner. The first three and the last shuffles are shown explicitly. The slanted arrows show the sequence of the terms.

Each shuffle contributes a count equal to $D_{j}(1,1)$ immediatcly after an irradiation because $D_{j}(n, 1)=D_{j}(3,1)=D_{j}(2,1)=D_{j}(1,1)$. The sum of these is $n D_{j}(1,1)$.

Each shuffle except the $n$th also contributes counts during the following shuffle. Each count is $D_{j}(1,1) \exp \left(-\lambda_{j} \tau\right)$ and there are $(n-1)$ of them, so their sum is $(n-1) D_{j}(1,1) \exp \left(-\lambda_{j} \tau\right)$.

Each shuffle except the $n$th and the $(n-1)$ st contributes during the second shuffle following its own irradiation. This forms a sum of $(n-2) D_{j}(1,1) \exp \left(-2 \lambda_{j} \tau\right)$.

Generalizing this process to the sth term, there are $(n-s+1)$ contributors, each giving an amount $D_{j}(1,1) \exp \left[-(s-1) \lambda_{j} \tau\right]$. The sum of $(n-s+1) D_{j}(1,1) \exp \left[-(s-1) \lambda_{j} \tau\right]$ over $s$ from 1 through $n$ is thus the total number of delayed-neutron counts. 
The sum of all these counts, arranged in this manner, is

$$
D_{j}=D_{j}(1,1) \sum_{s=1}^{n}(n-s+1) \exp \left[-(s-1) \lambda_{j} \tau\right]
$$

Equations (14), (16), and (18) are equivalent. The various counts shown in Fig. 15 are simply being added in different orders to get Eqs. (16) and (18). Equation (16) uses counts that would actually be measured during the different count intervals and then adds them all together; Eq. (18) first adds identical counts that are found at different count intervals. Using directions on Fig. 15, Eq. (16) first adds counts on the same "vertical" lines (same times), then adds all the counts found from the different vertical lines; Eq. (18) adds identical counts that form "diagonal" lines (as shown by the arrows), then adds the counts found from the different diagonal lines.

An algebraic way to show the equivalence of Eqs. (14) and (18) starts with the recognition from Eq. (10) that $\left(1-q^{s}\right) /(1-q)$ is the sum of this geometric series: $1+q+$ $q^{2}+q^{3}+\ldots+q^{s-1}$. The sum in Eq. (15) can thus be expanded in a sum of geometric series and then recombined into Eq. (18):

$$
\begin{array}{rlr}
\sum_{s-1}^{n} \frac{1-q^{s}}{1-q}= & 1 \quad(s=1) \\
& +1+q \quad(s=2) \\
& +1+q+q^{2} \quad(s=3) \\
& +1+q+q^{2}+q^{3} \quad \quad(s=4) \\
& +\cdots & \cdots \\
= & n+(n-1) q+(n-2) q^{2}+(n-3) q^{3}+\ldots+q^{n-1} \\
= & \sum_{s=1}^{n}(n-s+1) q^{-(s-1)} .
\end{array}
$$

\section{Countable Fraction}

Only a fraction of all the delayed neutrons produced are counted because the counters are off during the irradiation and while the source is being moved, and the chosen count time does not allow the last small fraction of delayed neutrons to he counted.

Equation (17) gives the number of delayed neutrons that are counted during $n$ shuffles. The maximum number of delayed neutrons that could have been counted during these $n$ shuffles (had counting been continuous) is $\varepsilon f t_{i} \cdot \Sigma \beta_{i}$. The factor $f t_{i}$ is the number of fission neutrons produced and $\Sigma \beta_{j}$ is the fraction of the fission 
neutrons that are delayed, so $f t_{i} \cdot \Sigma \beta_{j}$ is the number of delayed neutrons produced. These are then counted with an efficiency of $\varepsilon$.

The countable fraction is thus Eq. (17) divided by $\varepsilon f t_{i} \cdot \Sigma \beta_{j}$. The fraction is independent of the detection efficiency $\varepsilon$ and the fission rate $f$. Examples of how the countable fraction varies with some of the parameters are given in Fig. 16.

\section{E. Maximizing Delayed-Neutron Counts}

When a fixed amount of time is available for an assay, the irradiation and counting times, $t_{i}$ and $t_{c}$, can be selected to maximize the number of counts during an assay. ${ }^{18}$ It will now be shown that this occurs when $t_{i}$ and $t_{c}$ are equal.

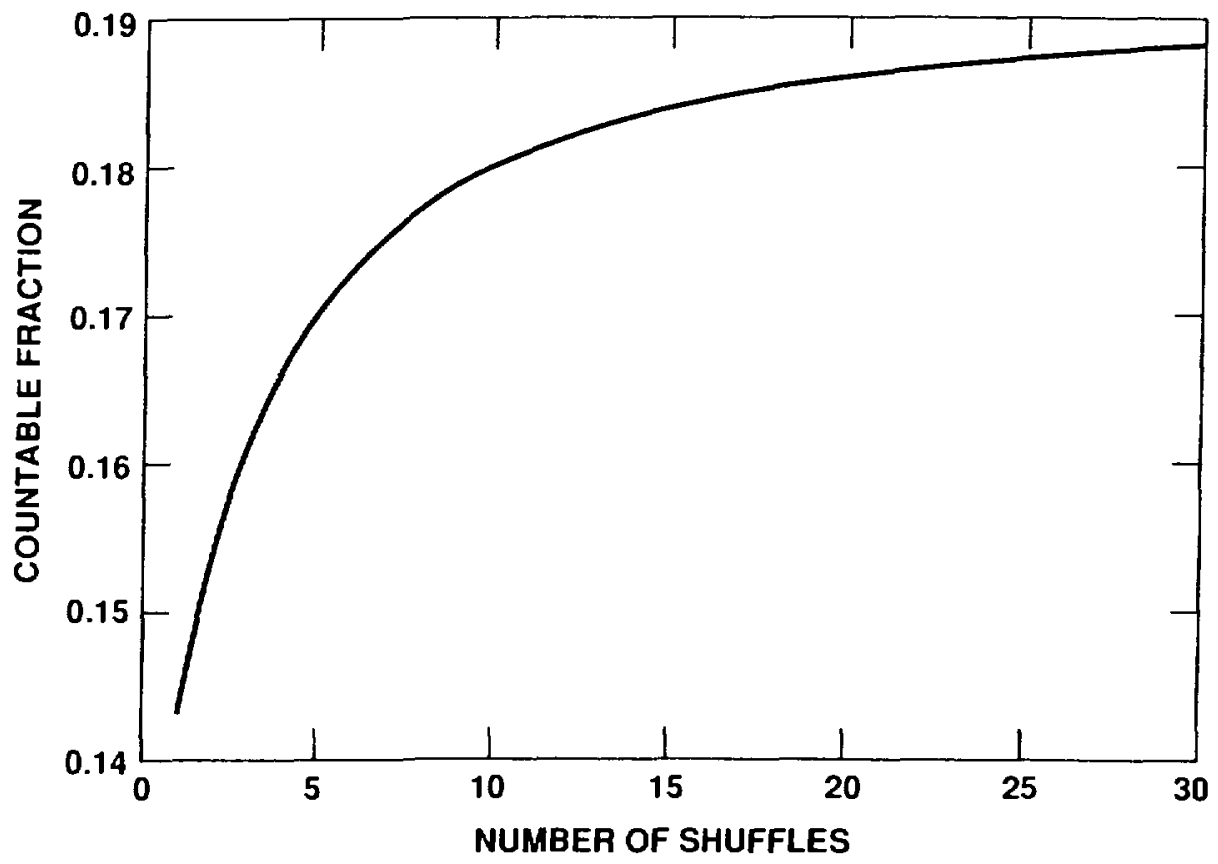

Fig. 16. Not all the delayed neutrons produced during $n$ shuffles can be counted, even with a $100 \%$ efficient detector: delayed neutrons cannot be counted during the irradiations; the forward and reverse transfer times are not zero and neutrons are not counted during these times; a new irradiation is begun well before all the precursors from the previous shuffles have decayed. As may be inferred from Fig. 12, the average count rate can be increased by using a series of many short count periods instead of a few long ones.

This graph shows the fraction of delayed neutrons produced that are counted as a function of the number of shuffles. This assumes irradiation times of $10 \mathrm{~s}$, count times of $5 \mathrm{~s}$, and transfer times of $1 \mathrm{~s}$ each. A mixture of uranium isotopes was used: $50 \%{ }^{235} \mathrm{U}$ (thermal fission), $33 \%{ }^{235} \mathrm{U}$ (fast fission), and $17 \%{ }^{238} \mathrm{U}$ (fast fission). The detection efficiency was taken to be $25 \%$.

The increase in the fraction with the number of shuffles is related to the increasing number of delayed neutrons counted as the number of shuffles grows (Figs. 13 and 15). 
Equation (17) gives the number of counts for any combination of times. The source transfer times, $t_{f}$ and $t_{r}$ are made as short and reproducible as possible by the shuffler mechanism and are thus constants. The time for a shuffle $\tau$ will also be taker as a constant and the optimum $t_{i}$ and $t_{c}$ will be expressed in terms of

$$
\tau^{\prime} \equiv \tau-\left(t_{f}+t_{r}\right)=t_{i}+t_{c}
$$

Equation (17) can be made into a function of only $t_{c}$ by setting $t_{i}=\tau^{\prime}-t_{c}$. The maximum value of $D$ is found from $\partial D / \partial t_{C}=0$. Each term in the sum of derivatives over $j$ is independent (because the $\lambda_{j}$ are independent) and thus must be zero individually: $\partial D_{j} / \partial t_{c}=0$. This derivative reduces to

$$
\begin{aligned}
& \exp \left(-\lambda_{j} t_{c}\right)\left[1-\exp \left(-\lambda_{j} \tau^{\prime}\right) \cdot \exp \left(\lambda_{j} t_{c}\right)\right] \\
& -\exp \left(-\lambda_{j} \tau^{\prime}\right) \cdot \exp \left(\lambda_{j} t_{c}\right) \cdot\left[1-\exp \left(-\lambda_{j} t_{c}\right)\right]=0 .
\end{aligned}
$$

This equation for $\exp \left(-\lambda_{j} t_{c}\right)$ gives $t_{c}=\tau^{\prime} / 2=\left(\tau-t_{f}+t_{r}\right) / 2$, which requires that $t_{i}=t_{c}$ regardless of the values of $\tau, t_{f}$, and $t_{r}$. This conclusion holds for each precursor $j$.

Another way to reach the same conclusion is to note that the essential part of $D_{j}$ in Eq. (17) is $\left[1-\exp \left(-\lambda_{j} t_{c}\right)\right] \cdot\left(1-\exp \left[-\lambda_{j}\left(\tau^{\prime}-t_{c}\right)\right]\right\}$. This is a symmetric function of $t_{c}$ about $t_{c}=\tau / 2$, where it has a maximum. By using $t_{i}=t_{c}$ the number of delayed-neutron counts is maximized.

If it is preferred to minimize the count precision, the choices of $t_{i}$ and $t_{c}$ depend on the background count rate. This is discussed in Sec. V.C.2.

\section{IRREGULAR SHUFFLE TIMES AND NUMBERS}

The equations for the idealized case assume that the shuffles' timing never varies. This can be only approximated in practice. Furthermore, calibration may have been completed with one set of shuffles and timings, but later another set might be preferred (for example, to perform assays more quickly). The calibration curve no longer applies unless the measurements are corrected.

The relative number of neutrons counted in a sequence of shuffles forming an assay can be calculated for the ideal times and also from the actual, slightly irregular, times for each shuffle. A ratio of relative numbers for the ideal and actual times can be used to correct for the irregular times. The measured data are then adjusted to values expected from regular times. The behavior of the instrument is also monitored through the size of the adjustment. 
The number of shuffles in an assay will normally be the same from assay to assay, but if this is not so, an adjustment for different numbers of shuffles is also made by this ratio of calculated and measured quantities.

All assays can use the same calibration curve as long as the count rates from irregular assays are converted into equivalent count rates for a standard assay scheme.

\section{A. Delayed-Neutron Counts with Irregular Times}

If all the times during an assay are the same from shuffle to shuffle, the number of delayed-neutron counts is given by Eq. (17). In practice, the times can show variations, although their differences (a few milliseconds) are not significant. Each individual time during an assay can be measured and used to adjust the measured count rate to what would be expected had all the times been regular. Although the small adjustment will not normally affect the assay result, knowing that it is small gives confidence that the instrument functioned properly during the assay. The adjustment size can be used to call attention to an anomalous assay procedure, should that be necessary.

An iterative expression for the number of counts useful in computer codes will be developed in a stepwise fashion. All the individual time segments will be carefully considered because of their irregular natures.

The number of counts from the $j$ th group during the first shuffle was already given in Eq. (6):

$$
\begin{aligned}
D_{j}(1) & =D_{j}(1,1) \\
& =\left(\frac{\varepsilon f \beta_{j}}{\lambda_{j}}\right)\left[1-\exp \left(-\lambda_{j} t_{i 1}\right)\right]\left[\exp \left(-\lambda_{j} t_{r 1}\right)\right]\left[1-\exp \left(-\lambda_{j} t_{c 1}\right)\right] .
\end{aligned}
$$

The subscripts on the times indicate that they are for the first shuffle.

During the second shuffle, with a second set of times, there are contributions from the first and second irradiations:

$$
\begin{aligned}
D_{j}(2)= & D_{j}(1,2)+D_{j}(2,2) \\
= & \left(\frac{\varepsilon f \beta_{j}}{\lambda_{j}}\right)\left[1-\exp \left(-\lambda_{j} t_{i 1}\right)\right]\left[\exp \left(-\lambda_{j} t_{r 1}\right)\right] \\
& \cdot\left(\exp \left[-\lambda_{j}\left(t_{c 1}+t_{f 2}+t_{i 2}+t_{r 2}\right)\right]\right\}\left[1-\exp \left(-\lambda_{j} t_{c 2}\right)\right] \\
& +\left(\frac{\varepsilon f \beta_{j}}{\lambda_{j}}\right)\left[1-\exp \left(-\lambda_{j} t_{i 2}\right)\right]\left[\exp \left(-\lambda_{j} t_{r 2}\right)\right]\left[1-\exp \left(-\lambda_{j} t_{c 2}\right)\right] .
\end{aligned}
$$


During shuffle no. 3 , there are three contributors:

$$
\begin{aligned}
D_{j}(3)= & D_{j}(1,3)+D_{j}(2,3)+D_{j}(3,3) \\
= & \left(\frac{\varepsilon f \beta_{j}}{\lambda_{j}}\right)\left[1-\exp \left(-\lambda_{j} t_{i 1}\right)\right]\left[\exp \left(-\lambda_{j} t_{r 1}\right)\right] \\
& \cdot\left\{\exp \left[-\lambda_{j}\left(t_{c 1}+t_{f 2}+t_{i 2}+t_{r 2}\right)\right]\right\} \\
& \cdot\left\{\exp \left[-\lambda_{j}\left(t_{c 2}+t_{f 3}+t_{i 3}+t_{r 3}\right)\right]\right\} \cdot\left[1-\exp \left(-\lambda_{j} t_{c 3}\right)\right] \\
& +\left(\frac{\varepsilon f \beta_{j}}{\lambda_{j}}\right)\left[1-\exp \left(-\lambda_{j} t_{i 2}\right)\right]\left[\exp \left(-\lambda_{j} t_{r 2}\right)\right] \\
& \cdot\left\{\exp \left[-\lambda_{j}\left(t_{c 2}+t_{f 3}+t_{i 3}+t_{r 3}\right)\right]\right\}\left[1-\exp \left(-\lambda_{j} t_{c 3}\right)\right] \\
& +\left(\frac{\varepsilon f \beta_{j}}{\lambda_{j}}\right)\left[1-\exp \left(-\lambda_{j} t_{i 3}\right)\right]\left[\exp \left(-\lambda_{j} t_{r 3}\right)\right]\left[1-\exp \left(-\lambda_{j} t_{c 3}\right)\right] .
\end{aligned}
$$

Rather than continue this awkward process, a set of iterative expressions can be used, especially in a computer code. ${ }^{19}$ At the start of the count interval of the sth shuffle, the precursor population of group $j, P_{j s}$, has contributions from the present shuffle and all previous shuffles:

$$
P_{j s}=\left(\frac{f \beta_{j}}{\lambda_{j}}\right)\left[1-\exp \left(-\lambda_{j} t_{i s}\right)\right]\left[\exp \left(-\lambda_{j} t_{r s}\right)\right]+P_{j, s-1}^{\prime} \cdot \exp \left[-\lambda_{j}\left(t_{f s}+t_{i s}+t_{r s}\right)\right]
$$

The $P_{j, s^{-1}}^{\prime}$ factor is the precursor population from all previous shuffles at the end of the $s$ - 1 counting interval; its exponential multiplier reduces the population size to the start of the sth counting interval. For the first shuffle, $P_{j, 0}{ }^{0}=0$. 
The number of delayed neutrons recorded during this current counting interval is the integral of $\varepsilon \lambda_{j} P_{j s} \cdot\left[\exp \left(-\lambda_{j} t_{c}\right)\right]$ from $t_{c}=0$ to $t_{c s}$ :

$$
D_{j}(s)=\varepsilon P_{j s} \cdot\left[1-\exp \left(-\lambda_{j} t_{c s}\right)\right]
$$

In preparation for the $s+1$ shuffle, $P_{j s}^{\prime}$ can be calculated with the current shuffle's times:

$$
\begin{aligned}
P_{j s}^{\prime} & =P_{j, s-1}^{\prime} \cdot\left\{\exp \left[-\lambda_{j}\left(t_{f s}+t_{i s}+t_{c s}+t_{r s}\right)\right]\right\} \\
& +\left(\frac{\varepsilon f \beta_{j}}{\lambda_{j}}\right)\left\{\exp \left[-\lambda_{j}\left(t_{r s}+t_{c s}\right)\right]\right\}\left[1-\exp \left(-\lambda_{j} t_{i s}\right)\right] .
\end{aligned}
$$

The total counts from group $j$ in $n$ shuffles is the sum of the $D_{j}(s)$ :

$$
D_{j}=\sum_{s=1}^{n} D_{j}(s)
$$

After each shuffle, the items in Eqs. (24)-(27) are calculated. When the last shuffle is completed, the count of delayed neutrons is

$$
D=\sum_{j=1}^{6} D_{j} .
$$

\section{B. Adjusting Counts For Irregular Times}

The measured count can be adjusted for irregular times using the above equations. The ratio of Eqs. (17) and (28) compares the uniform case to the irregular case and is independent of detection efficiency and fission rate; this is called the cycle correction 
factor $C F_{\text {cycle. }}$ The measured count multiplied by this ratio is the count that would have been obtained had all the times been uniform.

$$
D_{\text {unif }}=D_{\text {irr }} \frac{\text { Eq. }(17)}{\text { Eq. (28) }}=D_{\text {irr }} C F_{\text {cycle }}
$$

\section{Adjusting Counts for Numbers of Shuffles per Assay}

Different assays may use different numbers of shuffles. A correction must be made before these counts can be compared, as with a single calibration curve.

The procedure is to define a standard number of shuffles in an assay and adjust the measured data to that number. Equation (17) is used to calculate the expected counts (aside from detection efficiency and fission rate) for the standard number of shuffles and for uniform times. The cycle correction factor in Eq. (29) then corrects not only for timing irregularities, but also for deviations from the standard number of shuffles because Eq. (28) uses only the actual number of shuffles made.

\section{BACKGROUND, PRECISION, AND DETECTION LIMIT}

\section{A. Background Sources}

Background counts can arise from several sources. The ${ }^{252} \mathrm{Cf}$ source is not likely to be perfectly shielded from the assay region and can produce a measurable count rate even when in the storage position. Cosmic-ray products interact with materials in and around the shuffler to generate neutrons that may be counted. The material being assayed may produce neutrons through spontaneous fissions or $(\alpha, n)$ reactions in addition to the neutrons released during the induced fissions.

\section{B. Counting Precision and Detection Limit}

Whatever the source of background neutrons, they must be taken into account when computing the precision of the delayed-neuiron counting. The delayed-neutron counts in an assay (consisting of $n$ shuffles) is $D$, but the number of neutron counts measured is $C$, which includes a number, $B$, of background neutrons. The background, $B$, for the total delayed-neutron count time, $T_{C}$, is estimated from a separate background count that gave $B_{b}$ counts in a time $t_{b} .16,20,21$

$$
D=C-B \approx C-B_{b}\left(\frac{T_{C}}{t_{b}}\right) \text {. }
$$


The relative precision of $D$ is

$$
\frac{\sigma_{D}}{D}=\frac{\sqrt{\sigma_{C}^{2}+\sigma_{B}^{2}}}{D}
$$

But $\sigma_{C}=\sqrt{C}=\sqrt{D+B_{b}}\left(\overline{T_{C} / t_{b}}\right)$ and $\sigma_{B}=\sqrt{B_{b}} \cdot\left(T_{C} / t_{b}\right)$, so the relative precision is

$$
\frac{\sigma_{D}}{D}=\frac{\sqrt{D+B_{b}\left(\frac{T_{C}}{t_{b}}\right)+B_{b}\left(\frac{T_{C}}{t_{b}}\right)^{2}}}{D}
$$

The background rate from spontaneous fissions in uranium is quite low and $\sigma_{D} / D$ is also low even for small quantities of uranium. A gram of low-enriched uranium with $t_{b}=300 \mathrm{~s}$ might give $B_{b}=1$, whereas $D$ after $T_{C}=3200 \mathrm{~s}$ could be 450 . In this case, $s_{D} / D$ is a rather low $4.7 \%$. Any other sources of background would increase this relative precision.

A gram of plutonium with $6 \% 240 \mathrm{Pu}$ measured under the same conditions could have $B_{b}=16500$ counts, $D=150$ counts, and thus $\sigma_{D}=121 \%$, which is well outside the generally used goal of $33 \%$ or less. Other likely sources of background are probably negligible. For $15 \mathrm{~g}$ of plutonium, the value of $\sigma_{D} / D$ drops to an acceptable $31 \%$.

The high backgrounds generated by plutonium make it more difficult to assay than uranium using a shuffler. The instrument can respond to the very high background count rate by informing the user that plutonium may be present. If the isotopics are then provided, the passive background count may give a more precise assay than the active interrogation.

If a certain relative precision is to be achieved, a minimum number of delayedneutron counts $D$ must be collected. Equation (32) can be solved for the minimum $D$ required:

$$
D=\frac{1+\sqrt{1+4\left(\frac{\sigma_{D}}{D}\right)^{2} B_{b}\left(\frac{T_{C}}{t_{b}}\right)\left(1+\frac{T_{C}}{t_{b}}\right)}}{2\left(\frac{\sigma_{D}}{D}\right)^{2}}
$$

For example, assume the desired precision is $2 \%$ and 200 background counts were obtained during a time of $t_{b}=300 \mathrm{~s}$. If the total count time during the assay phase is 
$T_{C}=245 \mathrm{~s}$, then the required number of delayed-neutron counts is $D=2768$. By relaxing the precision to $10 \%$, the required number of delayed-neutron counts is only 229.

This equation is evaluated in Fig. 17 to show $D$ as a function of $B_{b}$, with $D / \sigma_{D}=3$ and $T_{C} / t_{b}$ as a parameter. There is obvious value in reducing the background as much as possible. For a given background count, the required value of $D$ can be reduced by using a smaller value of $T_{C} / t_{b}$; there would then be a lower background count rate and $t_{b}$ must be increased to get the specified background count. However, this conclusion is simplistic because only a finite length of time is available to count background and delayed neutrons; this time cannot be used entirely for a background count. How to divide the time available between the delayed-neutron and background counts is discussed in Sec. V.C.2.

If the calibration parameters have been measured oi estimated, the counts, $D$, of Eq. (33) can be converted into a mass. This mass is the detection limit of the instrument for the given $\left(D / \sigma_{D}\right)$ and other parameters in Eq. (33). A commonly used criterion is to have the signal at least $3 \sigma$ above background, which implies that $D / \sigma_{D}>3$ or $\sigma_{D} / D<0.333$ (or $33 \%$ ). This criterion was used in the examples of relative precisions from uranium and plutonium earlier in this section.

As smaller precisions are imposed, greater numbers of delayed neutrons must be generated, which probably means a larger ${ }^{252} \mathrm{Cf}$ source. It might seem that $B_{b}$ would

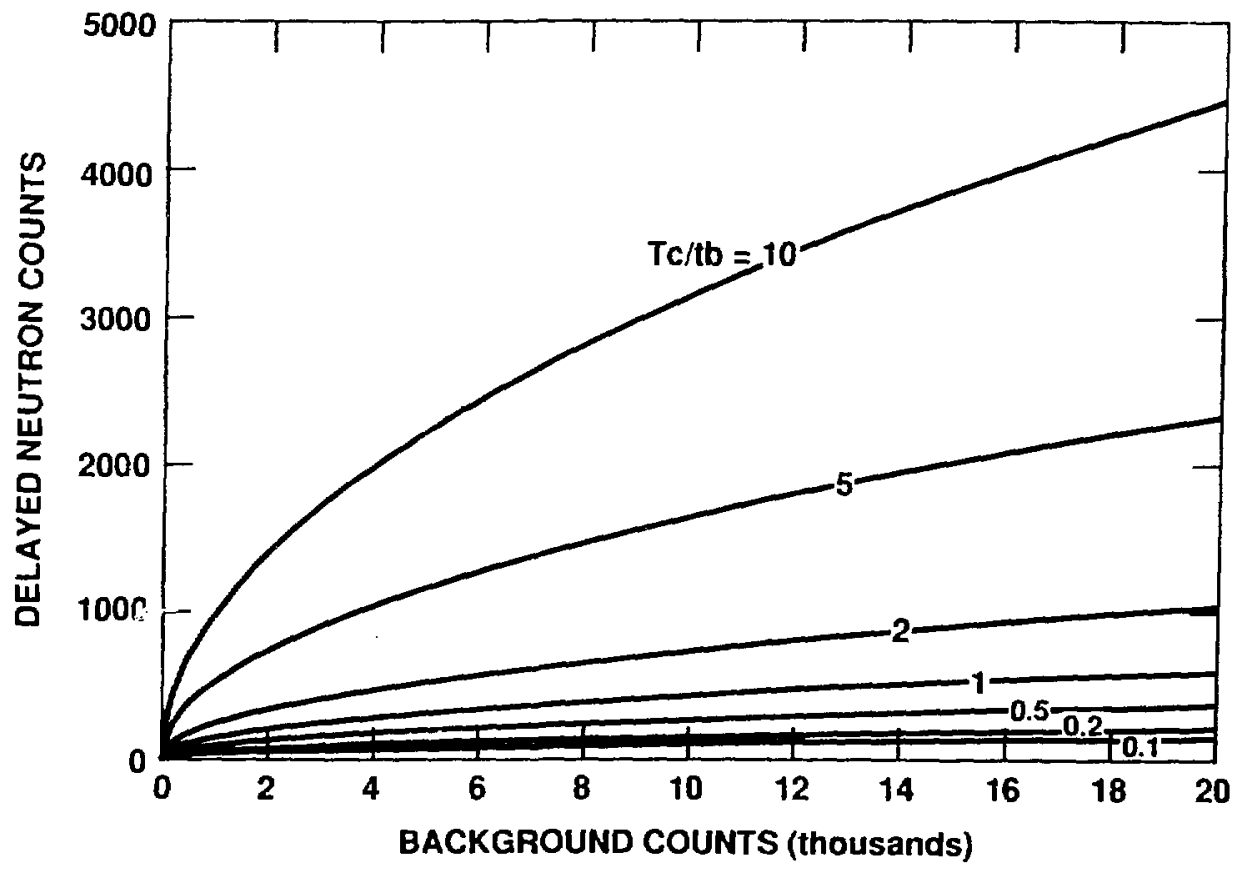

Fig. 17. The number of delayed-neutron counts during a time $T_{C}$ needed to have $D / \sigma_{D}=3$ is given as a function of the number of counts during a background time $t_{b}$. The different curves cover a range of $T_{C} / t_{b}$ values. If the shuffler is calibrated, the number of counts $D$ shown here can be converted into a mass of material which is the detection limit at $3 \sigma$ above background. 
become less important, but the source itself may be a large contributor to the background and thus $B_{b}$ can grow with the source size.

\section{Timing Optimization}

1. Maximum Delayed-Neutron Counts. The presence of background counts does not alter the conclusion in Sec. II.E that the maximum number of delayed-neutron counts is obtained by using $t_{i}=t_{c}$. The total number of counts is $D$ of Eq. (17) with the addition of $B$. However, maximizing $D$ is not likely to be the preferred goal of a measurement. The relative precision of a measurement is usually more important.

2. Minimum Relative Precision. A measure of merit commonly applied to an instrument is the size of its relative precision, which is $\sigma_{D} / D$ in Eq. (32). The parameters of an instrument that can most readily be adjusted to minimize the relative precision are $n, t_{i}, t_{c}, t_{b}$. It is assumed that $t_{f}$ and $t_{r}$ are fixed. If the size of the ${ }^{252} \mathrm{Cf}$ source is increased (with a corresponding increase in the background count rate) or the total assay time lengthened, the relative precision will improve.

Only a finite amount of time can be allotted to complete an assay, and the time usually includes both a background count time $i_{b}$ and an assay time $n \tau$. The total time $T$ is taken as a constant and is $t_{b}+n \tau$. With this relation, $t_{b}$ (or one of the other parameters) can be eliminated from Eq. (32).

To reveal all the times implicit in Eq. (32), replace the counts with rates and times. With $d$ as the average delayed-neutron count rate, $D=d T_{C}$ and $B_{t}=b t_{b}$. The background count rate $b$ is a constant, but $d$ is rather complex. With a larger number $n$ of short count times $t_{c}, d$ is relatively large because neutrons are being counted only during earlier portions of the exponential decays in Fig. 8. Furthermore, $d$ also depends on the nuclear properties of the isotopes involved, as shown in Eq. (17). For a particular set of conditions, the dependence of $d$ on $n$ is illustrated in Fig. 18.

The most straightforward way to find the optimum times is through a parameter search. The count rates $d$ and $b$ must be estimated. Values for $t_{f}$ and $t_{r}$ are selected and held constant. A set of values for $n \tau$ are selected; $t_{b}$ then follows as $T-n \tau$. The remaining parameters for the active assay $\left(n, t_{i}\right.$, and $t_{c}$ ) are then either varied individually or within certain constraints (such as $t_{i}=2 t_{c}$ ). Eventually a minimum $\sigma_{D} / D$ will be found.

Results of such searches are summarized in Fig. 19 for a case with $T=1000 \mathrm{~s}$ and $t_{i}=0.35 \tau$. Different values of $b$ were assumed and each led to a curve of optimum assay time $(n \tau)$ vs delayed-neutron count rate $(d)$.

\section{ISOTOPIC EFFECTS}

\section{A. Isotopes of a Single Element}

This section gives an outline of how a mixture of isotopes of the same element impacts the assay process with a shuffler; uranium is used as an illustration. The next section (VI.B) will discuss the case of a mixture of uranium and plutonium isotopes. 


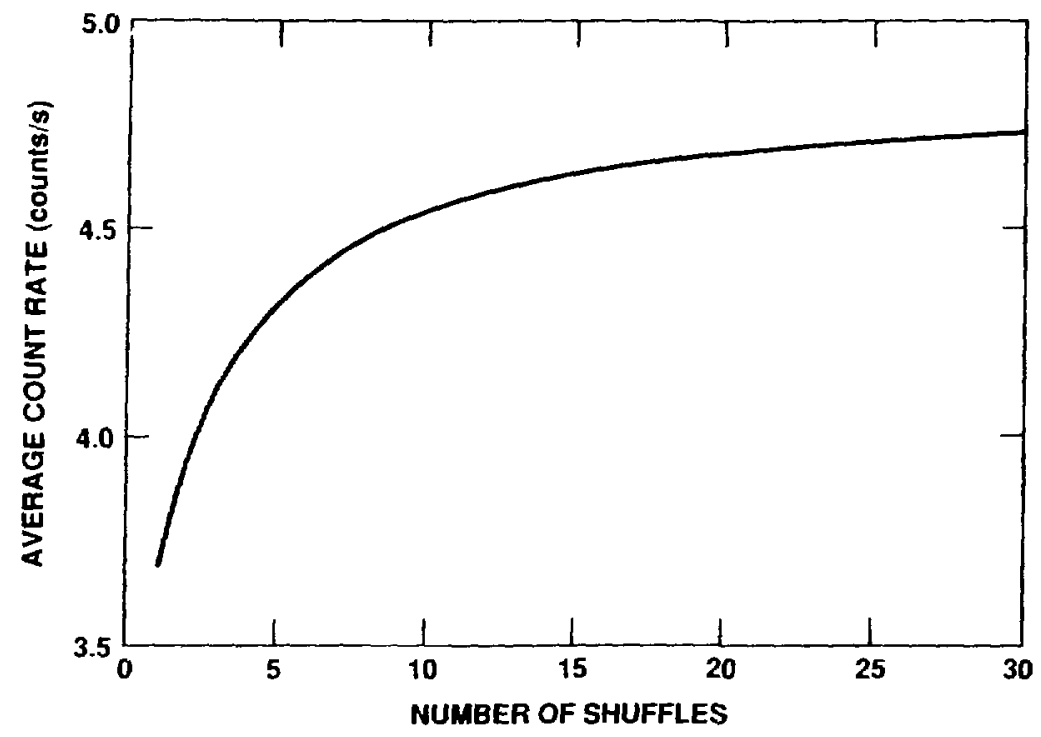

Fig. 18. The average count rate is the number of delayed neutrons counted over a sequence of shuffles divided by the total count time from all the shuffles. For the same conditions as in Fig. 16, the average count rate grows with the number of shuffles because the number rounted during a shuffle increases toward an equilibrium value (Figs. 13 and 15).

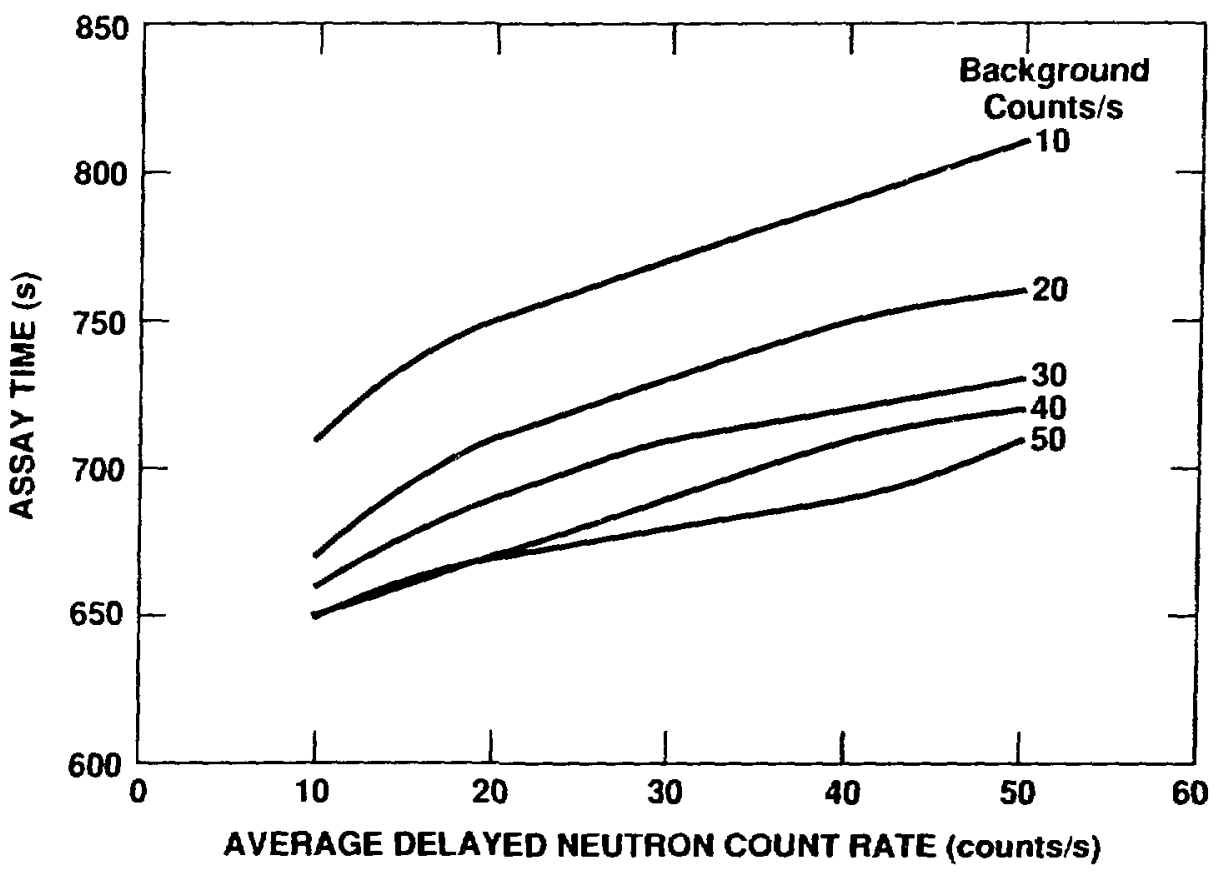

Fig. 19. If an assay is to be completed in a given time span, the time spent on a passive background count, the number of shuffles, and the times within a shuffle can be varied to minimize the relative counting precision.

This figure assumes that the total time available for a background count and an assay is $1000 \mathrm{~s}$. It is further assumed that $35 \%$ of the active interrogation portion of the assay time is spent counting delayed neutrons. Several different background count rates were used. As the averaged delayed-neutron count rate grows, it is better to spend less time on the background and more time on the assay. With higher background rates, more time should be spent on making a precise background measurement. 
1. The Problem. More than one uranium isotope is likely to be present in the material to be assayed and more than one isotope can produce significant numbers of delayed neutrons. If the isotopic composition of the material is different from that in the calibration standards, gross errors could result unless an accurate correction is applied.

The relative emission rates from equal amounts of precursors from three nuclides are shown in Fig. 20. These curves are based on the nuclear data in the Appendix $5-7,22,23$ and Eq. (5); the specific emission rate is defined as $\Sigma p_{j} \lambda_{j} / f$. The shapes of these curves vary among the isotopes, as seen in Fig. 21, where the emission rates are normalized to the start of the counting interval. This shape difference in principle could be used to obtain information on the isotopes present in the material being irradiated. However, it could be difficult to get enough counts to define the shape of the decay curve with sufficient precision and then to distinguish between (for example) $235 \mathrm{U}$ and a mixture of $238 \mathrm{U}$ and ${ }^{239} \mathrm{Pu}$. It will be assumed here that the isotopics are known and that only the area under the curve (the counts) is needed.

Assume a sample of uranium contains $50 \%{ }^{235} \mathrm{U}, 40 \%{ }^{236} \mathrm{U}$, and $10 \%$ other isotopes. The fission cross section of $236 \mathrm{U}$ is much lower than that of ${ }^{235} \mathrm{U}$ at all energies of interest here, but those fissions may still be about $20 \%$ of all the fissions (depending on the irradiation conditions). Furthermore, isotopes with higher atomic weights produce more delayed neutrons per fission (Fig. 5); in particular, each fast-neutron

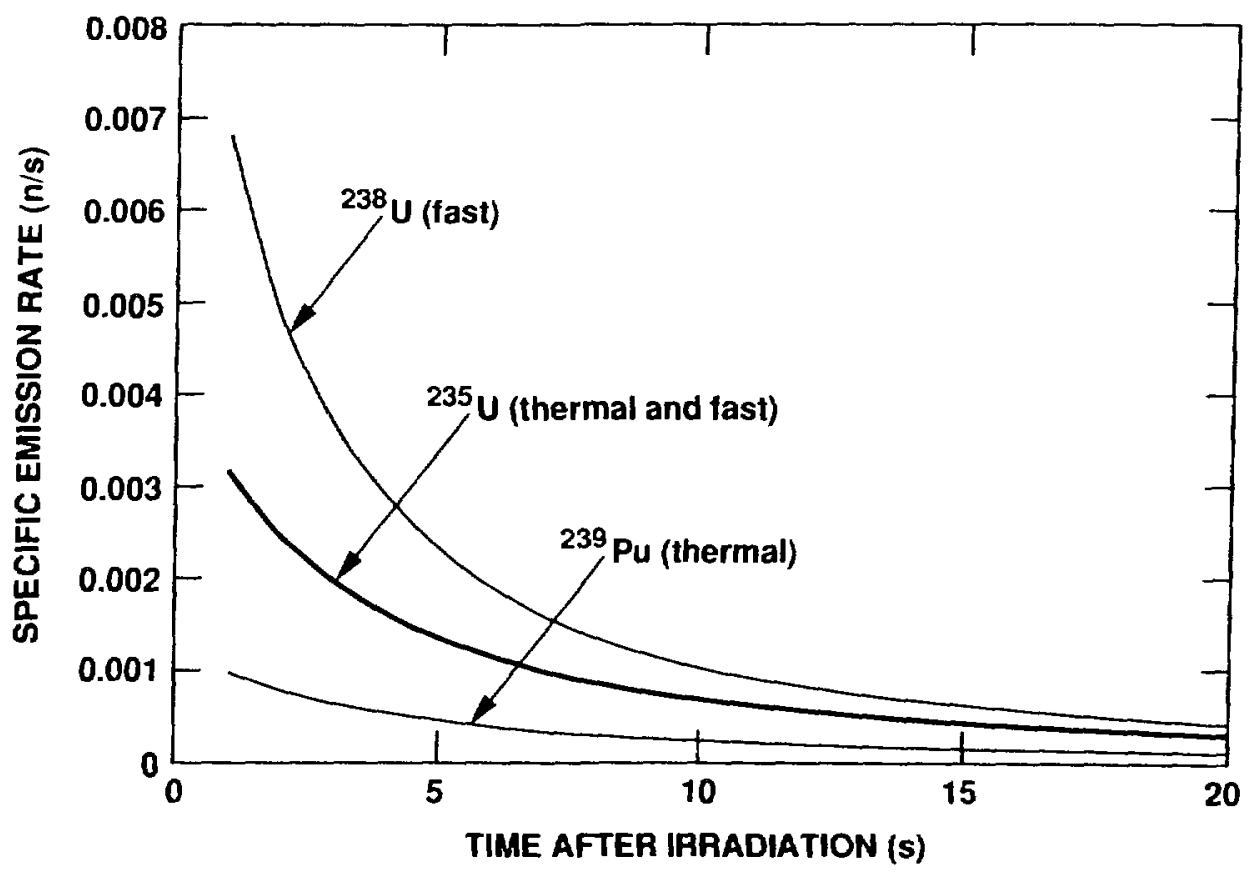

Fig. 20. After a sample of an isotope is irradiated for $10 \mathrm{~s}$, the delayed-neutron emission rate decays as shown here. Each curve is normalized to a single fission of an isotope. The fissions of ${ }^{235} \mathrm{U}$ by thermal neutrons produce only a slightly higher emission rate than do fast neutrons. 


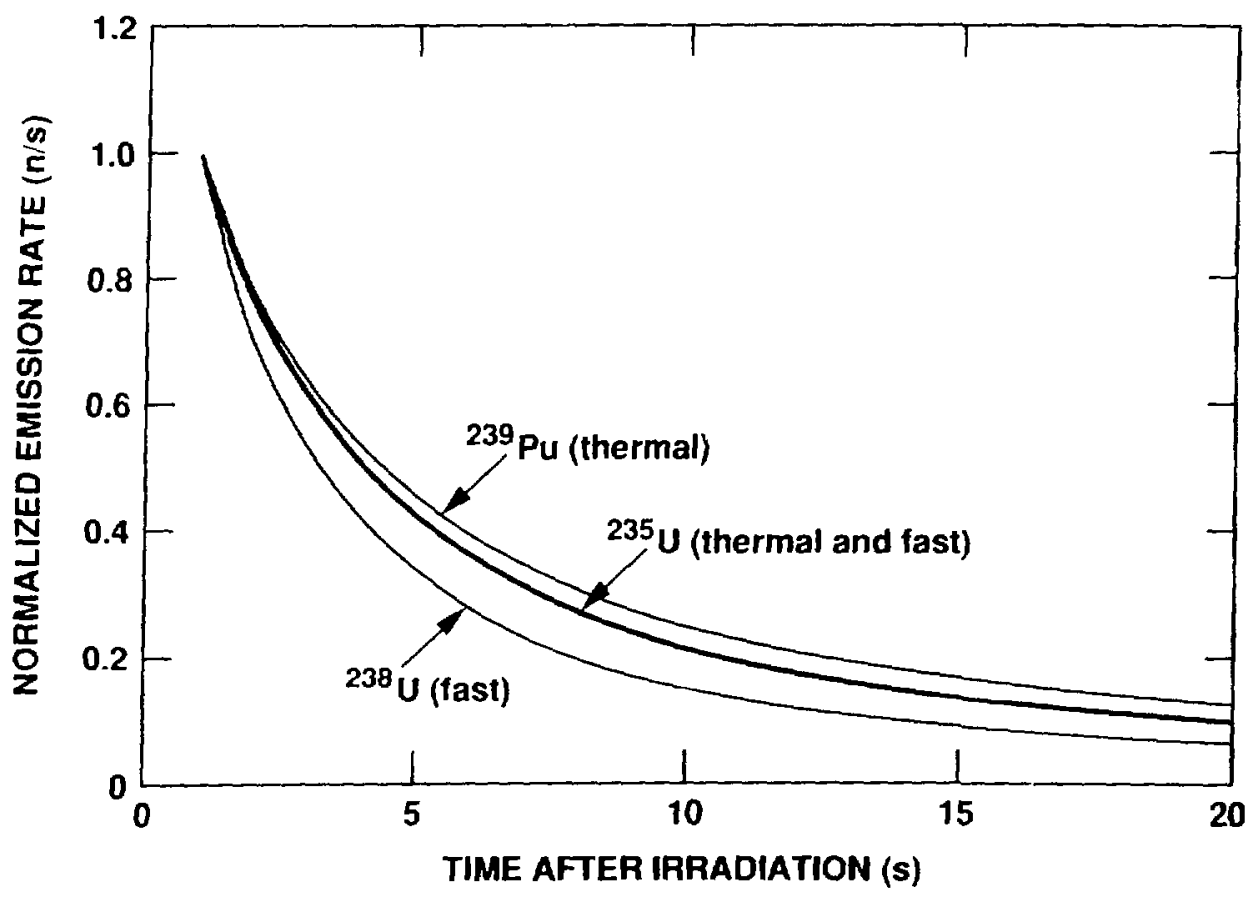

Fig. 21. The curves in Fig. 20 have been normalized to show their different shapes. The delayed neutron emission from ${ }^{238} \mathrm{U}$ decays the most quickly of these isotopes while those from ${ }^{239} \mathrm{Pu}$ are the longest lived.

fission of $236 \mathrm{U}$ produces $35 \%$ more delayed neutrons per fission than does a fastneutron fission of $235 \mathrm{U}$. Uranium-236 could be responsible for about one third of all the delayed-neutron counts. An assay that assumes all the delayed neutrons come from ${ }^{235} \mathrm{U}$ would be greatly in error. By using thermal neutrons to induce fissions, the signal from ${ }^{235} \mathrm{U}$ can be enhanced over that from 236U, but then inhomogeneities are poorly treated (Sec. III). A compromise must be carefully sought.

As another example, consider natural uranium with $0.7 \% 235 \mathrm{U}$ and $99.3 \% 238 \mathrm{U}$ (the very small amount of ${ }^{234} \mathrm{U}$ is ignored). Even if irradiated with thermal neutrons (for which the fission cross section in ${ }^{238} \mathrm{U}$ is essentially zero), there will be fissions in the $238 \mathrm{U}$ by fast neutrons released from $235 \mathrm{U}$ fissions. For high-density samples, it is possible that $1.5 \%$ of all fissions are in $238 \mathrm{U}$. The delayed-neutron production per fission in $238 \mathrm{U}$ is about 2.75 times greater than in ${ }^{235} \mathrm{U}$, so ${ }^{238} \mathrm{U}$ can account for more than $4 \%$ of all the delayed neutrons. For precise assays, this effect must be taken into account.

If a calculated correction is necessary (and it could be avoided with proper calibration standards), it can be based on detailed information about the delayed-neutron production by the isotopes when such information is available, or on more gross data 
that will give less accurate corrections. These two types of corrections are discussed in the following paragraphs.

2. Detailed Isotopic Correction. To make an isotopic correction to the delayed count rate, the isotopic composition must be known, along with the fractional delayedneutron yields, $\beta_{j}(i)$, for all precursor groups $j$ of isotopes $i$. The fission rates $f(i)=v(i) m(i) N_{A} \sigma_{f}(i) \phi / A(i)$ of the isotopes may be estimated with Monte Carlo calculations to take into account the geometry and neutron energy spectral effects. Fission rates need only be determined to within an overall constant (for example, the fission rate per unit californium source size), as will be seen just below. The mass of isotope $i$ is $m(i)=m($ total $) \cdot I(i)$, where $I(i)$ is the mass fraction of isotope $i$.

In an assay for the ${ }^{235} U$ content, a calibration curve between the mass and delayedneutron counts of that isotope would be used: $D(235)=f_{\text {calib }}[m(235)]$. The total uranium mass can then be found as $m(235) / I(235)$. However, the counts $D(235)$ from ${ }^{235} \mathrm{U}$ are only a fraction of the total counts.

The number of delayed-neutron counts from a single isotope has already been given in Eq. (17). By evaluating Eq. (17) for each isotope, a sum of the results gives the total delayed-neutron counts $D$ :

$$
D=\sum_{i} D(i)=\sum_{i} \sum_{j} D_{j}(i)
$$

Even if the calculated counts $D$ are only relative (for example, californium source size not specified), the measured counts can be fractured into isotopic components. In particular, the number of the measured counts that has come from $235 \mathrm{U}$ is $D(235)=D($ measured $) \cdot D^{\prime}(235) / D^{\prime}($ total $)$, where a prime indicates the relative nature of the calculated count. The calibration curve with this adjusted count $D(235)$ now yields $m(235)$ and the total uranium mass can be calculated as before by $m(235) / I(235)$.

3. Gross Isotopic Correction. To perform the calculations in the detailed isotopic correction, it is necessary to know the precursor group yields $\beta_{j}(i) v(i)$ and decay constants for all isotopes. However, these may not be known for all the isotopes involved. A correction can still be made with the total delayed-neutron yields $Y(i)=$ $v(i) \sigma \beta_{j}(i)$ from the different isotopes by ignoring the detailed nuclear and timing information required in Eq. (17).

The fission rate in isotope $i$ can be estimated by calculation (for example, Monte Carlo) and then multiplied by the total delayed-neutron yield. The fraction of the delayed neutrons originating from ${ }^{235} \mathrm{U}$ is then given by

$$
\frac{D^{\prime}(235)}{D^{\prime}(\text { total })}=\frac{f(235) \cdot Y(235)}{\sum_{i} f(i) \cdot Y(i)},
$$


ignoring the differences among delayed-neutron spectra and among countable fractions from different isotopes.

The ${ }^{235} \mathrm{U}$ contribution to the measured count rate is then

$$
D(235)=\frac{D(\text { measured }) \cdot D^{\prime}(235)}{D^{\prime}(\text { total })},
$$

and the $235 \mathrm{U}$ and mass can be found from the calibration curve.

Yields from ${ }^{234} \mathrm{U}$ and ${ }^{236} \mathrm{U}$ have not been measured. Estimates of them have been made by looking for systematic correlations 1,6 among yields and atomic numbers and masses, and from a detailed study of the precursors. ${ }^{8}$

The accuracy of this approach should be considered for each situation. For a mixture of $0.7 \% 235 \mathrm{U}$ and $99.3 \% 238 \mathrm{U}$, a Monte Carlo calculation for a particular geometry gave relative fission rates [all that is needed in Eq. (35)] of $f(235)=0.985$ and $f(238)=0.015$. The gross estimate of the ${ }^{235} \mathrm{U}$ fraction of the total count is 0.906 , whereas the detailed calculation using precursor groups gives 0.921 . The gross correction is in error by $1.5 \%$ in this case. With 3\%-enriched uranium, the error is 5 or $6 \%$.

For $1.5 \%{ }^{234} \mathrm{U}, 65 \%{ }^{235} \mathrm{U}, 22 \% 236 \mathrm{U}$, and $11.8 \%{ }^{238} \mathrm{U}$, a Monte Carlo calculation of the relative fission rates for a simple geometry yielded $0.00114,0.0769,0.00965$, and $1.878 \times 10^{-6}$, respectively. To do the detailed correction, calculated $236 \mathrm{U}$ parameters must be used. Ignoring the ${ }^{234} \mathrm{U}$, the detailed correction is 0.895 . The gross correction gives the $235 \mathrm{~L}$ fraction of the count as 0.888 .

Whether or not the gross correction should be used is dictated by the precursor group data available for the isotopes involved and how much accuracy is needed in the assay result.

\section{B. Mixture of Uranium and Plutonium Isotopes}

Uranium and plutonium isotopes are often found mixed together. Mixed-oxide fuel deliberately combines the two elements; spent light-water reactor fuel has a mixture of the two elements even though the fuel contained only uranium when fresh. The isotopes most often present that respond significantly to a shuffler's interrogation are ${ }^{235} \mathrm{U},{ }^{239} \mathrm{Pu}$, and ${ }^{241} \mathrm{Pu}$. Where there is ${ }^{241} \mathrm{Pu}$, there will also be ${ }^{241} \mathrm{Am}$; the delayed neutrons from ${ }^{241} \mathrm{Am}$ may not always be negligible.

The corrections discussed in the preceding section (VI.A) can be applied to each element of the mixture. Of more concern here is a procedure for determining the uranium and plutonium masses.

A single measurement cannot give the amounts of uranium and plutonium present, unless the ratios of their masses and isotopics are already known, which is not likely. 
To assay this mixture of elements, three measurements are probably needed (it is assumed that the uranium isotopics are already sufficiently understood). The three measurements are

- a high-resolution gamma-ray spectral determination of the plutonium isotopics,

- a passive neutron count with the shuffler to find the plutonium masses through counting coincidence neutrons, and

- an active shuffler interrogation to measure the uranium mass as the difference between the net fissile mass and the plutonium mass. ${ }^{24}$

The gamma-ray method for obtaining the plutonium isotopics will not be discussed here. References 25-27 provide information on this topic.

Plutonium assays with passive coincidence measurements of neutrons emitted by the spontaneous fission of the isotopes of plutonium with even atomic weights (especially with mixed-oxide materials) are described in Refs. 24, 28, and 29. Basically a passive count gives the amount of plutonium and the amounts of ${ }^{239} \mathrm{Pu}$ and ${ }^{241} \mathrm{Pu}$ follow from the plutonium isotopics.

The average delayed-neutron count rate from the shuffler measurement depends on the masses and relative intrinsic responses of the isotopes present. For the three isotopes assumed here,

$$
d=a_{235} m(235)+a_{239} m(239)+a_{241} m(241),
$$

where the $a_{i}$ is a coefficient for isotope $i$ that depends on the fission rates and delayedneutron yield, and $m(i)$ is the mass of isotope $i$. The coefficients can be determined from measurements with standards and the masses $m(239)$ and $m(241)$ are found from the passive neutron coincidence count. The only unknown remaining in the equation is $m(235)$, the mass of $235 \mathrm{U}$.

\section{INHOMOGENEOUS MATERIALS}

If the material being assayed is known to be homogeneous (to whatever accuracy is sufficient), relatively relaxed procedures can be followed. Thermal neutrons can be used for an enhanced count rate even though they do not penetrate the material; the source of irradiating neutrons can be placed anywhere relative to the material as long as the position is reproducible; the detection responses to different regions of the materials need not be uniform. 
If inhomogeneities in the material are possible, the procedures used in making an assay can greatly affect the accuracy of the assay. An accurate assay depends on counting a number of delayed neutrons from each region of the material that is proportional to the fissile material's density in that region. Both the production of delayedneutron precursors and the sensitivity of the detectors to different regions of the material are involved.

Neutrons must penetrate the volume of an inhomogeneous object to seek lumps, voids, or less drastic density changes, so high-energy neutrons are necessary. Thermal neutron absorbers (such as cadmium) should surround the material to block the entrance of thermal neutrons into the material. A compromise on the energy spectrum's hardness may be required to reduce fissions in isotopes such as ${ }^{238} \mathrm{U}$ when the amount of $235 \mathrm{U}$ is sought, for example.

The rest of this section deals with the uniform irradiation of the material and flattening the detection response profile to delayed neutrons released throughout the material.

\section{A. Uniform Irradiation}

The source of irradiating neutrons is very likely to be much smaller than the material being assayed. A capsule of ${ }^{252} \mathrm{Cf}$ or a D-T generator is essentially a point source of neutrons compared to the cans, boxes, and barrels to be assayed (although neutron reflectors can be used to somewhat increase the apparent size). Furthermore, it is usually necessary to place the source to one side of the container rather than in some position for symmetric irradiation.

A simple strategy is to keep the source far enough from the container to yield a uniform flux of neutrons through the container. However, this requires a very strong source and a much larger overall size and cost for the shuffler.

The source strength and shuffler size can be minimized by keeping the source near the container. An approximately uniform irradiation of the container is generated by rotating the container and moving the source parallel to the axis of rotation ("scanning"). A scanning motion with a ${ }^{252} \mathrm{Cf}$ source on a motor driven cable can be readily performed, whereas this is impractical with pneumatic drives or D-T generators.

A scanning procedure introduces the complication of a time-dependent flux in the material. The goal is not simply to produce a uniform distribution of fissions throughout the material, but to have a uniform distribution of delayed-neutron precursors at the end of the scan. These are different subjects because the precursors are created and decay throughout the whole scan. By using scan times (about 5 s) short relative to important precursor decay half-lives (5-25 s), uniform irradiation can be sought with only slight asymmetries in the precursor distribution.

A complication that will not be pursued here is that the matrix in the container can interfere with a uniform irradiation of the fissile isotopes. If a portion of the fissile material is surrounded by hydrogenous materials, for example, it will experience a different flux than material surrounded by metal. Devising an appropriate scanning 
procedure requires knowing the matrix distribution and its effects. Gamma-ray radiography is one technique for estimating matrix densities, but the problem has not yet been seriously approached for shufflers.

Several parameters can be adjusted to improve the uniformity of the irradiation.

- The ratio of the scan and rotation periods should be chosen so that the fluence across the surface of the container is as uniform as possible. A very rapid and repetitive motion of the source would approximate a line source, but could place unwanted (and perhaps unnecessary) stresses on the motion controller. One strategy is to choose periods that form an irreducible ratio $(4 / 3$, for example) and whose product is equal to the scan time $(4 \cdot 3=12$, for example).

- The length of the scan relative to the length of the container can be adjusted. By overscanning (going beyond the ends of the container) the ends of the material are given additional neutrons that can help balance their irradiation relative to the container's center.

- At the overscan positions the source can be halted for a brief time to further increase the irradiation of the ends.

- The speed of the source during the scans can be greater near the center than near the ends.

A mathematical investigation can be made based on Eq. (1). The fission neutron production rate $f$ in Eq. (1) is now taken to be a function of the positions of the source $x_{s}(t)$ and the material $x_{m}: f\left[x_{s}(t), x_{m}\right]$. The source position is a function of time, so the overall creation and decay of precursors in the material is quite complex. Equation (1) can be evaluated for different circumstances and the most favorable scheme identified. Solutions may be needed at only a few representative material positions, $x_{m}$, in order to examine the relative sizes of the precursor populations along the axis of the material.

\section{B. Detection Response Profile}

Even if the irradiation of the material has been uniform and the production of delayed neutrons is proportional to the amount of uranium throughout the material, the assay of an inhomogeneous material will be inaccurate if the detection process is not equally sensitive to neutrons released at different locations and at different energies.

Regularly spaced detector tubes and rotation of the material will help make the detection process more independent of the source location.

The detection sensitivity axial profile can be flattened by using detector tubes that are much longer than the material. A practical limit to the length will usually introduce reduced sensitivity to emissions from the material's ends. 
A useful estimate of the shape of the sensitivity profile can be made by simply calculating the solid angles subtended by the detector from points on the axis of the material. Even though the material is not a line source, this calculation has been sufficiently accurate in many cases. The required length of the detector tubes can then be estimated from the maximum allowed reduction in sensitivity from the ends relative to the center.

Let the lengths of the detector tubes be $L$ and let the tubes form a ring of radius $R$. Assume the source is on the center line of the cylinder formed by the ring of tubes and is a distance $z$ from the bottom of the tubes. The solid angle subtended by the ring of tubes is

$$
\Omega=4 \pi-2 \pi\left\{\frac{\sqrt{R^{2}+(L-z)^{2}}-(L-z)}{\sqrt{R^{2}+(L-z)^{2}}}\right\}-2 \pi\left\{\frac{\sqrt{R^{2}+z^{2}}-z}{\sqrt{R^{2}+z^{2}}}\right\} .
$$

The decrease in the sensitivity at the ends can be quickly found by comparing $\Omega$ at an end $(z=0$ or $L)$ and at the center $(z=L / 2)$.

The response can also be flattened by making the center region of the detectors less sensitive. Properly sized and spaced strips of cadmium can be wrapped around the detector tubes to reduce the thermal neutron flux entering the center region.

The delayed neutrons have a spectrum of energies, 8 which is further modified by their transport out of the container and into the banks of detectors. By placing detector tubes at different depths inside the polyethylene, the count rate can be made nearly independent of the energy of neutrons incident on the detector banks. The best positions of the tubes depend on the specific geometry of the neighboring portions of the shuffler. Ratios of counts from the different tubes may also help correct for the matrix effects within the material being assayed.

\section{FLOWING-LIQUID SHUFFLER}

\section{A. Introduction}

A shuffler can monitor the fissile content in a flowing stream. The timing of the assay should take into account the highest flow rate; if the liquid completely changes within the assay chamber during the irradiation phase, there may be no delayed neutrons to count later. It is assumed here that the flow rate is low enough to allow the shuffler to irradiate and count a portion of the liquid at least a few times before it leaves the assay chamber.

The assay chamber contains an influx of new, unirradiated material and an efflux of material that has been irradiated by some shuffles of the ${ }^{252} \mathrm{Cf}$. After a certain number of consecutive shuffles following start-up, a steady-state condition is reached within the assay chamber. 
When the flow rate may vary, an adjustment for the flow rate is needed. A single calibration curve for the concentration of fissile material in the liquid can then serve all flow rates.

The fraction of delayed-neutron counts lost is not simply the fraction of the liquid that flows out during a shuffle because the material being lost has been in the assay region the longest and is a more prolific producer of delayed neutrons per unit volume than the material near the inlet end. The fraction lost depends on the assay region's geometry, the flow rate, and the delayeci-neutron properties of the precursor groups.

\section{B. Flow-Rate Adjustment}

To be able to use a single calibration curve for all flow rates, we must adjust the measured count rates to a standard flow rate. If one flow rate is much more common than others, the size of the adjustment will be minimized by adjusting to that standard flow rate.

The delayed-neutron counts at the standard and other flow rates can be measured using a solution as similar as possible to the actual solutions to be monitored. If $F_{\text {std }}$ is the standard flow rate, the counts with it are $D\left(F_{s t d}\right)$. For another flow rate $F$, the measured counts are $D(F)$. The flow rate correction factor is then $C F_{f l o w}(F)=D\left(F_{s t d}\right) / D(F)$.

An assay's count, $D$, at the flow rate, $F$, is converted to the adjusted count, $D_{\text {flow, }}$ for the flow rate, $F_{\text {std }}$,

$$
D_{\text {flow }}=D \cdot C F_{\text {flow }}(F)
$$

An example of the magnitude of the correction factor $D\left(F_{s t d}\right) / D(F)$ is given in Fig. 22 for nitric acid with $0.48 \mathrm{~g} / \mathrm{L}$ of $235 \mathrm{U}$. The assay chamber was a cylindrical annulus $6 \mathrm{in}$. high, with an inner radius of $1.5 \mathrm{in}$. and an outer radius of $3 \mathrm{in}$. Unless the flow rate is quite steady at the value used during calibration, large errors can be introduced if an adjustment such as in Eq. (37) is not applied.

\section{DATA ANALYSIS SUMMARY}

The raw data collected during an assay consist of times and neutron counts. This chapter gives a stepwise procedure for converting these data into an assay result. This is likely to involve a computer code and the analysis that follows has that in mind. 


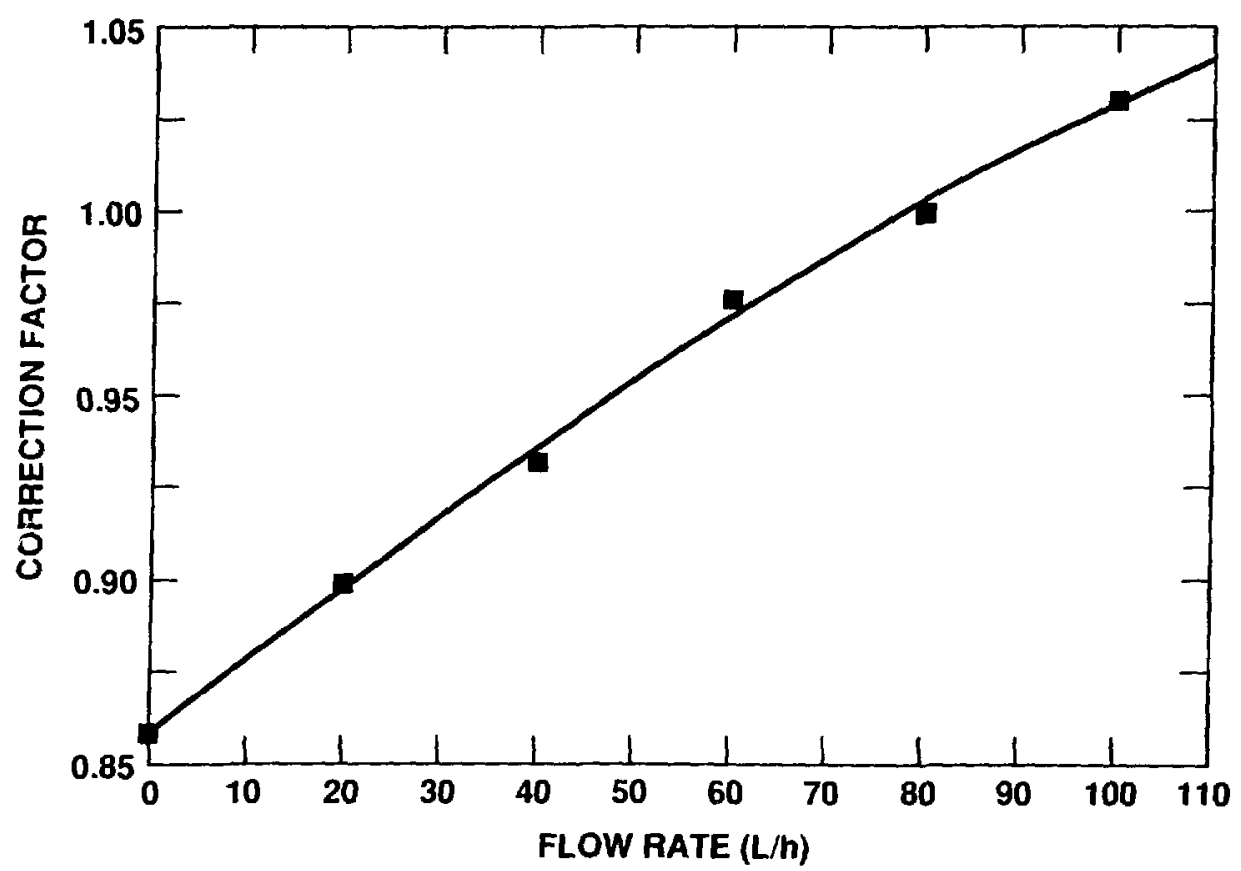

Fig. 22. Delayed neutron count rates from a liquid were measured at different flow rates to generate this flow-rate-correction-factor curve for a particular assay geometry. A nitric acid solution $\left(0.48 \mathrm{~g} / \mathrm{L}\right.$ of $\left.{ }^{235} \mathrm{U}\right)$ was driven through an assay chamber and the flow rate monitored. The standard flow rate was taken as $\mathbf{8 0}$ $\mathrm{L} / \mathrm{h}$ and the adjustments were made to that flow rate. For example, a static solution needs to have its count rate multiplied by 0.86 to use a calibration curve developed for a flow rate of $80 \mathrm{~L} / \mathrm{h}$.

\section{A. Assay Calculation}

There are several portions of the analysis procedure, not all of which may apply to a given case:

- subtract the background counts;

- adjust for the decay of the ${ }^{252} \mathrm{Cf}$ source;

- adjust for the flow rate, if any, of liquid passing through the monitor;

- adjust for variations that affect the flux of the irradiating neutrons;

- adjust for the minor variations in the timing of the segments of an assay;

- make a normalization correction, if necessary;

- determine the assay value from the calibration;

- make a bias correction, if necessary. 
1. Start-up Calculations and Initializations. Upon the start-up of the computer code, some items can be calculated that depend only on the parameters, not the measured data.

The calculation of the correction for irregular times (Sec. IV.B) can begin before any data taking. Equation (17) can be evaluated using nominal values and setting $\varepsilon f=1$. [Equation (28) will be used with actual times and with $\varepsilon f=1$ also; the correction factor in Eq. (29) shows that the actual value of of is immaterial.]

Use the nominal times $t_{f_{n}}, t_{n_{n}}, t_{r_{n}}$, and $t_{c_{n}}$, the nominal number of shuffles per assay $n_{n}$, and the nuclear data information $\beta_{j}$ and $\lambda_{j}$ to calculate $D_{n}^{\prime}$ from Eq. (17).

$$
\begin{aligned}
\tau_{n}= & t_{f_{n}}+t_{i_{n}}+t_{r_{n}}+t_{c_{n}} . \\
D_{n}^{\prime}= & \sum_{j=1}^{6}\left(\frac{\beta_{j}}{\lambda_{j}}\right)\left[1-\exp \left(-\lambda_{j} t_{i_{n}}\right)\right]\left[\exp \left(-\lambda_{j} t_{r_{n}}\right)\right]\left[1-\exp \left(-\lambda_{j} t_{c_{n}}\right)\right] \\
& \cdot\left[\frac{n_{n}-\left(n_{n}+1\right) \exp \left(-\lambda_{j} t_{n}\right)+\exp \left[-\left(n_{n}+1\right) \lambda_{j} \tau_{n}\right]}{\left[1-\exp \left(-\lambda_{j} \tau_{n}\right)\right]^{2}}\right]
\end{aligned}
$$

Some other cycle correction items need to be initialized. The calculated relative number of counts for the measured times will have contributions after every shuffle, so it is initially set to zero.

$$
D^{\prime}=0
$$

Some items called $P_{j}^{\prime}$ will also be used in evaluating Eq. (28) and they are also set to zero at this time.

$$
P_{j}^{\prime}=0, \quad j=1,2, \ldots, 6
$$

The correction factor for the decay of the ${ }^{252} \mathrm{Cf}$ source can be calculated at the start of an assay, using the decay constant $\lambda$ for ${ }^{252} \mathrm{Cf}$.

$$
d d=\text { number of }{ }^{252} \mathrm{Cf} \text { decay days since the reference date } .
$$




$$
C F_{C f}=e^{\lambda(d d)}
$$

If the fissile material is a flowing liquid, the correction factor for the flow rate can also be calculated at this time.

$$
C F_{\text {flow }}(F)=\frac{D\left(F_{\text {std }}\right)}{D(F)}
$$

2. Electronics Checks. If two banks of delayed-neutron detectors are being used, the ratio of their counts can be compared to a reference value and its acceptance limit stored in a parameter file. If the ratio is not within the limit, appropriate action can be taken (for example, abort the assay; notify an operator; retry the assay). A similar check can be made on the ratio of counts from flux monitor tubes.

These comparisons can be made after every shuffle and again at the end of an assay.

3. Intermediate Values after a Shuffle. After a shuffle, the raw data of interest are the four times, $t_{f s}, t_{i s}, t_{r s}$ and $t_{c s}$ plus the counts in the delayed-neutron channel $C_{D s}$ and the flux monitor channel $C_{M s}$. These counts still include background contributions.

Certain sums over $s$ are needed for the assay calculation later and can be updated after every shuffle.

$$
\begin{aligned}
& T_{l}=\sum_{s} t_{i_{s}} \\
& T_{C}=\sum_{s} t_{c_{s}} \\
& C_{D}=\sum_{s} C_{D_{s}} \\
& C_{M}=\sum_{s} C_{M_{s}} .
\end{aligned}
$$

The cycle correction factor can be partially calculated after every shuffle using these equations.

$$
P_{j s}=\left(\frac{\beta_{j}}{\lambda_{j}}\right)\left[1-\exp \left(-\lambda_{j} t_{i_{s}}\right)\right]\left[\exp \left(-\lambda_{j} t_{r_{s}}\right)\right]+P_{j, s-1}^{\prime} \cdot \exp \left[-\lambda_{j}\left(t_{f_{s}}+t_{i_{s}}+t_{r_{s}}\right)\right]
$$




$$
\begin{aligned}
D_{j s}= & P_{j s}\left[1-\exp \left(-\lambda_{j} t_{c_{s}}\right)\right] \\
D^{\prime}= & D^{\prime}+\sum_{j} D_{j s} . \\
P^{\prime}{ }_{j s}= & P^{\prime}{ }_{j, s-1}\left\{\exp \left[-\lambda_{j}\left(t_{f_{s}}+t_{i_{s}}+t_{c_{s}}+t_{r_{s}}\right)\right]\right\} \\
& +\left(\frac{\beta_{\mathrm{i}}}{\lambda_{\mathrm{j}}}\right)\left\{\exp \left[-\lambda_{j}\left(t_{r_{s}}+t_{c_{s}}\right)\right]\right\} \cdot\left[1-\exp \left(-\lambda_{j} t_{c_{s}}\right)\right] .
\end{aligned}
$$

In Eq. (46), the $P^{\prime}$ are from the previous shuffle (or zero if $s=1$ ), while in Eq. (49) the new $P^{\prime} j$ are calculated for use after the next shuffle. Equation (48) is not an algebraic equation but rather the usual computer code statement for adding new terms into an existing sum, which in this case is $D^{\prime}$. This set of equations is equivalent to Eqs. (24)(28) in Sec. IV.A.

4. Assay Count Rates. When all $n$ shuffles are completed, the assay value can be calculated. The first step is to find the count rates of delayed neutrons, $d$, and neutrons counted by the flux monitor, $m$ (including backgrounds), using the values from Eq. (44).

$$
\begin{aligned}
& d=\frac{C_{D}}{T_{C}} . \\
& m=\left(\frac{C_{M}}{T_{I}}\right) \exp \left(\frac{\delta C_{M}}{T_{I}}\right) .
\end{aligned}
$$

A deadtime correction factor with a deadtime $\delta$ is included in the flux monitor rate because of the high count rates expected and because the count rates will change as the ${ }^{252} \mathrm{Cf}$ source decays. The deadtime needs to be measured for each instrument, but it is typically on the order of a microsecond.

It is preferable to work with count rates rather than counts to eliminate the dependence on count times.

5. Background and ${ }^{252} \mathrm{Cf}$ Decay Corrections. The backgrounds may have contributions from the ${ }^{252} \mathrm{Cf}$ source and sources outside the shuffler. The outside background 
rate may or may not be relatively constant, but the rate from the ${ }^{252} \mathrm{Cf}$ source will decay with time.

If delayed-neutron and flux-monitor background count rates $\left(b\right.$ and $\left.b_{M}\right)$ are taken just before or after an assay count, they can be subtracted from the delayed-neutron and flux-monitor count rates and then corrected for the decay of the ${ }^{252} \mathrm{Cf}$ source.

$$
\begin{aligned}
& d_{1}=(d-b) \cdot C F_{C f} . \\
& m_{1}=\left(m-b_{M}\right) \cdot C F_{C f} .
\end{aligned}
$$

When taking assays continuously, it may not be practical to take a background with every assay. For example, while monitoring the fissile concentration in a flowing liquid stream to avoid a criticality condition, it would be dangerous to spend half the time taking backgrounds.

When the background originates partially from the ${ }^{252} \mathrm{Cf}$ and partially from the outside, the appropriate expression for an assumed background rate as a function of time is more complex and will not be considered further here.

6. Flow-Rate Correction. If the material being assayed is a flowing liquid, the flow rate is not likely to always be the same as the rate during calibration. If the shuffler's computer is given the flow rate upon demand, the information can be used to correct for different flow rates. A single calibration curve can then still be used for all situations.

$$
d_{2}=d_{1} \cdot C F_{\text {flow }}[F,\{g\}]
$$

where $F$ is the flow rate and $\{g\}$ are parameters to be determined for the situation at hand. The correction factor shown in Fig. 22 is well described by a second-order polynomial: $C F_{\text {flow }}[F,\{g\}]=g_{2} F^{2}+g_{1} F+g 0$. The parameters can be chosen to make the correction factor 1 at the nominal flow rate.

The flux monitor response during irradiation is not likely to be affected by the flow rate because the large count rate from the ${ }^{252} \mathrm{Cf}$ should overwhelm the small count rate from the material being assayed.

7. Flux-Monitor Correction. If flux monitors are used, the measured count rate $m_{1}$ can be compared to an expected or nominal rate $m_{n}$. If the amount of moderator in the material is unusually high, for example, the measured rate $m_{1}$ may be unusually large and the calibration curve to be applied later is inappropriate. The best way to use the $m_{1}$ and $m_{n}$ values may vary according to the material under study, so this equation can only be an example of how a correction may be applied. 


$$
d_{3}=d_{2} \cdot C F_{f l u x}\left(m_{1},\{q\}\right)=d_{2} \cdot\left(\frac{m_{n}}{m_{1}}\right)^{q}
$$

In general, there may be more than one parameter, but in this example only one is used, so it is called $q$ instead of $q_{1}$. This sort of correction with $q=1$ has often been used, but different values of $q$ have been suggested. ${ }^{30,31}$ No guidelines will be given here in selecting $q$.

It may be best to apply this correction factor only when it exceeds a certain threshold. The threshold might be three or four times the standard deviation of $\left(m_{n} / m_{1}\right)^{q}$. Without a threshold, the fluctuations in the correction factor will introduce fluctuations in $d_{3}$ that could do more harm than good. With a threshold, a correction is made only if it is statistically significant.

A ratio of counts from different rings of detectors has been used instead of a ratio of counts from flux monitors. The expression in Eq. (54) might still be used, with $m_{n}$ and $m_{1}$ replaced by counts in the two rings. It might be necessary to use a different expression entirely. The general subject of how to correct for flux changes is still open.

8. Cycle Correction. With $D^{\prime}$ completed after the last shuffle, the count rate can be adjusted for deviations from the nominal assay conditions (timings, number of shuffles), as suggested in Eq. (29). The $D_{n}^{\prime}$ and $D^{\prime}$ needed here are calculated with Eqs. (38) and (47).

$$
d_{4}=d_{3} \cdot C F_{\text {cycle }}\left(D^{\prime}\right)=d_{3} \cdot\left(\frac{D_{n}^{\prime}}{D^{\prime}}\right)
$$

A threshold can also be used on this correction factor to again avoid generating unnecessary fluctuations.

This factor is typically very close to 1 and with a stable mechanical system will have very small variations. If the correction factor should become large, it could indicate a malfunction of the equipment.

9. Normalization. If there is reason to believe a normalization is required, it should be applied at this point. Normalization can be needed to correct for electronics drift or failure of a detector tube, for example. It is not a correction that should be needed routinely and can be eliminated by repairing any equipment problems or recalibrating. 
The normalization factor is typically generated by measuring a reference standard and comparing the counts or count rate $D^{\prime \prime}$ to a nominal value $D_{n}{ }^{\prime \prime}$.

$$
d_{5}=d_{4} \cdot C F_{n o r m}\left(D^{\prime}\right)=d_{4} \cdot\left(\frac{D_{n}^{n}}{D^{n}}\right) .
$$

A threshold for applying this correction is also appropriate in this case.

10. Assay Value. The assay value $A$ (for example, mass or concentration) is interpolated from a calibration curve, which is only shown formally here with a set of parameters $\{p\}$.

$$
d_{5}=f_{\text {calib }}(\mathrm{A},\{p\})
$$

The desired value of $A$ can be found regardless of the complexity of the function by using a general numerical technique described later.

11. Bias Correction. Despite the best of efforts with the above analysis, a known bias in the results may exist, so a bias-corrected assay, $A_{b c}$, may be needed. This is indicated formally here as a function of $A$ with parameters $\{b\}$.

$$
A_{b c}=f_{b i a s}(A,(b)) .
$$

The form of the function is highly dependent on the situation at hand and no typical form can be given. The nature of a bias correction can be as difficult to determine as the source of the bias.

\section{B. Assay Uncertainties}

1. Random, Systematic, and Total. An assay result has three types of uncertainties: random $\left(\sigma_{r}\right)$, systematic $\left(\sigma_{s}\right)$, and total $\left(\sigma_{A}\right)$. They are related through their variances.

$$
\sigma_{A}^{2}=\sigma_{r}^{2}+\sigma_{s}^{2}
$$

Random uncertainties arise from fluctuations that are inherent and unavoidable because of the nature of the items being measured. The random uncertainty is often called the precision of the instrument. 
Systematic uncertainties arise during the corrections to the data and from interpretations given to the data. The systematic uncertainty is sometimes called the bias of the instrument, although this term will not be used in this context here. Variations in the samples' geometries and other uncontrolled features add to the systematic uncertainty but cannot be included in the calculated systematic uncertainty.

For a shuffler, there are four sources of random uncertainties: measured count rates of delayed neutrons and their backgrounds, measured count rates of flux monitors and their backgrounds, the correction for the decay of ${ }^{252} \mathrm{Cf}$, and measured times in the cycle correction.

The sources of systematic uncertainties in the preceding analysis are these: the flow rate correction, the normalization process, and the calibration curve.

The correction for the ${ }^{252} \mathrm{Cf}$ decay is taken here as having random uncertainties. The uncertainty in the decay constant by itself would lead to a systematic uncertainty, as would the number of days of decay if calculated by a systematically incorrect procedure. However, there could be random (or at least hard-to-predict) variations in the number of days of decay if the algorithm uses an integer number of days (ignoring hours and minutes). Whether this correction introduces random or systematic uncertainties is not important in practice because the uncertainty is very small and negligible compared to others.

2. The Total Uncertainty. The development of expressions for these uncertainties starts with the calibration relation between the assay value $A$ and the corrected count rate $d_{5}$. General expressions will be given along with specific examples that assume certain relations; if other relations apply, the general expressions show how to use them.

The general relationship between $d_{5}$ and $A$ is

$$
\begin{aligned}
d_{5} & =(d-b) \cdot C F_{C f} \cdot C F_{f l o w} \cdot C F_{f l u x} \cdot C F_{\text {cycle }} \cdot C F_{\text {norm }} \\
& =f_{\text {calib }}(A,\{p\}) .
\end{aligned}
$$

Using the examples of the correction factors from the preceding sections and a second-order polynomial for the calibration function, this relationship becomes

$$
\begin{aligned}
d_{5} & =(d-b) \cdot e^{\lambda \cdot d d}\left(g_{2} F^{2}+g_{1} F+g_{0}\right) \cdot\left(\frac{m_{n}}{m_{1}}\right)^{q} \cdot\left(\frac{D_{n}^{\prime}}{D^{\prime}}\right) \cdot\left(\frac{D_{n}^{\prime \prime}}{D^{\prime \prime}}\right) \\
& =p_{2} A^{2}+p_{1} A+p_{0} .
\end{aligned}
$$


With this particular calibration function, the quadratic formula can be used to find $A$, but a numerical method can be used to find $A$ regardless of the form of the function (Sec. VIII.C.1).

The variance (square of the uncertainty) of $A$ is

$$
\sigma_{A}^{2}=\left(\frac{\partial A}{\partial t_{5}}\right)^{2} \sigma_{d 5}^{2}+\sum_{i} \sum_{j}\left(\frac{\partial A}{\partial p_{i}}\right)\left(\frac{\partial A}{\partial p_{j}}\right) \sigma_{p_{i} p_{j}}
$$

The $\sigma_{m}$ are covariances if $m \neq n$ and variances $\left(\sigma_{m}^{2}\right)$ if $m=n$.

The partial derivatives can be expanded into simple expressions. The two derivatives of $A$ are simple and are evaluated first, followed by the more complex variance of $d_{5}$. The variances and covariances represented by $\sigma_{p_{i} p_{j}}$ are generated by the curvefitted procedure used in finding the parameters $\{p\}$.

In general,

$$
\left(\frac{\partial A}{\partial d_{5}}\right)=\frac{1}{\left(\frac{\partial d_{5}}{\partial A}\right)}=\left(\frac{1}{\frac{\partial f_{\text {calib }}}{\partial A}}\right)
$$

whereas for the particular calibration function of Eq. (62),

$$
\left(\frac{\partial A}{\partial d_{5}}\right)=\frac{1}{\left(2 p_{2} A+p_{1}\right)} .
$$

In the rest of the equations, the general expression will be immediately followed by a specialized expression using the appropriate example function given earlier.

$$
\begin{aligned}
\left(\frac{\partial A}{\partial p_{i}}\right) & =\left(\frac{\partial A}{\partial d_{5}}\right)\left(\frac{\partial d_{5}}{\partial p_{i}}\right)=\frac{\left(\frac{\partial f_{\text {calib }}}{\partial p_{i}}\right)}{\left(\frac{\partial f_{\text {calib }}}{\partial A}\right)} \\
& =\frac{1}{\left(2 p_{2} A+p_{1}\right)}, i=0 .
\end{aligned}
$$




$$
\begin{aligned}
& =\frac{A}{\left(2 p_{2} A+p_{1}\right)}, i=1 . \\
& =\frac{A^{2}}{\left(2 p_{2} c+p_{1}\right)}, i=2 .
\end{aligned}
$$

The variance of $d_{5}$ depends on variances from all the factors in $d_{5}$.

$$
\begin{aligned}
& {\sigma_{d 5}}^{2}=\left(\frac{\partial d_{5}}{\partial d}\right)^{2} \sigma_{d}^{2}+\left(\frac{\partial d_{5}}{\partial b}\right)^{2} \sigma_{b}^{2}+\left(\frac{\partial d_{5}}{\partial C F_{C f}}\right)^{2}+\sigma_{C F_{C f}}{ }^{2} \\
& +\left(\frac{\partial d_{5}}{\partial C F_{\text {flow }}}\right)^{2} \sigma_{C F_{\text {flow }}}{ }^{2}+\left(\frac{\partial d_{5}}{\partial C F_{\text {flux }}}\right)^{2} \sigma_{C F_{f l u x}}{ }^{2} \\
& +\left(\frac{\partial d_{5}}{\partial C F_{\text {cycle }}}\right)^{2} \sigma_{C F_{C y c l e}}{ }^{2}+\left(\frac{\partial d_{5}}{\partial C F_{\text {norm }}}\right)^{2} \sigma_{C F_{n o r m}}^{2} \\
& \sigma_{d 5}{ }^{2}=\left(\frac{\partial d_{5}}{\partial d}\right)^{2} \sigma_{d}{ }^{2}+\left(\frac{\partial d_{5}}{\partial b}\right)^{2} \sigma_{b}{ }^{2}+\left(\frac{\partial d_{5}}{\partial C F_{C f}}\right)^{2} \sigma_{C F_{C f}}{ }^{2} \\
& +\left(\frac{\partial d_{5}}{\partial C F_{\text {flow }}}\right)^{2} \cdot\left[\left(\frac{\partial C F_{\text {flow }}}{\partial F}\right)^{2} \sigma_{F}^{2}+\sum_{i} \sum_{j}\left(\frac{\partial C F_{\text {flow }}}{\partial g_{i}}\right)\left(\frac{\partial C F_{\text {flow }}}{\partial g_{j}}\right) \sigma_{g_{i} g_{j}}\right] \\
& +\left(\frac{\partial d_{5}}{\partial C F_{f l u x}}\right)^{2} \cdot\left[\left(\frac{\partial C F_{f l u x}}{\partial m_{n}}\right)^{2} \sigma_{m_{n}}{ }^{2}+\sum_{i} \sum_{j}\left(\frac{\partial C F_{f l u x}}{\partial q_{i}}\right)\left(\frac{\partial C F_{f l u x}}{\partial q_{j}}\right) \sigma_{q_{i} q_{j}}\right] \\
& +\left(\frac{\partial d_{5}}{\partial C F_{\text {cycle }}}\right)^{2} \cdot\left[\left(\frac{\partial C F_{\text {cycle }}}{\partial D_{n}^{\prime}}\right)^{2} \sigma_{D_{n}^{\prime}}{ }^{2}+\left(\frac{\partial C F_{\text {cycle }}}{\partial D^{\prime}}\right)^{2} \sigma_{D^{\prime}}{ }^{2}\right] \\
& +\left(\frac{\partial d_{5}}{\partial C F_{\text {norm }}}\right)^{2} \cdot\left[\left(\frac{\partial C F_{n o r m}}{\partial D_{n}^{n}}\right)^{2} \sigma_{D_{n}^{*}}^{2}+\left(\frac{\partial C F_{\text {norm }}}{\partial D^{n}}\right)^{2} \sigma_{D^{*}}{ }^{2}\right]
\end{aligned}
$$


3. Random and Systematic Uncertainties. Equations (64), (66), and (71) are next placed into Eq. (63) and then split into the random and systematic variances.

$$
\begin{aligned}
& \sigma_{\mathrm{r}}^{2}=\left(\frac{\partial A}{\partial d_{5}}\right)^{2} \cdot\left\{\left(\frac{\partial d_{5}}{\partial d}\right)^{2} \cdot\left(\sigma_{d}^{2}+\sigma_{b}^{2}+\sigma_{C F_{C f}}{ }^{2}\right)+\frac{\partial d_{5}}{\partial C F_{f_{\text {fux }}}}\right. \text {. } \\
& \cdot\left[\left(\frac{\partial C F_{f l u x}}{\partial m_{n}}\right)^{2} \sigma_{m n}{ }^{2}+\sum_{i} \sum_{j}\left(\frac{\partial C F_{f l u x}}{\partial q_{i}}\right)\left(\frac{\partial C F_{f l u x}}{\partial q_{j}}\right) \sigma_{q_{i} q_{j}}\right] \\
& \left.+\left(\frac{\partial d_{5}}{\partial C F_{\text {cycle }}}\right)^{2} \cdot\left[\left(\frac{\partial C F_{\text {cycle }}}{\partial D^{\prime}{ }_{n}}\right)^{2} \sigma_{D^{\prime}{ }_{n}}{ }^{2}+\left(\frac{\partial C F_{\text {cycle }}}{\partial D^{\prime}}\right)^{2} \sigma_{D^{\prime}}{ }^{\prime}\right]\right\} . \\
& \sigma_{s}^{2}=\left(\frac{\partial A}{\partial d_{5}}\right)^{2}\left\{\left(\frac{\partial d_{5}}{\partial C F_{\text {flow }}}\right)^{2} \cdot\left[\left(\frac{\partial C F_{\text {flow }}}{\partial F}\right)^{2} \sigma_{F}^{2}+\sum_{i} \sum_{j}\left(\frac{\partial C F_{\text {flow }}}{\partial g_{i}}\right)\left(\frac{\partial C F_{\text {flow }}}{\partial g_{j}}\right) \sigma_{g_{i} g_{j}}\right]\right. \\
& \left.+\left(\frac{\partial d_{5}}{\partial C F_{n o r m}}\right)^{2} \cdot\left[\left(\frac{\partial C F_{n o r m}}{\partial D_{n}^{*}}\right)^{2} \sigma_{D_{n}^{N}}{ }^{2}+\left(\frac{\partial C F_{n o r m}}{\partial D^{n}}\right)^{2} \sigma_{D^{*}}^{2}\right]+\sum_{i} \sum_{j}\left(\frac{\partial d_{5}}{\partial p_{i}}\right)\left(\frac{\partial d_{5}}{\partial p_{j}}\right) \sigma_{p_{i} p_{j}}\right\} .
\end{aligned}
$$

The individual derivatives can be evaluated, both in general terms and for the expressions assumed earlier as examples.

a. Delayed-Neutron Count Rate.

$$
\begin{aligned}
& \left(\frac{\partial d_{5}}{\partial d}\right)=\frac{d_{5}}{(d-b)} . \\
& \sigma_{d}=\frac{\sqrt{D}}{T_{C}}=\sqrt{\frac{d}{T_{C}}} .
\end{aligned}
$$




\section{b. Background Subtraction.}

$$
\begin{aligned}
& \left(\frac{\partial d_{5}}{\partial b}\right)=\frac{-d_{5}}{(d-b)} . \\
& \sigma_{b}=\frac{\sqrt{B}}{T_{B}}=\sqrt{\frac{b}{T_{B}}} .
\end{aligned}
$$

c. Californium Decay.

$$
\begin{aligned}
& \left(\frac{\partial d_{5}}{\partial C F_{C f}}\right)=\frac{d_{5}}{C F_{C f}} . \\
& \sigma_{C f C F}{ }^{2}=\left(\lambda \cdot C F_{C f}\right)^{2} \sigma_{\lambda}{ }^{2}+\left(d d \cdot C F_{C f}\right)^{2} \sigma_{d d}{ }^{2} .
\end{aligned}
$$

d. Flow Rate.

$$
\begin{aligned}
& \left(\frac{\partial d_{5}}{\partial F}\right)=\left(\frac{\partial d_{5}}{\partial C F_{\text {flow }}}\right)\left(\frac{\partial C F_{\text {flow }}}{\partial F}\right)=\left(\frac{d_{5}}{C F_{\text {flow }}}\right)\left(2 g_{2} F+g_{1}\right) . \\
& \sigma_{F}=\text { instrument dependent } \\
& \left(\frac{\partial d_{5}}{\partial g_{i}}\right)=\left(\frac{\partial d_{5}}{\partial C F_{\text {flow }}}\right)\left(\frac{\partial C F_{\text {flow }}}{\partial g_{i}}\right)=\left(\frac{d_{5}}{C F_{\text {flow }}}\right)\left(\frac{\partial C F_{\text {flow }}}{\partial g_{i}}\right)=\left(\frac{d_{5}}{C F_{\text {flow }}}\right) \cdot X .
\end{aligned}
$$

where $X=1, F$, and $F^{2}$ for $i=0,1$, and 2 .

$$
\sigma_{g_{i} g_{j}}=\text { from curve-fitting process to find } g_{i}
$$


e. Flux Monitor.

$\left(\frac{\partial d_{5}}{\partial m_{1}}\right)=\left(\frac{\partial d_{5}}{\partial C F_{f l u x}}\right)\left(\frac{\partial C F_{f l u x}}{\partial m_{1}}\right)=\left(\frac{d_{5}}{C F_{f l u x}}\right)\left(\frac{\partial C F_{f l u x}}{\partial m_{1}}\right)=\left(\frac{d_{5}}{C F_{f l u x}}\right)\left(\frac{-q}{m_{1}}\right)$.

$\sigma_{m_{1}}=\sqrt{\frac{m_{1}}{T_{1}}}$

$\left(\frac{\partial d_{5}}{\partial m_{n}}\right)=\left(\frac{\partial d_{5}}{\partial C F_{f l u x}}\right)\left(\frac{\partial C F_{f l u x}}{\partial m_{n}}\right)=\left(\frac{d_{5}}{C F_{f l u x}}\right)\left(\frac{\partial C F_{f l u x}}{\partial m_{n}}\right)=\left(\frac{d_{5}}{C F_{f l u x}}\right)\left(\frac{q}{m_{n}}\right)$.

$\sigma_{m_{n}}=\sqrt{\frac{m_{n}}{T_{l_{n}}}}$

$\left(\frac{\partial d_{5}}{\partial q_{i}}\right)=\left(\frac{\partial d_{5}}{\partial C F_{f l u x}}\right)\left(\frac{\partial C F_{f l u x}}{\partial q_{i}}\right)=\left(\frac{d_{5}}{C F_{f l u x}}\right)\left(\frac{\partial C F_{f l u x}}{\partial q_{i}}\right)=d_{5} \ln \left(\frac{m_{n}}{m_{1}}\right)$.

$\sigma_{q}=$ application dependent if $q \neq 1 ; 0$ if $q=1$.

$T_{I_{n}}$ in $\sigma_{m_{n}}$ is the total nominal irradiation (and count) time.

\section{f. Cycle Correction.}

$\left(\frac{\partial d_{5}}{\partial D_{n}^{\prime}}\right)=\left(\frac{\partial d_{5}}{\partial C F_{\text {cycle }}}\right)\left(\frac{\partial C F_{\text {cycle }}}{\partial D_{n}^{\prime}}\right)=\left(\frac{d_{5}}{C F_{c y c l e}}\right)\left(\frac{\partial C F_{\text {cycle }}}{\partial D_{n}^{\prime}}\right)=\frac{d_{5}}{\left(C F_{\text {cycle }} \cdot D_{n}^{\prime}\right)}$.

$\sigma_{D_{n}}=0$

$\left(\frac{\partial d_{5}}{\partial D^{\prime}}\right)=\left(\frac{\partial d_{5}}{\partial C F_{\text {cycle }}}\right)\left(\frac{\partial C F_{\text {cycle }}}{\partial D^{\prime}}\right)=\left(\frac{d_{5}}{C F_{\text {cycle }}}\right)\left(\frac{\partial C F_{\text {cycle }}}{\partial D^{\prime}}\right)=\frac{-d_{5}}{D^{\prime}}$. 


$$
\sigma_{D^{\prime}} \cong 0
$$

The uncertainties of $D_{n}^{\prime}$ and $D^{\prime}$ are both essentially zero. $D_{n}^{\prime}$ is a calculated item and $D^{\prime}$ is a function of times and nuclear parameters that are accurately known.

g Normalization.

$$
\begin{aligned}
& \left(\frac{\partial d_{5}}{\partial D_{n}^{N}}\right)=\left(\frac{\partial d_{5}}{\partial C F_{n o r m}}\right)\left(\frac{\partial C F_{n o r m}}{\partial D_{n}^{N}}\right)=\left(\frac{d_{5}}{C F_{n o r m}}\right)\left(\frac{\partial C F_{n o r m}}{\partial D_{n}^{N}}\right)=\frac{d_{5}}{D_{n}^{\prime \prime}} \\
& \sigma_{D_{n}^{N}}=\text { method dependent. } \\
& \left(\frac{\partial d_{5}}{\partial D^{\prime \prime}}\right)=\left(\frac{\partial d_{5}}{\partial C F_{n o r m}}\right)\left(\frac{\partial C F_{n o r m}}{\partial D^{N}}\right)=\left(\frac{d_{5}}{C F_{n o r m}}\right)\left(\frac{\partial C F_{n o r m}}{\partial D^{\prime \prime}}\right)=\frac{-d_{5}}{D^{\prime \prime}} \\
& \sigma_{D^{*}}=\text { method dependent. }
\end{aligned}
$$

If the normalization is based on routine counting of a standard, conventional counting statistics can be applied for the uncertainties of $D_{n}^{\prime \prime}$ and $D^{\prime \prime}$.

h. Calibration.

$$
\begin{aligned}
\left(\frac{\partial d_{5}}{\partial p_{i}}\right)=\left(\frac{\partial f_{\text {calib }}}{\partial p_{i}}\right) & =1, i=0 \\
& =A, i=1 \\
& =A^{2}, i=2 .
\end{aligned}
$$

$$
\sigma_{p_{i}}{ }^{2}=\text { from curve fitting procedure }
$$


The random variance of Eq. (70) with the above derivatives can be made more explicit.

$$
\begin{aligned}
&\left(\frac{\partial f_{\text {calib }}}{\partial A}\right) \sigma_{r}^{2}=\left[\frac{d_{5}}{(d-b)}\right]^{2} \cdot\left(\frac{d}{T_{C}}+\frac{b}{T_{B}}\right) \\
&+\left(\frac{d_{5}}{C F_{C f}}\right)^{2} \cdot\left[\left(\lambda \cdot C F_{C f}\right)^{2} \sigma_{\lambda}^{2}+\left(d d \cdot C F_{C f}\right)^{2} \sigma_{d d^{2}}^{2}\right] \\
&+\left(\frac{d_{5}}{C F_{f l u x}}\right)^{2} \cdot\left[\left(\frac{\partial C F_{f l u x}}{\partial_{m_{n}}}\right)^{2}+\left(\frac{\partial C F_{f l u x}}{\partial_{m_{1}}}\right)^{2}+\sum_{i} \sum_{j}\left(\frac{\partial C F_{f l u x}}{q_{i}}\right)\left(\frac{\partial C F_{f l u x}}{q_{j}}\right) \sigma_{q_{i} q_{j}}\right] \\
&+\left(\frac{d_{5}}{C F_{c y c l e}}\right)^{2} \cdot\left[\left(\frac{\partial C F_{c y c l e}}{\partial D_{n}^{\prime}}\right)^{2}+\left(\frac{\partial C F_{c y c l e}}{\partial D_{n}^{\prime}}\right)^{2}\right] .
\end{aligned}
$$

With the specialized expressions given earlier, and the uncertainties in $\lambda$ and $d d$ taken as zero, this becomes,

$$
\begin{aligned}
&\left(2 p_{2} A+p_{1}\right) \sigma_{r}^{2}=\left(\frac{d_{5}}{d_{1}}\right)^{2} \cdot\left(C F_{C f} \cdot \frac{d}{T_{C}}+\frac{b}{T_{B}}\right) \\
&+d_{5}^{2} \cdot\left\{\left(\frac{q^{2}}{m_{n} T_{I_{n}}}\right)+\left(\frac{q^{2}}{m_{1} T_{I}}\right)+\left[\ln \left(\frac{m_{n}}{m_{1}}\right)\right]^{2} \sigma_{q}^{2}\right\} \\
&+d_{5}^{2} \cdot\left[\left(\frac{\sigma_{D_{n}^{\prime}}^{\prime}}{D_{n}^{\prime}}\right)^{2}+\left(\frac{\sigma_{D} \cdot}{D^{\prime}}\right)^{2}\right]
\end{aligned}
$$

The systematic variance of Eq. (71) can also be expanded. The uncertainty in the cycle correction factor will be taken as zero in general. 


$$
\begin{aligned}
\left(\frac{\partial f_{\text {calib }}}{\partial A}\right) \sigma_{s}^{2}= & \left(\frac{d_{5}}{C F_{\text {flow }}}\right)^{2}\left[\left(\frac{\partial C F_{f l o w}}{\partial F}\right)^{2} \sigma_{F}^{2}\right] \\
& +\sum_{i} \sum_{j}\left(\frac{\partial C F_{f l o w}}{\partial g_{i}}\right)\left(\frac{\partial C F_{f l o w}}{\partial g_{j}}\right) \sigma_{g_{i} g_{j}} \cdot \\
& +\left(\frac{d_{5}}{C F_{n o r m}}\right)^{2}\left[\left(\frac{\partial C F_{n o r m}}{\partial D_{n}^{\prime \prime}}\right)^{2} \sigma_{D_{n}^{\prime \prime}}{ }^{2}+\left(\frac{\partial C F_{n o r m}}{\partial D^{\prime \prime}}\right)^{2} \sigma_{D^{\prime \prime}}{ }^{2}\right] \\
& +\sum_{i} \sum_{j}\left(\frac{\partial f_{\text {calib }}}{\partial p_{i}}\right)\left(\frac{\partial f_{c a l i b}}{\partial p_{j}}\right) \sigma_{p_{i} p_{j}} .
\end{aligned}
$$

Using the specialized expressions for the corrections, the systematic variance becomes

$$
\begin{aligned}
\left(2 p_{2} \mathrm{~A}+p_{1}\right) \sigma_{s}{ }^{2} & =\left(\frac{d_{5}}{C F_{\text {flow }}}\right)^{2} \\
& \cdot\left[\left(2 g_{2} \mathrm{~F}+g_{1}\right)^{2} \sigma_{F}^{2}+\sigma_{g_{0}}{ }^{2}+F^{2}{\sigma_{g_{1}}}^{2}+F^{4}{\sigma_{g_{2}}}^{2}+F \sigma_{g_{0} g_{1}}+F^{2} \sigma_{g_{0} g_{2}}+F^{3} \sigma_{g_{1} g_{2}}\right] \\
& +\left(\frac{d_{5}}{C F_{n o r m}}\right)^{2} \cdot\left[\left(\frac{1}{D^{\prime \prime}}\right)^{2} \sigma_{D_{n}^{*}}^{2}+\left(\frac{D_{n}^{\prime \prime}}{D^{\prime \prime}}\right) \sigma_{D_{n}^{*}}{ }^{2}\right] \\
& +\sigma_{p_{0}}^{2}+A^{2} \sigma_{p_{1}}^{2}+A^{4} \sigma_{p_{2}}^{2}+A \sigma_{p_{0} p_{1}}+A^{2} \sigma_{p_{0} p_{2}}+A^{3} \sigma_{p_{1} p_{2}}
\end{aligned}
$$

In practice, normalization is not usually necessary and the material is usually stationary, so the only contributor to the systematic uncertainty is the calibration curve.

The random and systematic variances are added as in Eq. (60) to give the total variance of the assay result. 
4. Total Uncertainty with Bias Correction. If a bias correction is used, as indicated in Eq. (59), the total variance is increased.

$$
\sigma_{A_{b c}}^{2}=\left(\frac{\partial A_{b c}}{\partial A}\right)^{2} \sigma_{A}^{2}+\sum_{i} \sum_{j}\left(\frac{\partial A_{b c}}{\partial b_{i}}\right)\left(\frac{\partial A_{b c}}{\partial b_{j}}\right) \sigma_{b_{i} b_{j}}
$$

\section{Generalized Numerical Techniques}

Some of the procedures needed can be done in generalized ways to avoid writing specialized codes.

1. Interpolation from the Calibration Curve. The basis of this procedure is the Newton-Raphson iterative technique for finding the root of a function. Let $d$ be the measured detector response and $A$ the assay value to be found from the calibration curve $d=f(A,\{p\})$, where the $\{p\}$ are parameters in the function. The root $A$ of $g(A)=d-f(A,\{p\})=0$ is to be found. Assume some initial estimate $A_{1}$ for $A$; it need not be very accurate. Calculate successive values of $A$ from

$$
A_{i+1}=A_{i}-\frac{\left[d-f\left(A_{i}\right)\right]}{f\left(A_{i}\right)}
$$

Here $f^{\prime}$ is the derivative of $f$ with respect to $A$ and can be found numerically (Sec. VIII.C.3). When $\left|\left(A_{i+1}-A_{i}\right) / A_{i+1}\right|<0.0001$, the iterations have converged to the answer with sufficient accuracy.

2. Uncertainty in an Interpolated Value. From a corrected response $d \pm \sigma$, the corresponding interpolated concentration $A$ is interpolated as described in Sec. VIII.C.2.

The uncertainty in $A$ is calculated by propagation of errors, taking the derivatives numerically (Sec. VIII.C.3); changing the calibration function will not require any changes to the code for calculating the uncertainty:

$$
\sigma_{A}=\left(\frac{\partial A}{\partial d}\right)^{2} \sigma_{d}^{2}+\sum_{i} \sum_{j}\left(\frac{\partial A}{\partial p_{i}}\right)\left(\frac{\partial A}{\partial p_{j}}\right) \sigma_{p_{i} p_{j}}
$$


From the relation $d=f(A,\{p\})$, the derivatives are

$$
\left(\frac{\partial A}{\partial d}\right)=\frac{1}{\left(\frac{\partial d}{\partial A}\right)}
$$

and

$$
\left(\frac{\partial A}{\partial d}\right)=\frac{\left(\frac{\partial d}{\partial p}\right)}{\left(\frac{\partial d}{\partial A}\right)} .
$$

3. Numerical Derivatives. Derivatives of more than one function are needed in several places in the analysis. To avoid having to develop analytical expressions for the many different derivatives and then insert them into the code, a generalized approach can be taken. The functions are given in simple subroutines and their derivatives are taken numerically when needed.

The numerical derivative of a function $f(x)$ can be approximated by

$$
f^{\prime}(x)=\frac{f\left(x+\frac{\Delta x}{2}\right)-f\left(x-\frac{\Delta x}{2}\right)}{\Delta x}
$$

A value of $\Delta x=x / 1000$ has been found practical; if $x$ is zero, then $\Delta x=0.001$ can be used. Smaller values of $\Delta x$ may actually decrease the precision because of round-off losses within the computer.

\section{ACKNOWLEDGMENT}

The work described in this report is a summary and continuation of the development of shuffler technology spearheaded for many years at Los Alamos by Howard Menlove, George Eccleston, Tom Crane, and Norbert Ensslin.

Critical readings of this manuscript by Norbert Ensslin and Howard Menlove were most helpful. 


\section{APPENDIX}

\section{DELAYED-NEUTRON DATA}

Neutron energies are labeled in this appendix as thermal $(0.025 \mathrm{eV})$, fast (fission spectrum), and reactor (the neutron spectrum in a light-water reactor).

TABLE A-1. Thorium-232

Values in [ ] are deduced from other values in this table.

\begin{tabular}{|c|c|c|c|c|c|c|c|c|}
\hline$E_{n}$ & Group & & $\lambda_{j}$ & $\begin{array}{c}T_{1 / 2} \\
(\mathrm{~s})\end{array}$ & $\beta_{j}$ & $\beta_{j} / \Sigma \beta_{j}$ & & $\beta_{j} v$ \\
\hline \multirow{8}{*}{$\begin{array}{l}\text { Fast }^{5} \\
3.5 \mathrm{McV} \\
v=2.44 \pm 0.15\end{array}$} & 1 & 0.0124 & $4 \pm 0.0002$ & $56.03 \pm 0.95$ & {$[0.000693]$} & $0.034 \pm 0.002$ & 0.00169 & \pm 0.00012 \\
\hline & 2 & 0.0334 & $4 \pm 0.0011$ & $20.75 \pm 0.66$ & {$[0.00305]$} & $0.150 \pm 0.005$ & 0.00744 & \pm 0.00037 \\
\hline & 3 & 0.121 & \pm 0.005 & $5.74 \pm 0.24$ & {$[0.00315]$} & $0.155 \pm 0.021$ & 0.00769 & \pm 0.00108 \\
\hline & 4 & 0.321 & \pm 0.011 & $2.16 \pm 0.08$ & {$[0.00907]$} & $0.446 \pm 0.015$ & 0.02212 & \pm 0.00110 \\
\hline & 5 & 1.21 & \pm 0.090 & $0.571 \pm 0.042$ & {$[0.00350]$} & $0.172 \pm 0.013$ & 0.00853 & $\therefore 0.00073$ \\
\hline & 6 & 3.29 & \pm 0.297 & $0.211 \pm 0.019$ & {$[0.000873]$} & $0.043 \pm 0.006$ & 0.00213 & \pm 0.00031 \\
\hline & Alla & & & & $0.0203 \pm 0.0015$ & 1.000 & 0.0496 & \pm 0.0020 \\
\hline & Alla & & & & & & 0.0497 & \pm 0.0027 \\
\hline \multirow[t]{8}{*}{ Fast ${ }^{6}$} & 1 & \multicolumn{2}{|c|}{$0.0124 \pm 0.002$} & \multicolumn{2}{|l|}{$[55.90]$} & {$[0.00351]$} & \multicolumn{2}{|c|}{$0.000190 \pm 0.00013$} \\
\hline & 2 & \multicolumn{2}{|c|}{$0.0334 \pm 0.0011$} & \multicolumn{2}{|l|}{$[20.75]$} & {$[0.155]$} & \multicolumn{2}{|c|}{$0.00836 \pm 0.00042$} \\
\hline & 3 & 0.121 & \pm 0.005 & \multicolumn{2}{|l|}{ [ 5.73 ] } & {$[0.1601$} & \multicolumn{2}{|c|}{$0.00864 \pm 0.00121$} \\
\hline & 4 & 0.321 & \pm 0.011 & \multicolumn{2}{|l|}{$\mid 2.16$ i } & {$[0.460]$} & \multicolumn{2}{|c|}{$0.0249 \pm 0.0012$} \\
\hline & 5 & 1.21 & \pm 0.090 & \multicolumn{2}{|l|}{$|0.573|$} & {$[0.177]$} & \multicolumn{2}{|c|}{$0.00958 \pm 0.00082$} \\
\hline & 6 & 3.29 & \pm 0.30 & \multirow{2}{*}{\multicolumn{2}{|c|}{$[0.211]$}} & $|0.0442|$ & 0.00239 & \pm 0.00035 \\
\hline & All & & & & & $11.000 j$ & \multicolumn{2}{|c|}{$0.0527 \pm 0.0040$} \\
\hline & All & & & & & & \multicolumn{2}{|c|}{$[0.0541]$} \\
\hline Fast 24 & All & & & & & & 0.0547 & \pm 0.0012 \\
\hline Reactor ${ }^{24}$ & All & & & & & & 0.0545 & \pm 0.0011 \\
\hline
\end{tabular}

a Average of several laboratories' values.

TABLE A-1I. Uranium-232

Values in [ ] are deduced from other values in this table.

\begin{tabular}{|c|c|c|c|c|c|c|}
\hline$E_{n}$ & Group & $\begin{array}{c}\lambda_{j} \\
\left(\mathrm{~s}^{-1}\right)\end{array}$ & $\begin{array}{c}T_{1 / 2} \\
(\mathrm{~s}) \\
\end{array}$ & A & $\beta_{j} \Sigma \beta_{j}$ & $\beta_{j} v$ \\
\hline \multirow[t]{6}{*}{ Thermal ${ }^{6}$} & 1 & $0.01276 \pm 0.00004$ & $\{54.32\}$ & & {$[0.120]$} & $0.0010524 \pm 0.000040$ \\
\hline & 2 & $0.03502 \pm 0.00029$ & {$[19.79\}$} & & [0.299] & $0.00131 \pm 0.00010$ \\
\hline & 3 & $0.1439 \pm 0.005$ & | 4.817| & & {$[0.307]$} & $0.00134 \pm 0.00014$ \\
\hline & 4 & $0.396 \pm 0.045$ & [ $1.75 \mathrm{j}$ & & {$[0.258]$} & $0.000113 \pm 0.00012$ \\
\hline & 5 & $1.35^{\mathrm{a}}$ & {$[0.513]$} & & {$[0.016]$} & $0.000015 \pm 0.00039$ \\
\hline & 6 & - & $1-1$ & & $1 \ldots 1$ & $\cdots$ \\
\hline All & & & & & 11.00001 & $0.00 .437 \pm 0.000 .33$ \\
\hline
\end{tabular}

Assumed values. 
TABLE A-11I. Uranium-233

Values in 1 I are deduced from other values in this table.

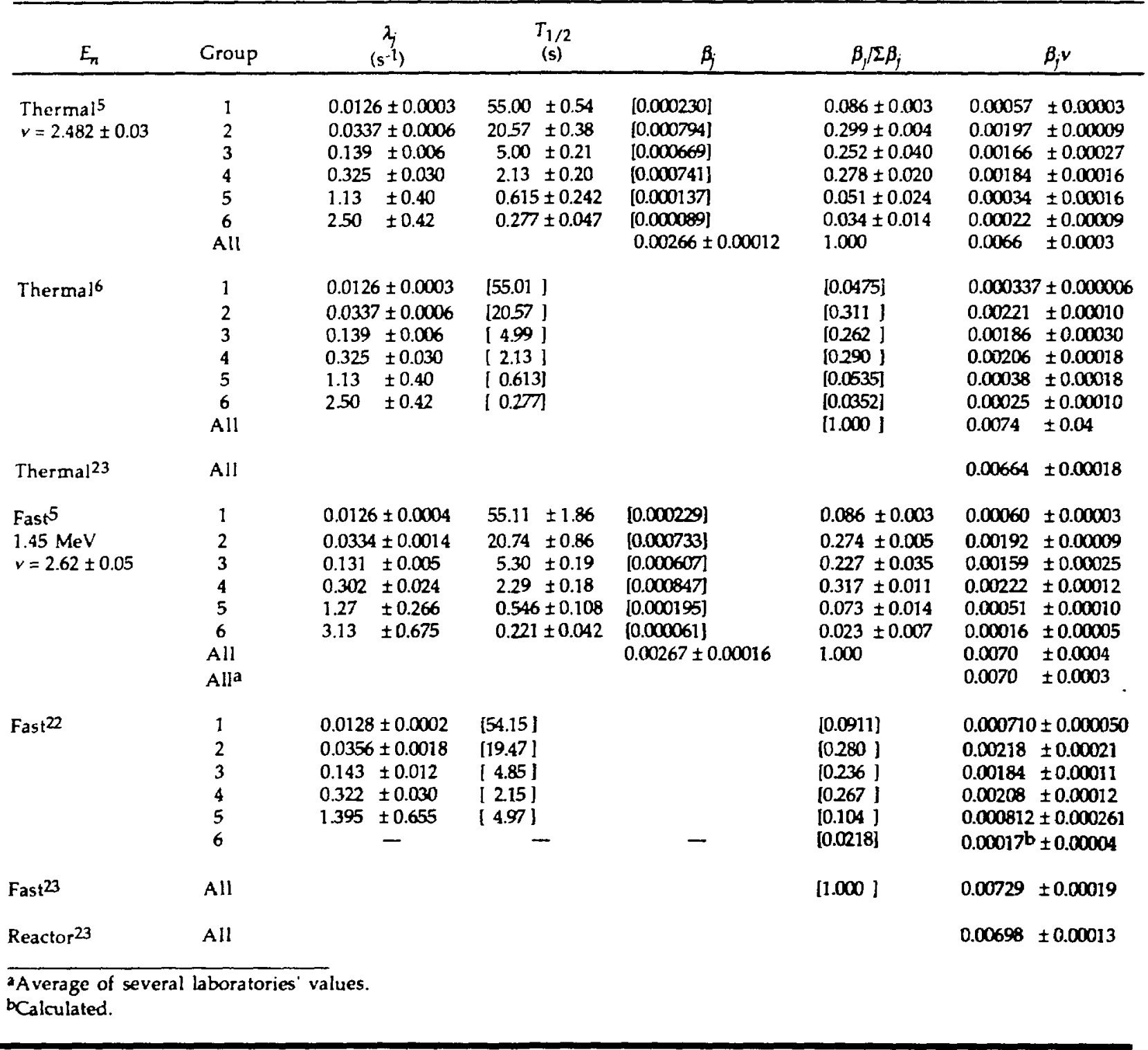


TABLE A-IV. Uranium-234

Values in [ ] are deduced from other values in this table.

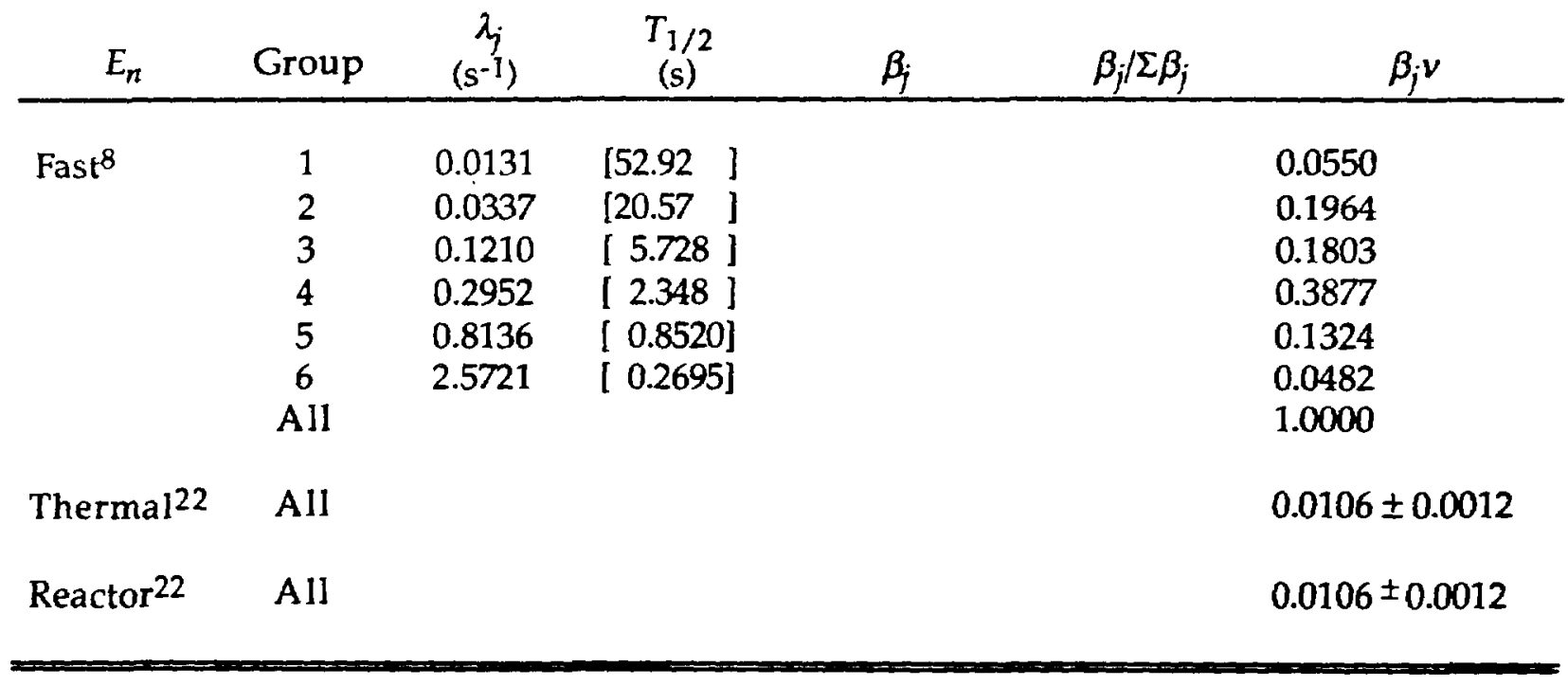


TABLE A-V. Uranium-235

Values in [ ] are deduced from other values in this table.

$v=(2.432 \pm 0.008)+(0.066 \pm 0.016) E_{n}, 0<E_{n}<1 \mathrm{MeV}(\operatorname{Ref}$. 4)

$v=(2.349 \pm 0.012)+(0.150 \pm 0.003) E_{n}, E_{n}>1 \mathrm{MeV}$ (Ref. 4)

\begin{tabular}{|c|c|c|c|c|c|c|c|}
\hline$E_{n}$ & Group & & $\begin{array}{r}\lambda \\
\left(s^{-1}\right) \\
\end{array}$ & $\begin{array}{l}T_{1 / 2} \\
(\mathrm{~s})\end{array}$ & $\beta_{j}$ & $\beta_{j} / \Sigma \beta_{j}$ & $\beta_{j} v$ \\
\hline $\begin{array}{l}\text { Thermal } 5 \\
v=2.43 \pm 0.02\end{array}$ & $\begin{array}{c}1 \\
2 \\
3 \\
4 \\
5 \\
6 \\
\text { All }\end{array}$ & $\begin{array}{l}0.01244 \\
0.03051 \\
0.1114 \\
0.3014 \\
1.136 \\
3.014\end{array}$ & $\begin{array}{l}4 \pm 0.0003 \\
1 \pm 0.0010 \\
\pm 0.004 \\
\pm 0.011 \\
\pm 0.15 \\
\pm 0.29\end{array}$ & $\begin{aligned} & 55.72 \pm 1.28 \\
& 22.72 \pm 0.71 \\
& 6.22 \pm 0.23 \\
& 2.30 \pm 0.09 \\
& 0.610 \pm 0.083 \\
& 0.230 \pm 0.025\end{aligned}$ & $\begin{array}{l}{[0.00021]} \\
{[0.00142]} \\
{[0.00128]} \\
{[0.00257]} \\
{[0.00075]} \\
{[0.00027]} \\
0.00650 \pm 0.00021\end{array}$ & $\begin{array}{l}0.033 \pm 0.003 \\
0.219 \pm 0.009 \\
0.19: \pm 0.022 \\
0.395 \pm 0.011 \\
0.115 \pm 0.009 \\
0.042 \pm 0.008 \\
1.000\end{array}$ & $\begin{array}{l}0.00052 \pm 0.00005 \\
0.00346 \pm 0.00018 \\
0.00310 \pm 0.00036 \\
0.00624 \pm 0.00026 \\
0.00182 \pm 0.00015 \\
0.00066 \pm 0.00008 \\
0.0158 \pm 0.0005\end{array}$ \\
\hline Thermal 6 & $\begin{array}{c}1 \\
2 \\
3 \\
4 \\
5 \\
6 \\
\text { All }\end{array}$ & $\begin{array}{l}0.0127 \\
0.0317 \\
0.115 \\
0.311 \\
1.40 \\
3.87\end{array}$ & $\begin{array}{l} \pm 0.0003 \\
\pm 0.038 \\
\pm 0.004 \\
\pm 0.012 \\
\pm 0.12 \\
\pm 0.55\end{array}$ & 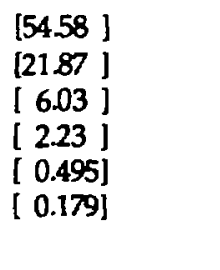 & $\begin{array}{l}{[0.00023]} \\
{[0.00152]} \\
{[0.00136]} \\
{[0.00274]} \\
{[0.00080]} \\
{[0.00029]} \\
{[0.00694]}\end{array}$ & $\begin{array}{l}{[0.0329]} \\
{[0.219]} \\
{[0.196]} \\
{[0.395]} \\
{[0.115]} \\
{[0.0419]} \\
{[1.000]}\end{array}$ & $\begin{array}{l}0.00055 \pm 0.00005 \\
0.00366 \pm 0.00038 \\
0.00328 \pm 0.00038 \\
0.00660 \pm 0.00027 \\
0.00192 \pm 0.00016 \\
0.00070 \pm 0.00008 \\
0.0167 \pm 0.0007\end{array}$ \\
\hline Thermal23 & All & & & & [0.00686] & & $0.01654 \pm 0.00042$ \\
\hline $\begin{array}{l}\text { Fast }^{5} \\
1.45 \mathrm{MeV} \\
v=2.57 \pm 0.04\end{array}$ & $\begin{array}{c}1 \\
2 \\
3 \\
4 \\
5 \\
6 \\
\text { All } \\
\text { Alla } \\
\text { All }\end{array}$ & $\begin{array}{l}0.0127 \\
0.0317 \\
0.115 \\
0.311= \\
1.40= \\
3.87\end{array}$ & $\begin{array}{l} \pm 0.0002 \\
\pm 0.0008 \\
\pm 0.003 \\
\pm 0.008 \\
\pm 0.081 \\
\pm 0.369\end{array}$ & $\begin{aligned} & 54.51 \pm 0.94 \\
& 21.84 \pm 0.54 \\
& 6.00 \pm 0.17 \\
& 2.23 \pm 0.06 \\
& 0.496 \pm 0.029 \\
& 0.179 \pm 0.017\end{aligned}$ & $\begin{array}{l}{[0.000245]} \\
{[0.00137]} \\
{[0.00121]} \\
{[0.00261]} \\
{[0.000821]} \\
{[0.000167]} \\
0.00641 \pm 0.00022\end{array}$ & $\begin{array}{l}0.038 \pm 0.003 \\
0.213 \pm 0.005 \\
0.188 \pm 0.016 \\
0.407 \pm 0.007 \\
0.128 \pm 0.008 \\
0.026 \pm 0.003 \\
1.000\end{array}$ & $\begin{array}{l}0.00063 \pm 0.00005 \\
0.00351 \pm 0.00011 \\
0.00310 \pm 0.00028 \\
0.00672 \pm 0.00023 \\
0.00211 \pm 0.00015 \\
0.00043 \pm 0.00005 \\
0.0165 \pm 0.0005 \\
0.0164 \pm 0.0005 \\
0.01714 \pm 0.00022\end{array}$ \\
\hline Reactor 23 & All & & & & & & $0.01697 \pm 0.00020$ \\
\hline
\end{tabular}

Average of several laboratories' values. 
TABLE A-VI. Uranium-236

Values in [ ] are deduced from other values in this table.

\begin{tabular}{|c|c|c|c|c|c|c|}
\hline$E_{n}$ & Group & $\begin{array}{c}\lambda_{j} \\
\left(\mathbf{s}^{-1}\right)\end{array}$ & $\begin{array}{c}T_{1 / 2} \\
(\mathrm{~s})\end{array}$ & $\beta_{j}$ & $\beta_{j} / \Sigma \beta_{j}$ & $\beta_{j} v$ \\
\hline \multirow[t]{7}{*}{ Fast $^{8}$} & 1 & 0.0134 & {$[51.73]$} & & 0.0302 & \\
\hline & 2 & 0.0322 & {$[21.53]$} & & 0.1722 & \\
\hline & 3 & 0.1202 & {$\left[\begin{array}{l}5.767 \\
]\end{array}\right.$} & & 0.1619 & \\
\hline & 4 & 0.3113 & {$[2.227]$} & & 0.3841 & \\
\hline & 5 & 0.8794 & {$[0.7882]$} & & 0.1775 & \\
\hline & 6 & 2.8405 & {$[0.24402]$} & & 0.0741 & \\
\hline & All & & & & 1.000 & \\
\hline Fast 23 & All & & & & & $0.0231 \pm 0.0026$ \\
\hline Reactor 23 & All & & & & & $0.0231 \pm 0.0026$ \\
\hline
\end{tabular}

TABLE A-VII. Uranium-238

Values in [ ] are deduced from other values in this table.

\begin{tabular}{|c|c|c|c|c|c|c|c|c|}
\hline$E_{n}$ & Group & & $\begin{array}{c}\lambda_{j} \\
\left(\mathrm{~s}^{-1}\right) \\
\end{array}$ & $\begin{array}{c}T_{1 / 2} \\
(\mathrm{~s}) \\
\end{array}$ & $\beta_{j}$ & $\beta_{j} / \Sigma \beta_{j}$ & & $\beta_{j} v$ \\
\hline Fast $^{5}$ & 1 & 0.0132 & \pm 0.0003 & $52.38 \pm 1.29$ & {$[0.00019]$} & $0.013 \pm 0.001$ & 0.00054 & \pm 0.00005 \\
\hline $3.01 \mathrm{MeV}$ & 2 & 0.0321 & \pm 0.0006 & $21.58 \pm 0.39$ & {$[0.00202\}$} & $0.137 \pm 0.002$ & 0.00564 & \pm 0.00025 \\
\hline \multirow[t]{6}{*}{$v=2.79 \pm 0.10$} & 3 & 0.139 & \pm 0.005 & $5.00 \pm 0.19$ & [0.00239] & $0.162 \pm 0.020$ & 0.00667 & \pm 0.00087 \\
\hline & 4 & 0.358 & \pm 0.014 & $1.93 \pm 0.07$ & {$[0.00573]$} & $0.388 \pm 0.012$ & 0.01599 & \pm 0.00081 \\
\hline & 5 & 1.41 & \pm 0.067 & $0.490 \pm 0.023$ & {$[0.00332]$} & $0.225 \pm 0.013$ & 0.00927 & \pm 0.00060 \\
\hline & 6 & 4.02 & \pm 0.214 & $0.172 \pm 0.009$ & {$[0.00111]$} & $0.075 \pm 0.005$ & 0.00309 & \pm 0.00024 \\
\hline & Alla & & & & $0.0148 \pm 0.0008$ & 1.000 & 0.0412 & \pm 0.0017 \\
\hline & All^ & & & & & & 0.0398 & \pm 0.0013 \\
\hline \multirow[t]{7}{*}{ Fast ${ }^{6}$} & 1 & 0.0132 & \pm 0.0003 & [52.51] & & [0.0130] & 0.000577 & $7 \pm 0.00004$ \\
\hline & 2 & 0.0321 & \pm 0.006 & $\{21.59\}$ & & {$[0.137]$} & 0.00608 & \pm 0.00009 \\
\hline & 3 & 0.139 & \pm 0.005 & [ 4.99 ] & & {$[0.162]$} & 0.00719 & \pm 0.00089 \\
\hline & 4 & 0.358 & \pm 0.014 & [ 1.94 ] & & [0.388 ] & 0.0172 & \pm 0.0005 \\
\hline & 5 & 1.41 & \pm 0.07 & {$[0.492]$} & & {$[0.225]$} & 0.0100 & \pm 0.0006 \\
\hline & 6 & 4.02 & \pm 0.21 & {$[0.172\}$} & & {$[0.0744]$} & 0.0033 & \pm 0.0002 \\
\hline & All & & & & & {$[1.000]$} & 0.0444 & \pm 0.0023 \\
\hline Fast $^{23}$ & All & & & & & & 0.04510 & \pm 0.00061 \\
\hline Combined 23 & All & & & & & & 0.04508 & \pm 0.00060 \\
\hline
\end{tabular}

'A verage of several laboratories' values. 
TABLE A-VIII. Neptunium-237

Values in [ ] are deduced from other values in this table.

\begin{tabular}{|c|c|c|c|c|c|c|c|c|}
\hline$E_{n}$ & Group & & $\begin{array}{c}\lambda_{j} \\
\left(\mathbf{s}^{-i}\right)\end{array}$ & $\begin{array}{c}T_{1 / 2} \\
(s)\end{array}$ & $\beta$ & $\beta_{j} / \Sigma \beta_{j}$ & & $\beta_{j} v$ \\
\hline \multirow[t]{7}{*}{ Thermal $^{6}$} & 1 & 0.01258 & $8 \pm 0.00004$ & [55.10 ] & {$[0.000136]$} & {$[0.0348]$} & 0.0003683 & $3 \pm 0.000034$ \\
\hline & 2 & 0.0306 & \pm 0.00034 & [22.65 ] & {$[0.000904]$} & {$[0.230]$} & 0.00244 & \pm 0.00024 \\
\hline & 3 & 0.0653 & \pm 0.016 & {$[10.6 \mathrm{j}$} & {$[0.000259]$} & {$[0.0660]$} & 0.00070 & \pm 0.00033 \\
\hline & 4 & 0.139 & \pm 0.019 & {$[4.99$ ] } & {$[0.000567]$} & {$[0.144]$} & 0.00153 & \pm 0.00065 \\
\hline & 5 & 0.328 & \pm 0.030 & {$[2.11]$} & {$[0.00157]$} & {$[0.400]$} & 0.00424 & \pm 0.00053 \\
\hline & 6 & 1.62 & \pm 0.69 & {$[0.428]$} & {$[0.000489]$} & {$[0.125]$} & 0.00132 & \pm 0.00031 \\
\hline & All & & & & {$[0.0003925]$} & {$[1.000]$} & 0.01068 & \pm 0.00098 \\
\hline \multirow[t]{7}{*}{ Fast 22} & 1 & 0.0127 & \pm 0.0002 & [54.58] & & {$[0.0402]$} & 0.000490 & \pm 0.000031 \\
\hline & 2 & 0.0318 & \pm 0.0006 & {$[21.80]$} & & {$[0.254]$} & 0.00310 & \pm 0.00009 \\
\hline & 3 & 0.123 & \pm 0.002 & {$[5.64]$} & & {$[0.206]$} & 0.00251 & \pm 0.00009 \\
\hline & 4 & 0.318 & \pm 0.004 & [ 2.18 ] & & {$[0.372]$} & 0.00454 & \pm 0.00014 \\
\hline & 5 & 1.03 & \pm 0.17 & {$[0.673]$} & & {$[0.106]$} & 0.00129 & \pm 0.00012 \\
\hline & 6 & & - & - & & {$[0.0221]$} & $0.00027 a$ & \pm 0.00007 \\
\hline & All & & & & & {$[1.000]$} & 0.0122 & \pm 0.0003 \\
\hline
\end{tabular}

acalculated. 
TABLE A-IX. Plutonium-238

Values in [ ] are deduced from other values in this table.

\begin{tabular}{|c|c|c|c|c|c|c|c|c|}
\hline \multirow{4}{*}{\begin{tabular}{l}
\multicolumn{1}{c}{$E_{n}$} \\
Fast $^{6}$ \\
$2 \mathrm{MeV}$ \\
$v=2.62^{5}$
\end{tabular}} & \multirow{2}{*}{$\frac{\text { Group }}{1}$} & \multicolumn{2}{|c|}{$\begin{array}{c}\lambda_{j} \\
\left\langle\mathbf{s}^{-1}\right)\end{array}$} & $\begin{array}{c}T_{1 / 2} \\
(\mathrm{~s})\end{array}$ & $\beta_{j}$ & $\beta_{j} / \Sigma \beta_{j}$ & \multicolumn{2}{|r|}{$\beta_{j} v$} \\
\hline & & 0.01262 & \pm 0.00013 & [54.92] & {$[0.000075]$} & {$[0.042]$} & 0.000197 & \pm 0.000031 \\
\hline & 2 & 0.03026 & \pm 0.00035 & {$[22.91]$} & [0.000542] & [0.308 ] & 0.00142 & \pm 0.00022 \\
\hline & 3 & 0.0851 & \pm 0.012 & {$[8.15]$} & {$[0.000202]$} & $\{0.115\}$ & 0.000528 & \pm 0.00031 \\
\hline & 4 & 0.197 & \pm 0.023 & {$[3.52]$} & {$[0.000311]$} & {$[0.177]$} & 0.000815 & \pm 0.00013 \\
\hline & 5 & 0.356 & \pm 0.051 & {$[1.95]$} & {$[0.000576]$} & {$[0.326]$} & 0.00151 & \pm 0.00024 \\
\hline & 6 & $1.35^{a}$ & & [ 0.513] & {$[0.000057]$} & {$[0.032]$} & $0.00015^{a}$ & \pm 0.00087 \\
\hline & All & & & & {$[0.00176]$} & {$[1.000]$} & 0.00461 & \pm 0.00073 \\
\hline Fast $^{22}$ & 1 & 0.0140 & \pm 0.0005 & [49.5] & {$[0.000010]$} & [0.0069] & 0.000268 & \pm 0.000029 \\
\hline $2 \mathrm{MeV}$ & 2 & 0.0317 & \pm 0.0016 & {$[21.9]$} & {$[0.000424]$} & [0.291] & 0.00111 & \pm 0.00007 \\
\hline$v=2.62^{5}$ & 3 & 0.140 & \pm 0.006 & [4.95] & {$[0.000454]$} & {$[0.311]$} & 0.00119 & \pm 0.00008 \\
\hline & 4 & 0.370 & \pm 0.022 & [ 1.87 ] & {$[0.000431]$} & {$[0.295]$} & 0.00113 & \pm 0.00012 \\
\hline & 5 & 1.20 & \pm 0.60 & {$[0.578]$} & {$[0.000105]$} & {$[0.072]$} & 0.000274 & \pm 0.000091 \\
\hline & 6 & & - & - & {$[0.000035]$} & {$[0.024]$} & $0.000092^{b}$ & $b \pm 0.000023$ \\
\hline & All & & & & {$[0.00155]$} & {$[1.000]$} & 0.00406 & \pm 0.00020 \\
\hline Fast $^{23}$ & All & & & & & & 0.004510 & \pm 0.00051 \\
\hline Reactor 23 & All & & & & & & 0.00456 & \pm 0.00051 \\
\hline
\end{tabular}

aAssumed values.

balculated. 
TABLE A-X. Plutonium-239

Values in [ ] are deduced from other values in this table.

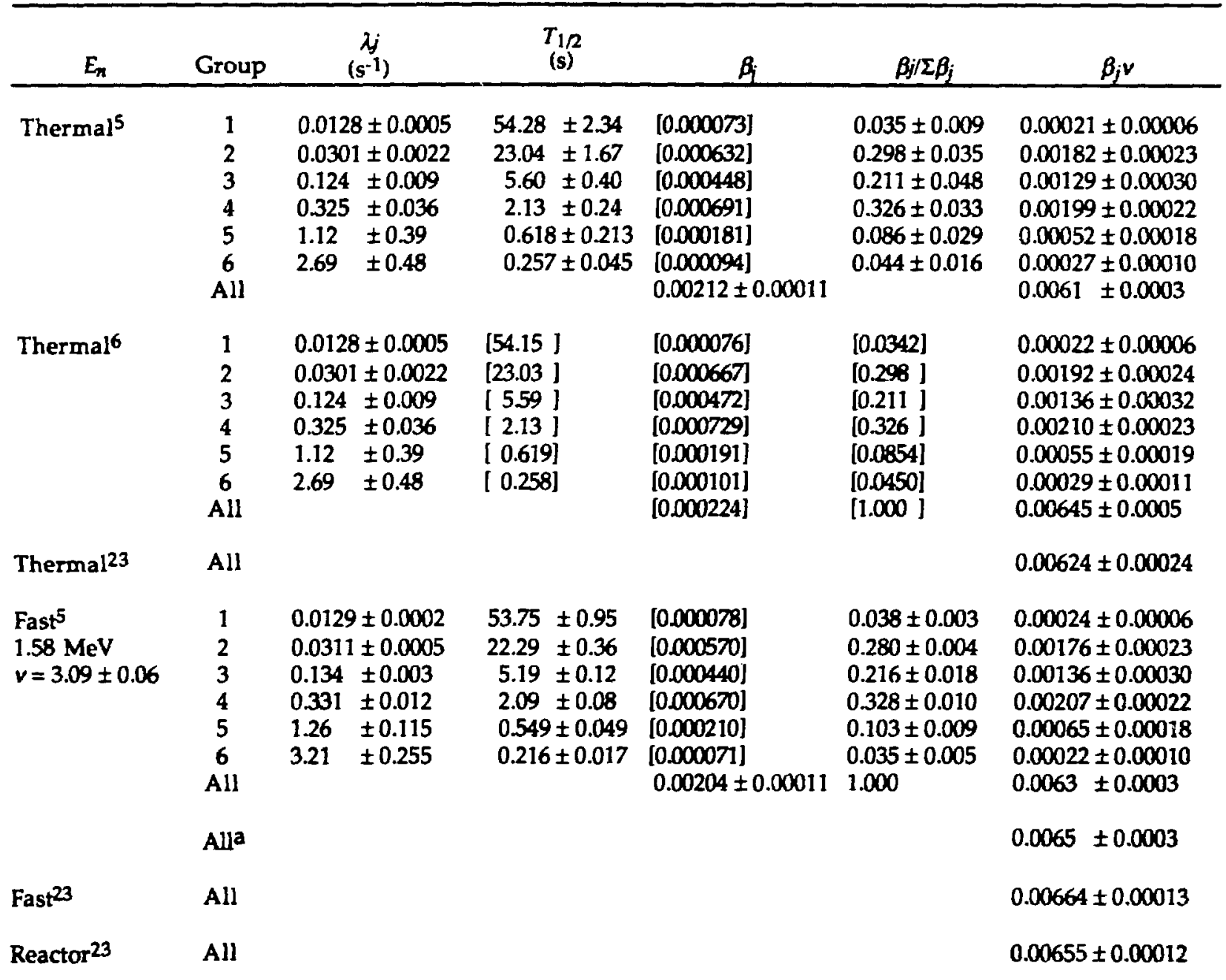

average of several laboratories' values. 
TABLE A-XI. Plutonium-240

Values in [ ] are deduced from other values in this table.

\begin{tabular}{|c|c|c|c|c|c|c|c|c|}
\hline$E_{n}$ & Group & & $\begin{array}{c}\lambda \\
\left(\mathrm{s}^{-1}\right)\end{array}$ & $\begin{array}{c}T_{1 / 2} \\
(\mathrm{~s})\end{array}$ & $B$ & $\beta j / \Sigma \beta_{j}$ & & $\beta_{j} v$ \\
\hline $\begin{array}{l}\text { Fast }^{5} \\
2.13 \mathrm{MeV} \\
v=3.3 \pm 0.2\end{array}$ & $\begin{array}{c}1 \\
2 \\
3 \\
4 \\
5 \\
6 \\
\text { All" } \\
\text { Alla }\end{array}$ & $\begin{array}{l}0.0129 \\
0.0313 \\
0.135 \\
0.333 \\
1.36 \\
4.04\end{array}$ & $\begin{array}{l} \pm 0.0004 \\
\pm 0.0005 \\
\pm 0.011 \\
\pm 0.031 \\
\pm 0.205 \\
\pm 0.782\end{array}$ & $\begin{array}{l}53.56 \pm 1.21 \\
22.14 \pm 0.38 \\
5.14 \pm 0.42 \\
2.08 \pm 0.19 \\
0.511 \pm 0.077 \\
0.172 \pm 0.033\end{array}$ & $\begin{array}{l}{[0.000085]} \\
{[0.000721]} \\
{[0.000491]} \\
{[0.000955]} \\
{[0.000361]} \\
{[0.000073]} \\
0.00266 \pm 0.00024\end{array}$ & $\begin{array}{ll}0.028 & \pm 0.003 \\
0.273 & \pm 0.004 \\
0.192 & \pm 0.053 \\
0.350 & \pm 0.020 \\
0.128 & \pm 0.018 \\
0.029 & \pm 0.006 \\
1.000 & \end{array}$ & $\begin{array}{l}0.00028 \\
0.00238 \\
0.00162 \\
0.00315 \\
0.00119 \\
0.00024 \\
0.0088 \\
0.0088\end{array}$ & $\begin{array}{l} \pm 0.00003 \\
\pm 0.00016 \\
\pm 0.00044 \\
\pm 0.00027 \\
\pm 0.00018 \\
\pm 0.00005 \\
\pm 0.0006 \\
\pm 0.0006\end{array}$ \\
\hline Fast ${ }^{6}$ & $\begin{array}{c}1 \\
2 \\
3 \\
4 \\
5 \\
6 \\
\text { All }\end{array}$ & $\begin{array}{l}0.0129 \\
0.0313 \\
0.135 \\
0.333 \\
1.36 \\
4.03\end{array}$ & $\begin{array}{l} \pm 0.0003 \\
\pm 0.0005 \\
\pm 0.11 \\
\pm 0.030 \\
\pm 0.20 \\
\pm 0.77\end{array}$ & $\begin{array}{l}{[53.73]} \\
{[22.15]} \\
{[5.13]} \\
{[2.08]} \\
{[0.510]} \\
{[0.172]}\end{array}$ & $\begin{array}{ll}{[0.0249} & ] \\
{[0.269} & ] \\
{[0.183} & ] \\
{[0.356} & ] \\
{[0.134} & ] \\
{[0.0328} & ]\end{array}$ & $\begin{array}{l}0.00022 \pm 0.00003 \\
0.00238 \pm 0.00016 \\
0.00162 \pm 0.00044 \\
0.00315 \pm 0.00027 \\
0.00119 \pm 0.00018 \\
0.00029 \pm 0.00006 \\
{[1.000]}\end{array}$ & 0.0088 & \pm 0.0006 \\
\hline Fast $^{19}$ & $\begin{array}{c}1 \\
2 \\
3 \\
4 \\
5 \\
6 \\
\text { All }\end{array}$ & $\begin{array}{l}0.0128 \\
0.0317 \\
0.115 \\
0.270 \\
0.797 \\
-\end{array}$ & $\begin{array}{l} \pm 0.0003 \\
\pm 0.0007 \\
\pm 0.005 \\
\pm 0.011 \\
\pm 0.192\end{array}$ & $\begin{array}{l}{[542]} \\
\{21.9] \\
\{6.03\} \\
\{2.57\} \\
{[0.870]} \\
{[-]}\end{array}$ & & $\begin{array}{l}{[0.0291]} \\
{[0.288]} \\
{[0.138]} \\
{[0.352]} \\
{[0.163]} \\
{[0.0285]} \\
{[1.000]}\end{array}$ & $\begin{array}{l}0.000265 \\
0.00263 \\
0.00126 \\
0.00321 \\
0.00149 \\
0.00026 b \\
0.00911\end{array}$ & $\begin{array}{l} \pm 0.000025 \\
\pm 0.00011 \\
\pm 0.00008 \\
\pm 0.00014 \\
\pm 0.00023 \\
\pm 0.00006 \\
\pm 0.00041\end{array}$ \\
\hline Fast ${ }^{23}$ & All & & & & & & 0.0096 & \pm 0.0011 \\
\hline Reactor 23 & All & & & & & & 0.0096 & \pm 0.0021 \\
\hline
\end{tabular}


TABLE A-XII. Plutonium-241

Values in [ ] are deduced from other values in this table.

\begin{tabular}{|c|c|c|c|c|c|c|c|}
\hline$E_{n}$ & Group & $\begin{array}{c}\lambda j \\
\left(s^{-1}\right)\end{array}$ & $\begin{array}{c}T_{1 / 2} \\
(\mathrm{~s})\end{array}$ & $\beta_{j}$ & $\beta j / \Sigma \beta_{j}$ & & $\beta_{j} v$ \\
\hline $\begin{array}{l}\text { Thermal }^{5} \\
v=3.14^{ \pm} 0.06\end{array}$ & $\begin{array}{c}1 \\
2 \\
3 \\
4 \\
5 \\
6 \\
\text { All }\end{array}$ & $\begin{array}{c}{[0.0128]} \\
{[0.0299]} \\
{[0.124]} \\
{[0.352]} \\
{[1.61]} \\
-\end{array}$ & $\begin{array}{l}54.0 \pm 1 \\
23.2 \pm 0.5 \\
5.6 \pm 0.6 \\
1.97 \pm 0.1 \\
0.43 \pm 0.04 \\
-\end{array}$ & $\begin{array}{l}{[0.000049]} \\
{[0.00116]} \\
{[0.000876]} \\
{[0.00197]} \\
{[0.000924]} \\
{[-]} \\
0.0049 \pm 0.0005\end{array}$ & $\begin{array}{l}{[0.0099]} \\
{[0.233]} \\
{[0.176]} \\
{[0.396]} \\
{[0.185]} \\
{[-]} \\
{[1.000]}\end{array}$ & $\begin{array}{l}0.000154 \\
0.00365 \\
0.00275 \\
0.00620 \\
0.00290 \\
0.0154\end{array}$ & $\begin{array}{l}4 \pm 0.00004 \\
\pm 0.0001 \\
\pm 0.0004 \\
\pm 0.0008 \\
\pm 0.0003 \\
\pm 0.0015\end{array}$ \\
\hline Thermal $^{6}$ & $\begin{array}{c}1 \\
2 \\
3 \\
4 \\
5 \\
6 \\
\text { All }\end{array}$ & $\begin{array}{l}0.0128 \pm 0.0002 \\
0.0299 \pm 0.0006 \\
0.124 \pm 0.013 \\
0.352 \pm 0.018 \\
1.61 \pm 0.15 \\
3.47 \pm 1.70\end{array}$ & 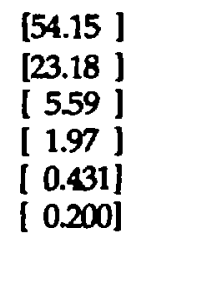 & & $\begin{array}{l}{[0.00995]} \\
{[0.228]} \\
{[0.178]} \\
{[0.388]} \\
{[0.181]} \\
{[0.0159]} \\
{[1.000]}\end{array}$ & $\begin{array}{l}0.000156 \\
0.00357 \\
0.00279 \\
0.00608 \\
0.00284 \\
0.00025 \\
0.0157\end{array}$ & $\begin{array}{l}6 \pm 0.000047 \\
\pm 0.00009 \\
\pm 0.00039 \\
\pm 0.00078 \\
\pm 0.00030 \\
\pm 0.00008 \\
\pm 0.0015\end{array}$ \\
\hline Thermal 23 & All & & & & & 0.0156 & \pm 0.0016 \\
\hline Fast 19 & $\begin{array}{c}1 \\
2 \\
3 \\
4 \\
5 \\
6 \\
\text { All }\end{array}$ & $\begin{array}{l}0.0128 \pm 0.0002 \\
0.0317 \pm 0.0009 \\
0.137 \pm 0.009 \\
0.355 \pm 0.033 \\
1.26 \pm 0.38 \\
\quad-\end{array}$ & 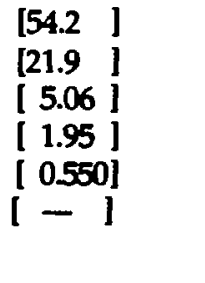 & & $\begin{array}{l}{[0.0172]} \\
{[0.231]} \\
{[0.186]} \\
{[0.367]} \\
{[0.172]} \\
{[0.0274]} \\
{[1.000]}\end{array}$ & $\begin{array}{l}0.000276 \\
0.00370 \\
0.00298 \\
0.00589 \\
0.00276 \\
0.00044^{a} \\
0.0160\end{array}$ & $\begin{array}{l}6 \pm 0.000012 \\
\pm 0.00011 \\
\pm 0.00036 \\
\pm 0.00051 \\
\pm 0.00055 \\
\pm 0.00011 \\
\pm 0.0009\end{array}$ \\
\hline Fast $^{23}$ & All & & & & & 0.0163 & \pm 0.0016 \\
\hline Reactor 23 & All & & & & & 0.0160 & \pm 0.0016 \\
\hline
\end{tabular}


TABLE A-XIII. Plutonium-242

Values in I] are deduced from other values in this table.

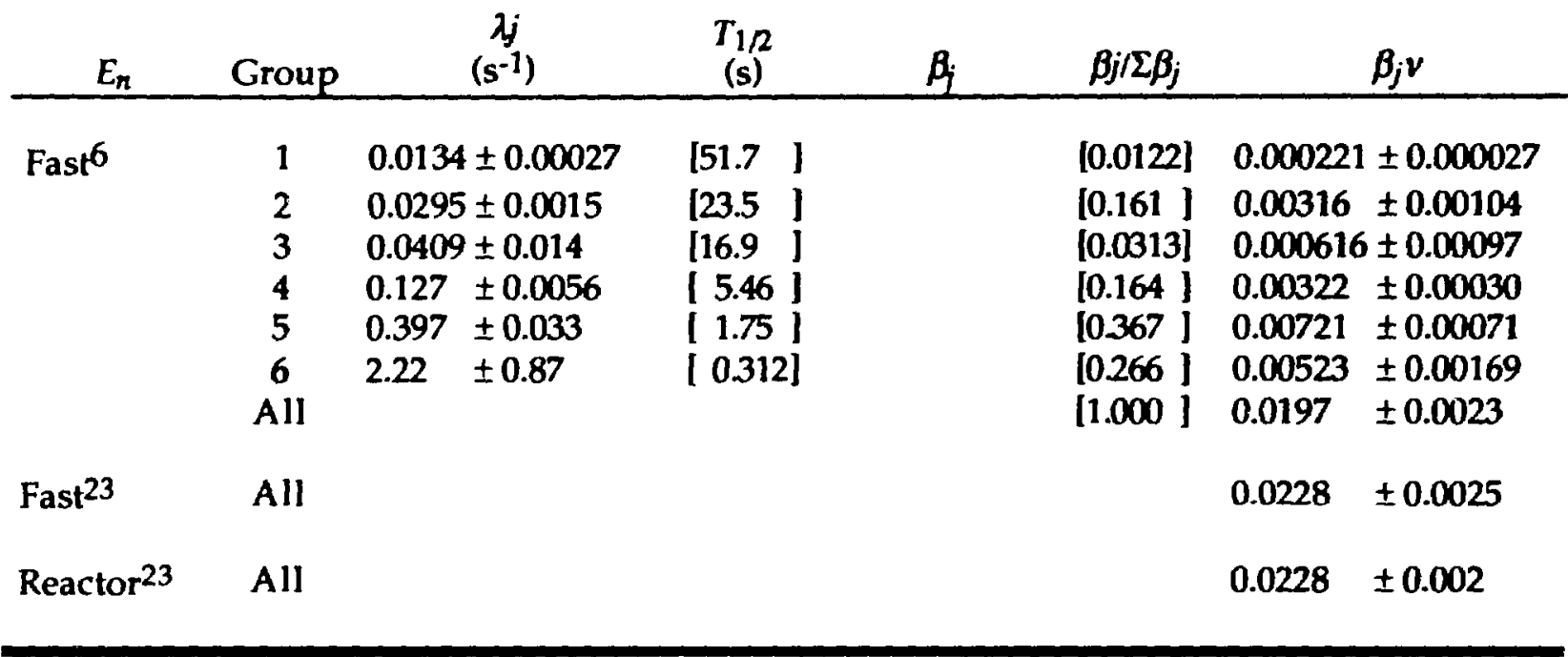

TABLE A-XIV. Americium-241

Values in [ ] are deduced from other values in this table.

\begin{tabular}{|c|c|c|c|c|c|c|c|c|}
\hline$E_{n}$ & Group & & $\begin{array}{c}\lambda_{j} \\
(s-1)\end{array}$ & $\begin{array}{l}\mathrm{T}_{1 / 2} \\
(\mathrm{~s})\end{array}$ & $\beta j$ & $\beta_{i} \sqrt{\Sigma} \beta_{i}$ & & $3_{j} V$ \\
\hline \multirow[t]{7}{*}{ Thermal 6} & 1 & 0.0127 & $71 \pm 0.00003$ & [54.54] & {$[0.000060]$} & [0.0364] & 0.000185 & \pm 0.000022 \\
\hline & 2 & 0.0298 & $35 \pm 0.00004$ & [23.22 ] & {$[0.000472]$} & [0.287] & 0.00146 & \pm 0.00018 \\
\hline & 3 & 0.152 & \pm 0.003 & {$[456]$} & {$[0.000498]$} & {$[0.303]$} & 0.00154 & \pm 0.00019 \\
\hline & 4 & 0.446 & \pm 0.022 & {$[1.55]$} & {$[0.000498]$} & {$[0.303]$} & 0.00154 & \pm 0.00020 \\
\hline & 5 & 2.63 & \pm 2.11 & {$[0.264]$} & {$[0.000117]$} & {$[0.0708]$} & 0.00036 & \pm 0.00048 \\
\hline & 6 & & -- & - & {$[-1$} & {$[-]$} & & - \\
\hline & All & & & & {$[0.001645]$} & [1.000] & 0.00509 & \pm 0.00060 \\
\hline \multirow[t]{7}{*}{ Fast 22} & 1 & 0.0122 & \pm 0.0002 & {$[56.8]$} & & [0.0488] & 0.000192 & \pm 0.000021 \\
\hline & 2 & 0.0310 & \pm 0.0011 & {$[22.4]$} & & {$[0.295]$} & 0.00116 & \pm 0.00009 \\
\hline & 3 & 0.126 & \pm 0.011 & {$[5.50]$} & & {$[0.166]$} & 0.000655 & \pm 0.000137 \\
\hline & 4 & 0.262 & \pm 0.015 & {$[2.65]$} & & {$[0.343]$} & 0.00135 & \pm 0.00009 \\
\hline & 5 & 0.85 & \pm 0.30 & {$[0.82]$} & & {$[0.125]$} & 0.000493 & \pm 0.000098 \\
\hline & 6 & & - & $1-1$ & & {$[0.0221]$} & $0.000087^{\circ}$ & \pm 0.000022 \\
\hline & All & & & & & {$[1.000]$} & 0.00394 & \pm 0.00024 \\
\hline
\end{tabular}

acalculated. 


\section{REFERENCES}

1. D. R. Rogers, Handbook of Nuclear Safeguards Measurement Methods, NUREG/CR-2078, MLM-2855, United States Nuclear Regulatory Commission, September 1983, pp. 629-648.

2. T. Gozani, Active Nondestructive Assay of Nuclear Materials, Principles and Applications, NUREG/CR-0602, SAI-MLM-2585, United States Nuclear Regulatory Commission, January 1981, pp. 286-297.

3. S. E. Binney and R. I. Scherpelz, "A Review of the Delayed Fission Neutron Technique," Nucl. Instrum. Methods 154, 413-431 (1978).

4. T. Crane, "Measurement of Uranium and Plutonium in Solid Waste by Passive Photon or Neutron Counting and Isotopic Neutron Source Interrogation," Los Alamos Scientific Laboratory report LA-8294-MS (March 1980).

5. G. R. Keepin, Physics of Nuclear Kinetics (Addison-Wesley Publishing Company, Inc., Reading, Massachusetts, 1965).

6. R. W. Waldo, R. A. Karam, and R. A. Meyer, "Delayed Neutron Yields: Time Dependent Measurements and a Predictive Model," Phys. Rev. C, 23(3), 1113-1127 (March 1981).

7. A. C. Wahl, "Nuclear-Charge Distribution and Delayed-Neutron Yields for Thermal-Neutron-Induced Fission of ${ }^{235} \mathrm{U},{ }^{233} \mathrm{U}$, and ${ }^{239} \mathrm{Pu}$ and for Spontaneous Fission of ${ }_{1}^{252} \mathrm{Cf}, "$ Atomic Data and Nuclear Data Tables 39(1), May 1988, pp. 1-156.

8. M. C. Brady and T. R. England, "Delayed Neutron Data and Group Parameters for 43 Fissioning Systems," Nucl. Sci. Eng. 103, 129-149 (October 1989).

9. G. Tessler, B. R. Beaudoin, W. J. Beggs, L. B. Freeman, A. C. Kahler, and W. C. Schick, "A Gauge for Nondestructive Assay of Irradiated Fuel Rods," Nucl. Technol. 82, 275-289 (September 1988).

10. L. Tomlinson and M. H. Hurdus, "Search for Long-Lived Delayed Neutron Groups: Photofission Caused by Fission-Product Gamma-Rays," Physics and Chemistry of Fission, Proceedings of the Second IAEA Symposium on the Physics and Chemistry of Fission, Vienna, 28 July - 1 August 1969 (International Atomic Energy Agency, Vienna, 1969), pp. 605-620.

11. S. Amiel and Jacob Gilat, "Reactions $\mathrm{O}^{17}(\mathrm{n}, \mathrm{p}) \mathrm{N}^{17}$ and $\mathrm{O}^{18}(\mathrm{n}, \mathrm{d}) \mathrm{N}^{17}$ with Reactor Neutrons," Nucl. Sci. Eng. 18, 105-109 (1964).

12. H. O. Menlove, R. H. Augustson, and C. N. Henry, "Cross Section for the Delayed Neutron Yield from the ${ }^{17} \mathrm{O}(\mathrm{n}, \mathrm{p})^{17} \mathrm{~N}$ Reaction at $14.1 \mathrm{MeV}, " \mathrm{Nucl}$. Sci. Eng. 40, 136-137 (1970).

13. G. R. Keepin, H. O. Menlove, M. M. Thorpe, R. H. Augustson, C. N. Henry, D. B. Smith, and T. D. Reilly, "Application Areas and Results of Non-Destructive Assay Measurements," Safeguards Techniques, Proceedings of a Symposium, Karlsruhe, 6-10 July 1970 (International Atomic Energy Agency, Vienna, 1970), Vol. II, pp. 79-112. 
14. H. E. Hootman, "Estimation of ${ }^{252} \mathrm{Cf}$ Shielding Requirements," Savannah River Laboratory report DP-1232, Physics, TID-4500 (April 1970).

15. George W. Eccleston, "Analytical Applications for Delayed Neutrons," Los Alamos National Laboratory document LA-UR-83-3058 (1983).

16. G. W. Eccleston, "Nuclear Safeguards Research and Development, Program Status Report, July-September 1979," Los Alamos National Laboratory report LA-8125-PR (January 1980), pp. 7-9.

17. K. W. MacMurdo and W. W. Bowman, "Assay of Fissile Materials by a Cyclic Method of Neutron Activation and Delayed Neutron Counting," Nucl. Instrum. Methods 141, 299-306 (1977).

18. Y. Matsuda, T. Tamura, and T. Murata, "Non-Destructive Active Assay of Uranium-235 and Plutonium-239 in Nuclear Reactor Fuel," Nuclear Safeguards Technology 1978, Proceedings of a Symposium, Vienna, 2-6 October 1978 (International Atomic Energy Agency, Vienna, 1979), Vol. II, pp. 113-124.

19. T. W. Crane, E. L. Adams, C. M. Schneider, and N. Ensslin, 'In-Cabinet Shuffler Start-Up and Operating Procedures Manual," Los Alamos National Laboratory document LA-UR-87-2546, July 28, 1987, Appendix A, Signal Calculation and Calibration, Cycle Correction Factor, p. 57.

20. T. W. Crane, "Liquid-Sample Shuffler for Gamma Active Solutions," Los Alamos National Laboratory report LA-10925-MS (February 1987), p. 9.

21. T. W. Crane, "Detectability Limits and Precision for Shufflers," Los Alamos National Laboratory report LA-10158-MS (August 1984).

22. G. Benedetti, A. Cesana, V. Sangiust, and M. Terrani, "Delayed Neutron Yields from Fissions of Uranium-233, Neptunium-237, Plutonium-238, -240, -241, and Americium-241," Nucl. Sci. Eng. 80, 379-387 (1982).

23. R. J. Tuttle, "Delayed-Neutron Data for Reactor-Physics Analysis," Nucl. Sci. Eng. 56, 37-71 (1975).

24. H. O. Menlove and T. W. Crane, "4 ${ }^{252} \mathrm{Cf}$ Based Nondestructive Assay System for Fissile Material," Nucl. Instrum. Methods 152, 549-557 (1978).

25. S. -T. Hsue, T. E. Sampson, J. L. Parker, S. S. Johnson, and D. F. Bowersox, "Plutonium Isotopic Composition by Gamma-Ray Spectroscopy," Los Alamos National Laboratory report LA-8603-MS (November 1980).

26. T. E. Sampson, E. L. Sandford, D. F. Bowersox, S. S. Johnson, S. -T. Hsue, J. L. Parker, K. Kroncke, and G. Walton, "In-Plant Experience with Automated Gamma-Ray Spectroscopy Systems for Plutonium Isotopic Composition Measurements," Los Alamos National Laboratory report LA-9789-MS (August 1983).

27. S. D. Clement and R. H. Augustson, "Plutonium Isotopic Analysis of Fighly Enriched Mixed Oxides," Los Alamos National Laboratory report LA-10811-MS (August 1986). 
28. J. E. Foley and G. E. Bosler, "High-Level Neutron Coincidence Counter (HLNCC) Implementation: Assay of the Plutonium Content of Mixed-Oxide Fuel Assemblies," Los Alamos National Laboratory report LA-9318-MS (April 1982).

29. H. O. Menlove, R. Abedin-Zadeh, and R. Zhu, "The Analyses of Neutron Coincidence Data to Verify Both Spontaneous-Fission and Fissionable Isotopes," Los Alamos National Laboratory report LA-11639-MS (August 1989).

30. T. W. Packer, M. T. Swinhoe, and D. B. Syme, "Non-Destructive Assay of the Fissile Content of Radioactive Waste Using a Californium Shuffler," 11th Annual Symposium on Safeguards and Nuclear Material Management, (Luxembourg, Commission of European Communities, 1989), ESARDA report EUR 12193 EN.

31. N. Baron, "A Correction for Variable Moderation and Multiplication Effects Associated with Thermal Neutron Coincidence Counting," Nuclear Safeguards Technology 1978, Proceedings of a Symposium on Nuclear Material Safeguards, Vienna, October 2-6, 1978 (International Atomic Energy Agency, Vienna, 1979), pp. 161-177. 